\title{
Modelagem Bayesiana para dados de sobrevivência bivariados através de cópulas
}

\author{
José Santos Romeo Núñez
}

TESE APRESENTADA

$\mathrm{AO}$

INSTITUTO DE MATEMÁTICA E ESTATÍSTICA

$\mathrm{DA}$

UNIVERSIDADE DE SÃO PAULO

PARA OBTENÇÃO DO GRAU DE DOUTOR

EM

ESTATÍSTICA

\author{
Área de concentração: Estatística \\ Orientador: Prof. Dr. Antonio Carlos Pedroso de Lima \\ Co-Orientador: Prof. Dr. Nelson Ithiro Tanaka
}
Durante o curso de Doutorado e elaboração deste trabalho
o autor recebeu apoio financeiro do $\mathrm{CNPq}$

- São Paulo. agosto de 2005 - 


\title{
Modelagem Bayesiana para dados de sobrevivência bivariados através de cópulas
}

\author{
Este exemplar corresponde à redação \\ final da tese corrigida e defendida \\ por José Santos Romeo Núñez e \\ aprovada pela comissão julgadora.
}

São Paulo, agosto de 2005.

Banca examinadora:

- Prof. Dr. Antonio Carlos Pedroso de Lima (Orientador) - IME-USP

- Prof. Dr. Nelson Ithiro Tanaka (Co-Orientador) - IME-USP

- Prof. Dr. Pranab K. Sen - University of North Carolina

- Prof. Dr. Josemar Rodrigues - DEs-UFSCar

- Prof. Dra. Verónica González-López - IMECC-LNICAMIP 
A Claudia mi amor.

A mis niños Fredo. Angie y Benja.

A mi Familia y Amigos. 


\section{AGRADECIMENTOS}

Aos meus orientadores, Prof. Antonio Carlos e Prof. Nelson, pela dedicação, confiança, paciência e incentivo dado durante estes anos.

Aos Profs. Márcia, Carlinhos, Heleno, Sérgio e Nikolai, que contribuiram para a minha formação.

Ao Dr. Sen pelas valiosas sugestões e revisão deste trabalho.

Ao Prof. Victor Hugo Salinas, por tudo que fez por mim, e a SalinasTorres (1992) pela inspiração.

Ao CNPq pelo apoio financeiro.

A todos meus amigos e colegas do IME. Em especial a: Adriano, Murilo, Daniel, Cacá, Alfredo, Casado, Ângela, Gisela, Jorge, Felipe, Thales, Cristian, Edijane e Camilo.

Ao pessoal da Atlética do IME e aos meus amigos do Handebol pela diversão. 


\title{
RESUMO
}

Este trabalho apresenta uma abordagem Bayesiana paramétrica para o estudo de dados bivariados em Análise de Sobrevivência. Considerando cópulas Arquimedianas para modelar a estrutura de dependência entre os tempos de falha, procedimentos Bayesianos de estimação para os parâmetros de interesse são propostos e ilustrados através de duas aplicações. Utilizando a relação funcional entre cópulas e as funções de sobrevivência marginal, o método delta para funcionais é aplicado e propriedades assintóticas do estimador da função de sobrevivência bivariada são estudadas.

\begin{abstract}
This work presents a parametric Bayesian approach to modeling bivariate data in Survival Analysis. Considering Arquimedean copula to model the dependence structure among failure times, Bayesian estimation procedures for the parameters of interest are proposed and illustrated through two applications. Using the functional relationship between the copula and the marginal survival functions, the functional delta method is applied and large sample properties of the bivariate survival function estimator are studied.
\end{abstract}




\section{Sumário}

1 Introdução Geral 1

1.1 Introdução . . . . . . . . . . . . . . . . . . . 1

1.2 Cópulas . . . . . . . . . . . . . . . . . 5

1.3 Cópulas Arquimedianas $\ldots \ldots \ldots \ldots$

1.4 Tau de Kendall . . . . . . . . . . . . . . . . . . . . . . 11

1.5 Objetivos da Tese . . . . . . . . . . . . . . . . . . 13

2 Modelagem e Estimação 14

2.1 Introdução . . . . . . . . . . . . . . . . . . . 14

2.2 Modelos de Fragilidade e Cópulas Arquimedianas . . . . . 14

2.2 .1 Família de Clayton . . . . . . . . . . . . . . 18

2.2.2 Fragilidade Estável Positiva . . . . . . . . . . . . . 19

2.2.3 Família de Frank . . . . . . . . . . . . . . . 20

2.3 Estimação Bayesiana . . . . . . . . . . . . . . . . . . . 21

2.4 Aplicações . . . . . . . . . . . . . . . . . . . . . . . 25

2.4.1 Pacientes com Retinopatia Diabética . . . . . . . 2. 25

2.4.2 Duração da Remissão de Pacientes com Leucemia . . . 43 
3 Teorema Central do Limite Funcional para Cópulas em Análise de Sobrevivência $\quad 49$

3.1 Introdução . . . . . . . . . . . . . . . . . . . . . . . . . . . . 49

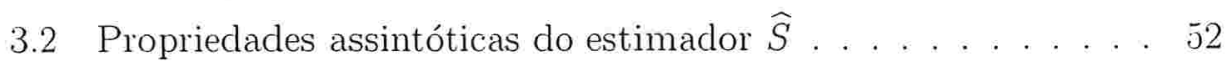

3.2 .1 Consistência forte de $\dot{C}_{\alpha_{n}^{*}} \ldots \ldots \ldots \ldots \ldots$

3.2.2 Consistência forte de $C_{o}\left(\widehat{S}_{1 n}, \widehat{S}_{2 n}\right) \ldots \ldots \ldots$

3.2.3 Convergência fraca de $C_{o}\left(\widehat{S}_{1 n}, \widehat{S}_{2 n}\right) \ldots \ldots 62$

3.2 .4 Consistência forte de $\widehat{S} \ldots \ldots \ldots \ldots$

3.2 .5 Convergência fraca de $\widehat{S} \ldots \ldots \ldots$. . . . . . 71

3.2.6 Prova Proposições 3.2.1, 3.2 .2 e parte (i) Teorema $3.2 .3(\mathrm{a}) \ldots \ldots \ldots \ldots$

4 Considerações finais e perspectivas de trabalhos futuros 82

A Método Delta para Funcionais $\quad 85$

$\begin{array}{ll}\text { B Alguns conceitos e definições } & 91\end{array}$

Referências Bibliográficas $\quad 94$ 


\section{Capítulo 1}

\section{Introdução Geral}

\subsection{Introdução}

Quando lidamos com dados de sobrevivência bivariados, a suposição de independência entre tempos de falha pode não ser sustentável de forma que as técnicas mais difundidas podem ser inadequadas. Um exemplo bastante discutido, considerado por Huster, Brookmeyer e Self (1989), baseia-se no estudo The Diabetic Retinopathy Study (1976), que considera pacientes diabéticos submetidos a terapia de laser em um dos olhos, escolhido aleatoriamente. O evento de interesse principal é a perda da acuidade visual. É natural assumir que existe uma associação entre os tempos de falha em um determinado paciente, como também é de interesse considerar esta associação quando se estuda o efeito de covariáveis nas respostas.

Vários enfoques têm sido considerados para lidar com dados de sobrevivência multivariados. Os mais populares são os modelos de fragilidade em que um ou mais efeitos aleatórios são incluídos na função de taxa de falha, para levar em conta a dependência entre as obserrações. Neste caso, os tempos 
marginais são considerados condicionalmente independentes dada, a variável de fragilidade. Detalhes iniciais no desenvolvimento de tais modelos podem ser vistos em Clayton (1978). Hougaard (2000) apresenta um completo desenvolvimento destes modelos e Therneau e Grambsch (2000) discutem a implementação computacional destes métodos.

Como uma alternativa a esse abordagem podemos modelar as distribuições marginais separadamente, em um primeiro passo, e então asumir uma estrutura de associação usando uma família de cópulas (modelos de abordagem marginal).

Cópulas fornecem um meio de relacionar funções de distribuição multivariadas com suas funções de distribuição marginais.

Seja $X_{j}$ uma variável aleatória com função de distribuição marginal contínua $F_{j}, j=1, \ldots, p$. A dependência entre as variáveis aleatórias $X_{1}, \ldots, X_{p}$ é completamente descrita por sua função de distribuição conjunta

$$
F\left(x_{1}, \ldots, x_{p}\right)=\operatorname{Pr}\left\{X_{1} \leq x_{1}, \ldots, X_{p} \leq x_{p}\right\}
$$

Considerando que $F_{j}\left(X_{j}\right) \sim U(0,1)$, a distribuição conjunta $C_{\alpha}$ de $\left(F_{1}\left(X_{1}\right), \ldots, F_{p}\left(X_{p}\right)\right)$ é chamada de cópula do vetor aleatório $\left(X_{1}, \ldots, X_{p}\right)$, indexada por um parâmetro de dependência $\alpha \in \mathcal{A}$. Assim, considerando $U_{j}=F_{j}\left(X_{j}\right)$,

$$
\begin{aligned}
F\left(x_{1}, \ldots, x_{p}\right) & =\operatorname{Pr}\left\{U_{1} \leq F_{1}\left(x_{1}\right), \ldots, U_{p} \leq F_{p}\left(x_{p}\right)\right\} \\
& =C_{\alpha}\left(F_{1}\left(x_{1}\right) \ldots, F_{p}\left(x_{p}\right)\right),
\end{aligned}
$$

que é uma função de distribuição multivariada, definida no cubo $[0,1]^{p}$. com marginais uniformemente distribuídas. 
Da expressão anterior, tem-se que a função de densidade conjunta pode ser escrita como

$$
f\left(x_{1}, \ldots, x_{p}\right)=c_{\alpha}\left(F_{1}\left(x_{1}\right), \ldots, F_{p}\left(x_{p}\right)\right) \prod_{j=1}^{p} f_{j}\left(x_{j}\right),
$$

em que $c_{\alpha}$ é a função de densidade associada à cópula $C_{\alpha}$. Note que as marginais e a estrutura de dependência podem ser olhadas separadamente e esta estrutura de dependência pode ser representada por uma cópula.

Veja Schweizer e Sklar (1983), Joe (1997) ou Nelsen (1999) para um estudo mais geral sobre cópulas.

O procedimento clássico de estimação do parâmetro de dependência $\alpha \in \mathcal{A}$, envolve duas etapas. Em uma primeira etapa são estimadas as distribuições marginais sem levar em conta a dependência entre as variáveis, e em uma segunda etapa o estimador do parâmetro de dependência $\alpha$ é obtido, maximizando uma pseudo verossimilhança em que as marginais são consideradas fixas e substituídas por seus estimadores obtidos na primeira etapa da estimação. Conforme Joe (1997), este tipo de estimação paramétrica, baseada nas funções de verosimilhança marginais, é chamado de método de funções de inferência para marginais (IFM methods, veja McLeish e Small, 1988), em que as funções de inferência ou estimação são funções score de funções de verossimilhança das distribuições marginais. Um procedimento similar, mas considerando estimação não paramétrica na primeira etapa de estimação, é dado em Genest, Ghoudi e Rivest (1995). Eles consideram como estimadores das distribuições marginais as correspondentes funções de distribuição empírica.

Embora cópulas se apliquem em muitas áreas, em Análise de Sobrevivència 
são úteis quando o interesse principal é modelar a associação entre tempos bivariados pois, como foi visto, o efeito da estrutura de dependência pode ser separado das distribuições marginais.

Se $S_{j}$ representa a função de sobrevivência marginal de $X_{j}$, tem-se que $S_{j}\left(X_{j}\right) \sim U(0,1), j=1, \ldots, p$. Assim, para funções de sobrevivência multivariadas também existe uma representação baseada em cópula, isto é, considerando $U_{j}=S_{j}\left(X_{j}\right)$, tem-se que a função de sobrevivência conjunta pode ser escrita como

$$
\begin{aligned}
S\left(x_{1}, \ldots, x_{p}\right) & =\operatorname{Pr}\left\{F_{1}\left(X_{1}\right)>F_{1}\left(x_{1}\right), \ldots, F_{p}\left(X_{p}\right)>F_{p}\left(x_{p}\right)\right\} \\
& =\operatorname{Pr}\left\{U_{1} \leq S_{1}\left(x_{1}\right), \ldots, U_{p} \leq S_{p}\left(x_{p}\right)\right\} \\
& =C_{\alpha}\left(S_{1}\left(x_{1}\right), \ldots, S_{p}\left(x_{p}\right)\right) .
\end{aligned}
$$

Em Análise de Sobrevivência, modelos baseados em cópulas são considerados, por exemplo, em Hougaard (1989), Oakes (1989), Shih e Louis (1995) e Gustafson, Aeschliman e Levy (2003). Dado que em um modelo de cópula as distribuições marginais não dependem da escolha da estrutura de dependência e o procedimento de estimação pode ser executado em duas etapas, Hougaard (1989) considera em um primeiro passo, a estimação de cada marginal usando o estimator de Nelson para a função de taxa de falha acumulada, ignorando a dependência entre tempos de falha. Em um segundo passo um modelo de cópula é adotado e o parâmetro de dependência, $\alpha \in \mathcal{A}$, é estimado considerando as distribuições marginais fixas (estimadas no primeiro passo). Shih e Louis (1995) consideram o procedimento de duas etapas para modelos paramétricos e semi-paramétricos, fazendo uma estimação paramétrica e usando o estimador de Kaplan-Meier para as distribuiçōes marginais. respectivamente. Como em Hougaard (1989), 
eles assumem independência neste passo e incluem o parâmetro de dependência na segunda etapa. Gustafson, Aeschliman e Levy (2003) aplicam amostragem de importância (veja Tanner, 1996, Capítulo 5) para ajustar modelos de sobrevivência Bayesiana. Eles usam um modelo de cópula para modelar dados bivariados considerando que as distribuições marginais dependem de (logaritmo) funções de taxa de falha constantes por partes (piecewise) e consideram a estrutura de dependência baseada na cópula Normal. Por conveniência computacional, os autores empregam uma distribuição a priori Normal multivariada para o logaritmo da função de taxa de falha e usam uma aproximação log-normal para a distribuição log-gama.

Nas seções seguintes deste capítulo são apresentadas propriedades da metodologia de cópulas, uma classe de cópulas relacionadas aos modelos de fragilidade chamadas cópulas Arquimedianas, e ligações com o coeficiente tau de Kendall. O capítulo finaliza com a apresentação dos objetivos específicos deste trabalho.

\subsection{Cópulas}

Como foi dito na seção anterior, cópulas são funções que ligam funções de distribuição multivariadas com suas funções de distribuição marginais. Alternativamente, cópulas são funções de distribuição multivariadas cujas marginais são uniformes no intervalo [0, 1]. Citando Nelsen (1999): a palavra cópula foi usada pela primeira vez em um contexto matemático-estatístico em Sklar (19.59) no teorema que agora leva seu nome. que descreve as funções 
que unem conjuntamente funções de distribuições unidimensionais para formar funções de distribuição multivariada (ver Teorema 1.2 .1 a seguir).

No que segue, apresentamos as principais características de cópulas com base nos trabalhos de Embrechts et al. (2002) e Embrechts et al. (2003). Detalhes podem ser consultados em Schweizer e Sklar (1983) e, principalmente, em Joe (1997) e Nelsen (1999). Owzar e Sen (2003) apresenta também uma excelente revisão dos conceitos de cópulas, além de mostrar aplicações na área de bioestatística.

O seguinte teorema é conhecido como o Teorema de Sklar e é um importante resultado da área.

\section{Teorema 1.2.1}

Seja $F$ uma função de distribuição $p$-dimensional com marginais $F_{1}, \ldots, F_{p}$. Então existe uma cópula $C_{\alpha}$ tal que para todo $\left(x_{1}, \ldots, x_{p}\right)$ em $\mathbb{R}^{p}$,

$$
F\left(x_{1}, \ldots, x_{p}\right)=C_{\alpha}\left(F_{1}\left(x_{1}\right), \ldots, F_{p}\left(x_{p}\right)\right) .
$$

Se $F_{1}, \ldots, F_{p}$ são todas contínuas então $C_{\alpha}$ é única; caso contrário $C_{\alpha}$ é determinada unicamente na imagem $F_{1} \times \cdots \times$ imagem $F_{p}$. Inversamente, se $C_{\alpha}$ é uma cópula e $F_{1}, \ldots, F_{p}$ são funções de distribuição, então a função $F$ definida acima é uma função de distribuição $p$-dimensional com marginais $F_{1}, \ldots, F_{p}$.

Para a prova do teorema ver Sklar (1996) ou Nelsen (1999). A seguir apresenta-se um exemplo de cópula. 
Exemplo 1 (Joe, 1997, página 13).

Para $\alpha>0$, e $\left(x_{1}, x_{2}\right) \in \mathbb{R}^{2}$, considere a distribuição bivariada,

$$
F\left(x_{1}, x_{2}\right)=\exp \left\{-\left[e^{-x_{1}}+e^{-x_{2}}-\left(e^{-\alpha x_{1}}+e^{-\alpha x_{2}}\right)^{-1 / \alpha}\right]\right\} .
$$

As distribuições marginais são $F_{j}\left(x_{j}\right)=\exp \left(-e^{-x_{j}}\right), j=1,2$.

Fazendo $u_{j}=F_{j}\left(x_{j}\right)$, isto é, $x_{j}=-\ln \left(-\ln u_{j}\right), j=1,2$, obtém-se a cópula

$$
C_{\alpha}\left(u_{1}, u_{2}\right)=u_{1} u_{2} \exp \left\{\left[\left(-\ln u_{1}\right)^{-\alpha}+\left(-\ln u_{2}\right)^{-\alpha}\right]^{-1 / \alpha}\right\} .
$$

Tomando-se funções de sobrevivência marginais exponenciais, i.e., $S_{j}\left(t_{j}\right)=$ $e^{-t_{j}}, j=1,2$, tem-se que

$$
\begin{aligned}
S\left(t_{1}, t_{2}\right) & =C_{\alpha}\left(S_{1}\left(t_{1}\right), S_{2}\left(t_{2}\right)\right) \\
& =\exp \left\{-t_{1}-t_{2}+\left[t_{1}^{-\alpha}+t_{2}^{-\alpha}\right]^{-1 / \alpha}\right\} .
\end{aligned}
$$

Note que se $\alpha \rightarrow 0, \dot{S}\left(t_{1}, t_{2}\right)=e^{-t_{1}} \cdot e^{-t_{2}}$, ou seja, tem-se independência entre as variáveis.

Uma propriedade importante de cópulas é que para o vetor $\left(X_{1}, \ldots, X_{p}\right)$ de variáveis aleatórias contínuas com cópula $C_{\alpha}, X_{1}, \ldots, X_{p}$ são independentes se e somente se

$$
C_{\alpha}\left(u_{1}, \ldots, u_{p}\right)=\prod_{j=1}^{p} u_{j} .
$$

U'ma outra propriedade importante da representação de cópulas é que a estrutura de dependência é invariante a transformações contínuas e crescentes das 
marginais, ou seja, se $\left(X_{1}, \ldots, X_{p}\right)$ tem cópula $C_{\alpha}$ e $Y_{1}, \ldots, Y_{p}$ são funções contínuas e crescentes, então $\left(Y\left(X_{1}\right), \ldots, Y\left(X_{p}\right)\right)$ também tem cópula $C_{\alpha}$, isto é,

$$
\begin{aligned}
C_{\alpha}\left(F_{Y_{1}\left(X_{1}\right)}\left(x_{1}\right), \ldots, F_{Y_{p}\left(X_{p}\right)}\left(x_{p}\right)\right)=\operatorname{Pr}\left\{U_{1} \leq F_{Y_{1}\left(X_{1}\right)}\left(x_{1}\right), \ldots, U_{p} \leq F_{Y_{p}\left(X_{p}\right)}\left(x_{p}\right)\right\} \\
=\operatorname{Pr}\left\{F_{Y_{1}\left(X_{1}\right)}^{-1}\left(U_{1}\right) \leq x_{1}, \ldots, F_{Y_{p}\left(X_{p}\right)}^{-1}\left(U_{p}\right) \leq x_{p}\right\} \\
=\operatorname{Pr}\left\{Y_{1} \circ F_{1}^{-1}\left(U_{1}\right) \leq x_{1}, \ldots, Y_{p} \circ F_{p}^{-1}\left(U_{p}\right) \leq x_{p}\right\} \\
=\operatorname{Pr}\left\{Y_{1}\left(X_{1}\right) \leq x_{1}, \ldots, Y_{p}\left(X_{p}\right) \leq x_{p}\right\} \\
=\operatorname{Pr}\left\{F_{1}\left(Y_{1}\left(X_{1}\right)\right) \leq F_{1}\left(x_{1}\right), \ldots, F_{p}\left(Y_{p}\left(X_{p}\right)\right) \leq F_{p}\left(x_{p}\right)\right\} \\
=C_{\alpha}\left(F_{1}\left(x_{1}\right), \ldots, F_{p}\left(x_{p}\right)\right) .
\end{aligned}
$$

A seguir apresentamos a versão dos limites de Fréchet como cópulas.

Notando que $0 \leq \operatorname{Pr}\left\{X_{1} \leq x_{1}, \dot{X_{2}} \leq x_{2}\right\} \leq \operatorname{Pr}\left\{X_{j} \leq x_{j}\right\}, j=1,2$, e que

$$
F\left(x_{1}, x_{2}\right)=S\left(x_{1}, x_{2}\right)+F_{1}\left(x_{1}\right)+F_{2}\left(x_{2}\right)-1 \geq F_{1}\left(x_{1}\right)+F_{2}\left(x_{2}\right)-1,
$$

os limites de Fréchet para funções de distribuição bivariadas são

$$
\max \left\{F_{1}\left(x_{1}\right)+F_{2}\left(x_{2}\right)-1,0\right\} \leq F\left(x_{1}, x_{2}\right) \leq \min \left\{F_{1}\left(x_{1}\right), F_{2}\left(x_{2}\right)\right\},
$$

que em termos de cópula resulta em

$$
\max \left\{u_{1}+u_{2}-1,0\right\} \leq C_{\alpha}\left(u_{1}, u_{2}\right) \leq \min \left\{u_{1}, u_{2}\right\}, \quad u_{1}, u_{2} \in[0,1] .
$$

Finalizamos esta seção com duas outras propriedades associadas a cópulas. Elas são uniformemente contínuas e tem derivadas parciais de primeira ordem limitadas. 
Teorema 1.2.2

Dada uma cópula $C_{\alpha}$,

(a) $C_{\alpha}$ satisfaz a condição de Lipshitz

$$
\left|C_{\alpha}\left(u_{1}, u_{2}\right)-C_{\alpha}\left(v_{1}, v_{2}\right)\right| \leq\left|u_{1}-u_{2}\right|-\left|v_{1}-v_{2}\right|
$$

para $u_{1}, u_{2}, v_{1}, v_{2} \in[0,1]$.

(b) $C_{\alpha}$ é uniformemente contínua em $[0,1]^{2}$.

(c) As derivadas parciais

$$
\frac{\partial C_{\alpha}\left(u_{1}, u_{2}\right)}{\partial u_{1}} \in[0,1] \text { e } \frac{\partial C_{\alpha}\left(u_{1}, u_{2}\right)}{\partial u_{2}} \in[0,1]
$$

existem para cada par $\left(u_{1}, u_{2}\right) \in[0,1]^{2}$ e são limitadas.

Para a prova do teorema veja Nelsen (1999) ou Owzar e Sen (2003).

\subsection{Cópulas Arquimedianas}

Existe uma grande variedade de famílias de cópulas. A possibilidade de construí-las a partir de distribuições multivariadas e a especificação de marginais ad-hoc deixa claro que a lista de cópulas pode ser extensa. Exemplos de famílias de cópula são as classes de cópulas de Marshall-Olkin, elípticas e Arquimedianas, (veja Embrechts et al., 2003).

A cópula de Marshall-Olkin está relacionada com os tempos de vida exponenciais de dois componentes quando as falhas seguem très processos de Poisson independentes, em que os dois primeiros estão associados a cada 
componente, e o terceiro corresponde à possibilidade de falha simultânea dos componentes (distribuição com componente singular ${ }^{1}$ ).

Cópulas elípticas correspondem às cópulas de distribuições elípticas (veja por exemplo Fang, Kotz e Ng; 1987).

A familia de cópulas chamada Arquimedianas (Genest e MacKay, 1986, Nelsen, 1999, Capítulo 4), está relacionada aos modelos de sobrevivência bivariados e aos modelos multiplicativos de fragilidade, como será visto no próxima capítulo. A seguir definimos cópula Arquimediana.

\section{Definição 1.3.1}

Uma cópula $C_{\alpha}$ é dita ser Arquimediana se existir uma função convexa $\phi$ : $[0, \infty] \mapsto[0,1], \operatorname{com} \phi(0)=1, \phi^{\prime}<0, \phi^{\prime \prime}>0$, tal que a cópula $C_{\alpha}$ é expressa como

$$
C_{\alpha}\left(u_{1}, u_{2}\right)=\phi\left(\phi^{-1}\left(u_{1}\right)+\phi^{-1}\left(u_{2}\right)\right)
$$

para todo $\left(u_{1}, u_{2}\right) \in[0,1]^{2}$ e $\alpha \in \mathcal{A}$. A função de distribuição bivariada com marginais contínuas é dita ser uma cópula Arquimediana se sua cópula geradora é uma cópula Arquimediana. Neste caso, a função $\phi^{-1}$ é chamada de função geradora.

Além de revisar propriedades desta classe de funções; Genest e MacKay (1986) estabelecem condições em que estas distribuições possuem componentes singulares.

\footnotetext{
${ }^{1}$ L'ma variável aleatória $X \sim F$ é singular se, e somente se. $F$ é contínua e existe um conjunto $B$ de medida de Lebesgue zero tal que $\operatorname{Pr}\{X \in B\}=1$. veja por exemplo James (2004).
} 
A classe de cópulas Arquimedianas abrange um grande número de distribuições paramétricas bivariadas como, por exemplo, as famílias de Clayton, Estável Positiva (também conhecida como Gumbel) e Frank. Uma lista mais completa é dada em Joe (1997) e Nelsen (1999). Para estas três famílias, o gerador $\phi^{-1}$ é uma função derivável que tem um único parâmetro $\alpha \in \mathcal{A}$ que mede a força da dependência. Em cada caso, um valor particular ou um valor limite para $\alpha$ conduz ao gerador $\phi^{-1}(t)=-\ln (t)$ que implica independência.

\subsection{Tau de Kendall}

Cópulas provêem uma forma natural para medir a associação entre variáveis aleatórias, mas em geral, o parâmetro $\alpha$ que mede a dependência é difícil de interpretar. Uma forma alternativa para avaliar a associação é através da medida tau de Kendall, definida como

$\tau_{\alpha}\left(X_{1}, X_{2}\right)=\operatorname{Pr}\left\{\left(X_{1}^{*}-X_{1}\right)\left(X_{2}^{*}-X_{2}\right) \geq 0\right\}-\operatorname{Pr}\left\{\left(X_{1}^{*}-X_{1}\right)\left(X_{2}^{*}-X_{2}\right)<0\right\}$,

em que $\left(X_{1}, X_{2}\right)$ e $\left(X_{1}^{*}, X_{2}^{*}\right)$ são duas realizações independentes da distribuição conjunta cuja associação queremos avaliar.

Este coeficiente corresponde a probabilidade de concordância menos a probabilidade de disconcordância. Esta medida de associação satisfaz as propriedades de uma medida de concordancia, as quais podem ser consultadas, por exemplo. em Nelsen (1999). Seção 5.1.2. 
O seguinte teorema mostra a ligação entre o tau de Kendall e cópulas.

\section{Teorema 1.4.1}

Seja $\left(X_{1}, X_{2}\right)$ um vetor de variáveis aleatórias contínuas com cópula $C_{\alpha}$, então o coeficiente tau de Kendall para $\left(X_{1}, X_{2}\right)$ é dado por

$$
\begin{aligned}
\tau_{\alpha}\left(X_{1}, X_{2}\right) & =4 \mathrm{E}\left[F\left(X_{1}, X_{2}\right)\right]-1 \\
& =4 \iint_{[0,1]^{2}} C_{\alpha}\left(u_{1}, u_{2}\right) d C_{\alpha}\left(u_{1}, u_{2}\right)-1
\end{aligned}
$$

No seguinte teorema, dado em Genest e MacKay (1986), estabelece-se que para cópulas Arquimedianas o tau de Kendall pode ser expresso em termos da integral apenas de $\phi$ e de sua derivada.

\section{Teorema 1.4.2}

Sejam $X_{1}$ e $X_{2}$ variáveis aleatórias com cópula Arquimediana $C_{\alpha}$ gerada por $\phi$. Então o tau de Kendall de $X_{1}$ e $X_{2}{ }^{\prime}$ é dado por

$$
\tau_{\alpha}\left(X_{1}, X_{2}\right)=4 \int_{0}^{1} \frac{\phi^{-1}(t)}{\phi^{-1^{\prime}}(t)} d t+1
$$

Extensões multivariadas de cópulas arquimedianas são discutidas em Joe (1997), Nelsen (1999) e Embrechts et al. (2003). 


\subsection{Objetivos da Tese}

O presente trabalho objetiva modelar, sob a ótica Bayesiana, dados de sobrevivência bivariados através de cópulas.

É proposta uma metodologia de modelagem e estimação Bayesiana tanto da associação entre os tempos bivariados de sobrevivência, como para os parâmetros associados às covariáveis de interesse.

Através de uma aplicação do Teorema Central do Limite para funcionais são estabelecidas propriedades assintóticas da cópula como estimador da função de sobrevivência bivariada.

No Capítulo 2 desenvolvemos a estimação Bayesiana do modelo de sobrevivência com tempos dependentes. A metodologia é aplicada a dois exemplos populares na literatura de Análise de Sobrevivência.

Considerando a cópula como um funcional das funções de sobrevivência marginal, no Capítulo 3 são estudadas propriedades assintóticas do estimador Bayesiano da função de sobrevivência bivariada. Utilizando argumentos de convergência de estimadores obtidos com base em métodos de estimação via MCMC, de continuidade e o método delta para funcionais, consistência e convergência fraca são estabelecidas. 


\section{Capítulo 2}

\section{Modelagem e Estimação}

\subsection{Introdução}

Neste capítulo abordamos uma metodologia de modelagem baseada em cópulas para dados bivariados em Análise de Sobrevivência.

Estudamos a ligação entre os modelos de fragilidade multiplicativa e a classe de cópulas Arquimedianas. Posteriormente, procedimentos Bayesianos de estimação são propostos. Finalmente, duas aplicações são feitas exemplificando a metodologia descrita.

\subsection{Modelos de Fragilidade e Cópulas Arqui- medianas}

A seguir revisamos a relação entre os modelos de sobrevivência com fragilidade e a família de cópulas Arquimedianas dada em Oakes (1989). 
Os modelos de fragilidade são caracterizados pela introdução de um efeito aleatório (fragilidade) na função de taxa de falha com o objetivo de controlar a heterogeneidade não observável das unidades em estudo. Estes modelos foram apresentados inicialmente em Clayton (1978) e Vaupel, Manton e Stallard (1979). Clayton (1978) propôs uma extensão do modelo de Cox (1972) introduzindo, multiplicativamente, um efeito aleatório na função de taxa de falha para analisar a tendência familiar na incidência de doenças crônicas.

Considere $T$ uma variável aleatória contínua não negativa com função de sobrevivência $S$. Em um modelo de fragilidade multiplicativo tem-se que para $W$, variável aleatória não negativa representando um efeito aleatório, a função de taxa de falha e de sobrevivência, condicional a $W=w$, são dadas por

$$
\lambda(t \mid w)=\lambda_{0}(t) w, \quad \text { e } \quad S(t \mid w)=\exp \left(-\Lambda_{0}(t) w\right)=S_{0}(t)^{w}
$$

em que $\lambda_{0}, \Lambda_{0}$ e $S_{0}$ representam a função de taxa de falha, função de taxa de falha acumulada e função de sobrevivência basal, respectivamente.

A função de sobrevivência não condicional de $T$ é dada por

$$
\begin{aligned}
S(t) & =\mathrm{E}[S(t \mid W)] \\
& =\mathrm{E}\left[\exp \left(W \ln S_{0}(t)\right)\right] \\
& =p\left[-\ln S_{0}(t)\right]
\end{aligned}
$$

em que $p[u]=\mathrm{E}\left[e^{-u W}\right]=\int e^{-u w} f(w) d w$ é a transformada de Laplace de IV.

Considerando que $S^{\prime}(t)=p^{\prime}\left[-\ln S_{0}(t)\right] \lambda_{0}(t)$ note que a função de taxa de 
falha não condicional de $T$ é dada por

$$
\lambda(t)=-\frac{p^{\prime}\left[\Lambda_{0}(t)\right]}{p\left[\Lambda_{0}(t)\right]} \lambda_{0}(t) .
$$

Considere agora $\left(T_{1}, T_{2}\right)$ variáveis aleatórias contínuas não negativas com funções de sobrevivência dadas por $\left(S_{1}, S_{2}\right)$, representando tempos de falha bivariados, condicionalmente independentes dada a variável aleatória $W$, i.e., $S\left(t_{1}, t_{2} \mid w\right)=S\left(t_{1} \mid w\right) \cdot S\left(t_{2} \mid w\right)$. Então, a função de sobrevivência conjunta pode ser escrita como

$$
\begin{aligned}
S\left(t_{1}, t_{2}\right) & =\mathrm{E}\left[S\left(t_{1}, t_{2} \mid W\right)\right] \\
& =\mathrm{E}\left[\left(S_{1}\left(t_{1}\right) \cdot S_{2}\left(t_{2}\right)\right)^{W}\right] \\
& =p\left[-\ln \left(S_{1}\left(t_{1}\right) \cdot S_{2}\left(t_{2}\right)\right)\right] \\
& =p\left[q\left[S_{1}\left(t_{1}\right)\right]+q\left[S_{2}\left(t_{2}\right)\right]\right],
\end{aligned}
$$

em que $q\left[S_{j}\left(t_{j}\right)\right]=\Lambda_{j}\left(t_{j}\right)$ corresponde à função de taxa de falha acumulada, condicional a $W, j=1,2$. Quando $p[\cdot]$ é a transformada de Laplace de $W$ e $q[\cdot]$ é a sua respectiva função inversa, temos a distribuição bivariada de fragilidade, isto é, as distribuições bivariadas geradas a partir de modelos de fragilidade são uma subclasse de cópulas Arquimedianas (Oakes, 1989). Portanto,

$$
S\left(t_{1}, t_{2}\right)=\phi\left[\phi^{-1}\left(S_{1}\left(t_{1}\right)\right)+\phi^{-1}\left(S_{2}\left(t_{2}\right)\right)\right] .
$$

Considere agora a função de razões cruzadas (cross-ratio function) definida por

$$
\theta^{*}\left(t_{1}, t_{2}\right)=\frac{S\left(t_{1}, t_{2}\right) D_{1} D_{2} S\left(t_{1}, t_{2}\right)}{\left(D_{1} S\left(t_{1}, t_{2}\right)\right)\left(D_{2} S\left(t_{1}, t_{2}\right)\right)}=\frac{\lambda\left(t_{1} \mid t_{2}\right)}{\lambda\left(t_{1} \mid T_{2}>t_{2}\right)}
$$


em que $D_{j}$ denota $-\partial / \partial t_{j}$ e $\lambda(\cdot \mid \cdot)$ são funções de taxa de falha condicional.

Esta função foi introduzida por Clayton (1978) e diferentemente do coeficiente tau de Kendall, é uma medida de dependência local (dependente do tempo). Pode ser interpretado como a taxa de falha relativa $\theta^{*}$ para um indivíduo se o outro já experimentou o evento, em contraposição a estar livre do evento, em um determinado momento. Clayton considerou esta dependência constante em $\left(t_{1}, t_{2}\right)$, mas em certos problemas pode ser mais apropriado assumir que ela seja decrescente com o tempo quando este cresce (e.g. quando $T_{1}$ é o tempo de recidiva de alguma doença e $T_{2}$ o tempo até a morte, para um sujeito).

Algumas propriedades e comparações de $\theta^{*}\left(t_{1}, t_{2}\right)$ com outras medidas de dependência podem ser vistas em Anderson, Louis, Holm e Harvald (1992). Medidas de dependência local, que incluem a função de razões cruzadas como um caso particular, são estudadas em Fan, Prentice e Hsu (2000).

Para cópulas Arquimedianas, Oakes (1989) mostrou que a função de razões cruzadas depende em $\left(t_{1}, t_{2}\right)$ somente através de $S\left(t_{1}, t_{2}\right)$, tal que $\theta^{*}\left(t_{1}, t_{2}\right)=\theta\left(S\left(t_{1}, t_{2}\right)\right)$, em que a função $\theta$ define unicamente a $p[\cdot]$ dada em (2.2.1). O teorema seguinte dado em Oakes (1989) expressa o resultado.

\section{Teorema 2.2.1}

Suponha que $S\left(t_{1}, t_{2}\right)$ seja uma função de sobrevivência bivariada absolutamente contínua cuja função de razões cruzadas $\theta^{*}\left(t_{1} \cdot t_{2}\right)$ é expressável como $\theta\left(S\left(t_{1}, t_{2}\right)\right)$ para alguma função $\theta(v)$. Então, $S\left(t_{1}, t_{2}\right)$ satisfaz a representação 
(2.2.1), com $p[u]$ determinada implicitamente por

$$
q_{k}(v)=\int_{v}^{1} \exp \left\{\int_{z}^{1-k} \frac{\theta(y)}{y} d y\right\} d z
$$

em que

$$
\theta(v)=-v \frac{q^{\prime \prime}[v]}{q^{\prime}[v]}
$$

Note que em (2.2.3) a quantidade $k$ é um fator de escala, irrelevante para $p[\cdot]$.

Portanto, temos que qualquer modelo de fragilidade multiplicativo para dados de sobrevivência bivariados pode ser expresso por uma representação via cópula Arquimediana. Nas seções seguintes, este fato é ilustrado para três famílias de distribuições.

\subsubsection{Família de Clayton}

Clayton (1978) assume função de razões cruzadas constante e obtém o modelo de fragilidade gama. Considerando

$$
\theta(v)=\alpha+1, \alpha \in \mathbb{R}^{+},
$$

obtemos, a partir de (2.2.3) que $q[v]=\alpha^{-1}\left(v^{-\alpha}-1\right)$ e, portanto, $p[u]=$ $(1+\alpha u)^{-1 / \alpha}$, correspondendo à transformada de Laplace da distribuição gama. com ambos parâmetros iguais a $\alpha^{-1}$. De (2.2.1) obtemos que a função de sobrevivência conjunta é dada por

$$
S\left(t_{1} \cdot t_{2}\right)=\left(S_{1}\left(t_{1}\right)^{-\alpha}+S_{2}\left(t_{2}\right)^{-\alpha}-1\right)^{-\frac{1}{\alpha}} .
$$


Note que se $\alpha \rightarrow 0$, obtemos $S\left(t_{1}, t_{2}\right)=S_{1}\left(t_{1}\right) S_{2}\left(t_{2}\right)$.

O coeficiente tau de Kendall avaliado de (1.4.3) é dado por

$$
\tau_{\alpha}\left(T_{1}, T_{2}\right)=\frac{\alpha}{\alpha+2}
$$

\subsubsection{Fragilidade Estável Positiva}

Introduzida por Hougaard (1986a,b), esta distribuição de fragilidade tem a característica de manter invariante a propriedade do modelo de taxas de falha proporcionais. Considerando $\alpha \in(0,1)$, chamado de coeficiente de estabilidade, a transformada de Laplace é $p[u]=\exp \left(-u^{\alpha}\right)$, (e.g. veja Feller, 1971), que implica $q[v]=(-\ln v)^{1 / \alpha}$. A função de razões cruzadas obtida de (2.2.4), é dada por

$$
\theta(v)=1-\frac{1-\alpha}{\alpha \ln v}
$$

Note que esta função é decrescente em $t_{1}$ e $t_{2}$. A função de sobrevivência conjunta é

$$
S\left(t_{1}, t_{2}\right)=\exp \left\{-\left[\left(-\ln \left(S_{1}\left(t_{1}\right)\right)\right)^{1 / \alpha}+\left(-\ln \left(S_{2}\left(t_{2}\right)\right)\right)^{1 / \alpha}\right]^{\alpha}\right\},
$$

e se $\alpha \rightarrow 1$, obtemos $S\left(t_{1}, t_{2}\right)=S_{1}\left(t_{1}\right) S_{2}\left(t_{2}\right)$. O tau de Kendall é dado por

$$
\tau_{\alpha}\left(T_{1}, T_{2}\right)=1-\alpha
$$

Aplicações de modelos de fragilidade em que o efeito aleatório tem distribuição Estável Positiva dentro de uma perspectiva Bayesiana podem ser vistas em Ibrahim, Chen e Sinha (2001). 


\subsubsection{Família de Frank}

Esta classe de distribuições foi estudada por Frank (1979) no contexto de espaços métricos probabilísticos. Propriedades estatísticas desta família são revisadas em Nelsen (1986) e Genest (1987). A função de distribuição da família de Frank, para $\alpha \neq 1$, é dada por,

$$
\operatorname{Pr}\left\{T_{1} \leq t_{1}, T_{2} \leq t_{2}\right\}=\log _{\alpha}\left(1+\frac{\left(\alpha^{t_{1}}-1\right)\left(\alpha^{t_{2}}-1\right)}{\alpha-1}\right),
$$

em que $\log _{\alpha}$ denota o logaritmo na base $\alpha$. Quando o parâmetro de dependência $\alpha \in(0,1)$, esta distribuição tem transformada de Laplace $p[u]=$ $\log _{\alpha}\left(1-(1-\alpha) e^{-u}\right)$. A função inversa de $p[\cdot]$ é dada por $q[v]=-\ln \left(\frac{1-\alpha^{v}}{1-\alpha}\right)$, e a sua respectiva função de razões cruzadas é

$$
\theta(v)=-v \frac{\ln \alpha}{1-\alpha^{v}}
$$

Note que $\theta(v)=\theta\left(S\left(t_{1}, t_{2}\right)\right)$ é decrescente em $t_{1}$ e $t_{2}$.

A função de sobrevivência conjunta é

$$
S\left(t_{1}, t_{2}\right)=\log _{\alpha}\left(1+\frac{\left(\alpha^{S_{1}\left(t_{1}\right)}-1\right)\left(\alpha^{S_{2}\left(t_{2}\right)}-1\right)}{\alpha-1}\right),
$$

tal que se $\alpha \rightarrow 1, S\left(t_{1}, t_{2}\right)=S_{1}\left(t_{1}\right) S_{2}\left(t_{2}\right)$. O tau de Kendall é dado por

$$
\tau_{\alpha}\left(T_{1}, T_{2}\right)=1+\frac{4}{\ln \alpha}\left(\frac{1}{\ln \alpha} \int_{0}^{-\ln \alpha} \frac{t}{e^{t}-1} d t+1\right) .
$$

Uma característica importante desta família é que se consideramos a reparametrização $\alpha=e^{-\gamma}$, com $\gamma \neq 0$, temos que, diferentemente das outras distribuições já vistas anteriormente que só consicleram associação positiva, este modelo captura todo o campo de variação da associação, tanto positiva como negativa. 
A transformada de Laplace neste caso é $p[u]=-(1 / \gamma) \ln \left(1+\left(e^{-\gamma}-1\right) e^{-u}\right)$, e $q[v]=-\ln \left(\frac{e^{-\gamma v}-1}{e^{-\gamma}-1}\right)$. A função de razões cruzadas é dada por

$$
\theta(v)=-v \frac{\gamma}{e^{-\gamma v}-1} .
$$

Note que $\theta(v)=\theta\left(S\left(t_{1}, t_{2}\right)\right)$ é decrescente em $t_{1}$ e $t_{2}$ se $\gamma>0$, e crescente para $\gamma<0$.

A função de sobrevivência conjunta é

$$
S\left(t_{1}, t_{2}\right)=-\frac{1}{\gamma} \ln \left(1+\frac{\left(e^{-\gamma S_{1}\left(t_{1}\right)}-1\right)\left(e^{-\gamma S_{2}\left(t_{2}\right)}-1\right)}{e^{-\gamma}-1}\right),
$$

e o tau de Kendall é dado por

$$
\tau_{\gamma}\left(T_{1}, T_{2}\right)=1-\frac{4}{\gamma}\left(1-\frac{1}{\gamma} \int_{0}^{\gamma} \frac{t}{e^{t}-1} d t\right),
$$

tal que $\tau_{\gamma}\left(T_{1}, T_{2}\right)=-\tau_{-\gamma}\left(T_{1}, T_{2}\right)$, isto é, para valores negativos de $\gamma$, a associação entre os tempos $\left(T_{1}, T_{2}\right)$ é inversa.

\subsection{Estimação Bayesiana}

Na seção anterior apresentamos a estrutura básica de modelos de cópula e sua relação com os modelos de fragilidade. Como a escolha das distribuições marginais não depende da escolha de uma particular cópula, faz sentido considerar a estimação das marginais e o parâmetro de dependência separadamente. Assim, as inferências sobre este parâmetro estariam livres do efeito das distribuições marginais consideradas.

Em Genest. Ghoudi e Rivest (1995) é considerada uma estimação semi-paramétrica clássica para o paràmetro de dependência, onde uma 
pseudo-verossimilhança é maximizada e as distribuições marginais são substituídas pelas respectivas funções de distribuição empíricas.

A ocorrência de censura é considerada por Shih e Louis (1995), e a estimação é desenvolvida em duas etapas: na primeira assume-se independência entre os tempos de falha e as funções de sobrevivência marginais são estimadas usando o estimador de Kaplan-Meier. Eles também consideram uma família paramétrica para as marginais. Na segunda etapa o parâmetro de associação é estimado assumindo as marginais estimadas como fixas.

A seguir apresentamos o procedimento de estimação.

Sejam $\left(T_{1}, T_{2}\right)$ tempos de falha bivariados com funções de sobrevivência $\left(S_{1}, S_{2}\right)$ e densidades $\left(f_{1}, f_{2}\right)$. Considere também $\left(C_{1}, C_{2}\right)$ tempos de censura bivariados. Para $i=1, \ldots, n$ suponha que $\left(T_{i 1}, T_{i 2}\right)$ e $\left(C_{i 1}, C_{i 2}\right)$ são independentes. Para cada $i$ as quantidades observadas são representadas pelas variáveis aleatórias $Z_{i j}=\min \left\{T_{i j}, C_{i j}\right\}$ e $\delta_{i j}=I\left[Z_{i j}=T_{i j}\right], j=1,2$.

Para realizar o procedimento Bayesiano de estimação paramétrica em duas etapas, suponha que uma família paramétrica de distribuições seja conhecida para os tempos de falha marginais e sejam $\theta_{1}$ e $\theta_{2}$ vetores de parâmetros associados a cada uma das distribuições marginais. Nós propomos estimar as marginais separadamente em uma primeira etapa, assumindo independência entre os tempos de falha e considerando distribuições a priori para os parâmetros $\boldsymbol{\theta}_{1}$ e $\boldsymbol{\theta}_{2}$ dadas por $\pi_{j}^{\theta}(\cdot), j=1.2$ e função de verossimilhança 


$$
L\left(\theta_{j} \mid z_{j}, \delta_{j}\right)=\prod_{i=1}^{n} f_{j \theta_{j}}\left(z_{i j}\right)^{\delta_{i j}} S_{j \theta_{j}}\left(z_{i j}\right)^{1-\delta_{i j},} \quad j=1,2,
$$

obtendo assim a distribuição a posteriori

$$
\pi\left(\theta_{j} \mid z_{j}, \delta_{j}\right) \propto L\left(\theta_{j} \mid z_{j}, \delta_{j}\right) \pi_{j}^{\theta}\left(\boldsymbol{\theta}_{j}\right)
$$

Se $\widetilde{\theta}_{j}$ é o estimador de $\boldsymbol{\theta}_{j}$ obtido com base na distribuição a posteriori (2.3.2) em relação a alguma função de perda, o estimador de $\alpha$ (obtido na etapa 2) é obtido a partir da "distribuição a posteriori"

$$
\pi\left(\alpha \mid z_{1}, z_{2}, \boldsymbol{\delta}_{1}, \boldsymbol{\delta}_{2}, \widetilde{\boldsymbol{\theta}}_{1}, \widetilde{\boldsymbol{\theta}}_{2}\right) \propto L\left(\alpha \mid z_{1}, \boldsymbol{z}_{2}, \boldsymbol{\delta}_{1}, \boldsymbol{\delta}_{2}, \widetilde{\boldsymbol{\theta}}_{1}, \widetilde{\boldsymbol{\theta}}_{2}\right) \pi^{\alpha}(\alpha)
$$

com base na pseudo-verossimilhança

$$
\begin{gathered}
L\left(\alpha \mid \boldsymbol{z}_{1}, \boldsymbol{z}_{2}, \boldsymbol{\delta}_{1}, \boldsymbol{\delta}_{2}, \widetilde{\boldsymbol{\theta}}_{1}, \widetilde{\boldsymbol{\theta}}_{2}\right)=\prod_{i=1}^{n} f\left(z_{i 1}, z_{i 2} ; \alpha\right)^{\delta_{i 1} \delta_{i 2}} \frac{\partial S\left(z_{i 1}, z_{i 2} ; \alpha\right)^{\delta_{i 1}\left(1-\delta_{i 2}\right)}}{\partial z_{i 1}} \\
. \frac{\partial S\left(z_{i 1}, z_{i 2} ; \alpha\right)^{\delta_{i 2}\left(1-\delta_{i 1}\right)}}{\partial z_{i 2}} S\left(z_{i 1}, z_{i 2} ; \alpha\right)^{\left(1-\delta_{i 1}\right)\left(1-\delta_{i 2}\right)},
\end{gathered}
$$

em que $S\left(z_{i 1}, z_{i 2} ; \alpha\right)=C_{\alpha}\left(S_{1 \tilde{\theta}_{1}}\left(z_{i 1}\right), S_{2 \tilde{\theta}_{2}}\left(z_{i 2}\right)\right)$ é a função de sobrevivência bivariada, $f\left(z_{i 1}, z_{i 2} ; \alpha\right)=\frac{\partial^{2} S\left(z_{i 1}, z_{i 2} ; \alpha\right)}{\partial z_{i 1} \partial z_{i 2}}$ é a correspondente função de densidade bivariada, e supondo distribuição a priori para $\alpha$ dada por $\pi^{\alpha}(\cdot)$.

Para realizar uma estimação Bayesiana semiparamétrica em duas etapas, estimamos na primeira etapa as funções de sobrevivência marginal supondo independência. Assim, considere $\widetilde{S}_{j}, j=1,2$ ser algum estimador Bavesiano não paramétrico ${ }^{1}$ de $S_{j}$. Então, dados vetores $\left(u_{1}, u_{2}\right)$ com elementos $\left(u_{i 1}, u_{i 2}\right)=\left(\widetilde{S}_{1}\left(Z_{i 1}\right), \widetilde{S}_{2}\left(Z_{i 2}\right)\right), i=1 \ldots, n$, o estimador de a na etapa

\footnotetext{
${ }^{1}$ Algums exemplos de tais estimadores são dados em Susarla e Van Ryzin (1976). Hjört (1990) e Salinas-Torres, Pereira e Tiwari (2002).
} 
2 é calculado a partir da seguinte "distribuição a posteriori"

$$
\pi\left(\alpha \mid \delta_{1}, \delta_{2}, u_{1}, u_{2}\right) \propto L\left(\alpha \mid \delta_{1}, \delta_{2}, u_{1}, u_{2}\right) \pi(\alpha)
$$

obtida com base na função de pseudo-verossimilhança

$$
\begin{aligned}
L\left(\alpha \mid \delta_{1}, \delta_{2}, u_{1}, u_{2}\right) & =\prod_{i=1}^{n} c_{\alpha}\left(u_{i 1}, u_{i 2}\right)^{\delta_{i 1} \delta_{i 2}} \frac{\partial C_{\alpha}\left(u_{i 1}, u_{i 2}\right)^{\delta_{i 1}\left(1-\delta_{i 2}\right)}}{\partial u_{i 1}} \\
& \cdot \frac{\partial C_{\alpha}\left(u_{i 1}, u_{i 2}\right)^{\delta_{i 2}\left(1-\delta_{i 1}\right)}}{\partial u_{i 2}} C_{\alpha}\left(u_{i 1}, u_{i 2}\right)^{\left(1-\delta_{i 1}\right)\left(1-\delta_{i 2}\right)} .
\end{aligned}
$$

No caso de uma estimação conjunta para as marginais e o parâmetro de dependência, considerando o enfoque Bayesiano, suponha que $\left(T_{1}, T_{2}\right)$ tem funções marginais de sobrevivência e densidade $\left(S_{1 \theta_{1}}, S_{2 \theta_{2}}\right)$ e $\left(f_{1 \theta_{1}}, f_{2 \theta_{2}}\right)$, respectivamente. A distribuição a posteriori conjunta pode ser escrita como

$$
\pi\left(\alpha, \boldsymbol{\theta}_{1}, \boldsymbol{\theta}_{2} \mid \boldsymbol{z}_{1}, \boldsymbol{z}_{2}, \boldsymbol{\delta}_{1}, \boldsymbol{\delta}_{2}\right) \propto L\left(\alpha, \boldsymbol{\theta}_{1}, \boldsymbol{\theta}_{2} \mid \boldsymbol{z}_{1}, \boldsymbol{z}_{2}, \boldsymbol{\delta}_{1}, \boldsymbol{\delta}_{2}\right) \pi\left(\alpha, \boldsymbol{\theta}_{1}, \boldsymbol{\theta}_{2}\right),
$$

com base na função de verossimilhança

$$
\begin{aligned}
L\left(\alpha, \theta_{1}, \boldsymbol{\theta}_{2} \mid z_{1}, z_{2}, \boldsymbol{\delta}_{1}, \boldsymbol{\delta}_{2}\right) & =\prod_{i=1}^{n}\left(c_{\alpha}\left(S_{1 \theta_{1}}\left(z_{i 1}\right), S_{2 \theta_{2}}\left(z_{i 2}\right)\right) f_{1 \theta_{1}}\left(z_{i 1}\right) f_{2 \theta_{2}}\left(z_{i 2}\right)\right)^{\delta_{i 1} \delta_{i 2}} \\
& \cdot\left(\frac{\partial C_{\alpha}\left(S_{1 \theta_{1}}\left(z_{i 1}\right), S_{2 \theta_{2}}\left(z_{i 2}\right)\right)}{\partial S_{1 \theta_{1}}\left(z_{i 1}\right)} \cdot\left(-f_{1 \theta_{1}}\left(z_{i 1}\right)\right)\right)^{\delta_{i 1}\left(1-\delta_{i 2}\right)} \\
& \cdot\left(\frac{\partial C_{\alpha}\left(S_{1 \theta_{1}}\left(z_{i 1}\right), S_{2 \theta_{2}}\left(z_{i 2}\right)\right)}{\partial S_{2 \theta_{2}}\left(z_{i 2}\right)} \cdot\left(-f_{2 \theta_{2}}\left(z_{i 2}\right)\right)\right)^{\left(1-\delta_{i 1}\right) \delta_{i 2}} \\
& \cdot C_{\alpha}\left(S_{1 \theta_{1}}\left(z_{i 1}\right), S_{2 \theta_{2}}\left(z_{i 2}\right)\right)^{\left(1-\delta_{i 1}\right)\left(1-\delta_{i 2}\right)}
\end{aligned}
$$

e distribuição a priori para $\left(\alpha, \theta_{1}, \theta_{2}\right)$ dada por $\pi(\because \cdot \cdot)$.

Note que na especificação paramétrica das marginais é possivel introduzir corariáveis em cada um dos componentes, i.e., $\theta_{j}=v\left(\mathrm{X}_{j}\right)$. Por simplicidade e 
para evitar restrições no espaço paramétrico considere $\psi\left(\mathbf{X}_{j}\right)=\exp \left(\mathbf{X}_{j}^{T} \boldsymbol{\beta}_{j}\right)$,

$\operatorname{com} \theta_{j} \in \theta_{j}$, e $\boldsymbol{\beta}_{j}$ correspondendo ao vetor de coeficientes desconhecidos, associados as covariáveis $\mathrm{X}_{j}, j=1,2$.

\subsection{Aplicações}

Nesta seção discutimos a aplicação dos métodos de estimação propostos na seção anterior. Em particular será ilustrado o procedimento paramétrico em duas etapas, e o procedimento de estimação conjunta.

Dois conjuntos de dados são analisados. O primeiro conjunto de dados corresponde a sujeitos com retinopatia diabética e o segundo a pacientes com leucemia.

Os três modelos estudados na Seção 2.2 são ajustados ao primeiro conjunto de dados. Através da aplicação de quatro métodos, um não paramétrico, dois baseados na distribuição preditiva, e o criterio $B I C$, fazemos uma comparação entre modelos.

Para o segundo conjunto de dados ajustamos o modelo de Frank reparametrizado dado em (2.2.8), ilustrando o modelagem de associação negativa entre tempos de falha.

\subsubsection{Pacientes com Retinopatia Diabética}

Os dados consistem dos tempos de acompanhamento de 197 pacientes diabéticos com retinopatia com menos de 60 anos de idade nos Estados Unidos. O evento de interesse é a perda da acuidade visual. O objetivo principal do estudo é avaliar a eficácia do tratamento de fotocoagulação 
retardando o começo da perda da acuidade visual. O tratamento foi atribuído aleatoriamente a um olho de cada paciente. $\mathrm{O}$ outro olho foi considerado como controle. Então, o primeiro componente no vetor de tempos é o tempo até perda da acuidade visual no olho tratado $\left(T_{1}\right)$ e o segundo componente é o tempo até perda da acuidade visual no olho sem tratamento $\left(T_{2}\right)$. Os pacientes podem ser censurados, o que aconteceu para $73 \%$ dos olhos tratados e $49 \%$ dos olhos sem tratamento. Como covariável considerou-se a idade no diagnóstico da diabete, categorizada em 2 grupos, com ponto de corte de 20 anos ( $58 \%$ dos sujeitos foram considerados com menos de 20 anos de idade). Para essa covariável, 1 representa a categoria adulto e 0 a categoria jovem.

Estes dados foram analisados considerando-se uma abordagem freqüentista em Huster, Brookmeyer e Self (1989) através de um modelo que considera a cópula de Clayton com função de razões cruzadas $\theta(v)=\alpha, \alpha>1$, e marginais Weibull. Classicamente são analisados também em Therneau e Grambsch (2000), usando uma abordagem de modelos de efeitos aleatórios. A partir de uma perspectiva Bayesiana, Sahu e Dey (2000) consideram uma família de distribuições bivariadas exponencial e Weibull.

Na Figura 2.1 apresentam-se as curvas de sobrevivência de Kaplan-Meier para cada olho, separados por faixa etária. Observa-se que, aparentemente, o tratamento é mais efetivo para pacientes diagnosticados com diabetes já adultos.

Note também que para os individuos que receberam o tratamento, as curras de Kaplan-Meier sugerem a presença de uma proporção de sujeitos curados, pois os tempos maiores são censurados. 
Figura 2.1: Função de sobrevivência Kaplan-Meier dados de retinopatia diabética, Tempo 1: grupo com tratamento [esquerda], Tempo 2: grupo control [direita].
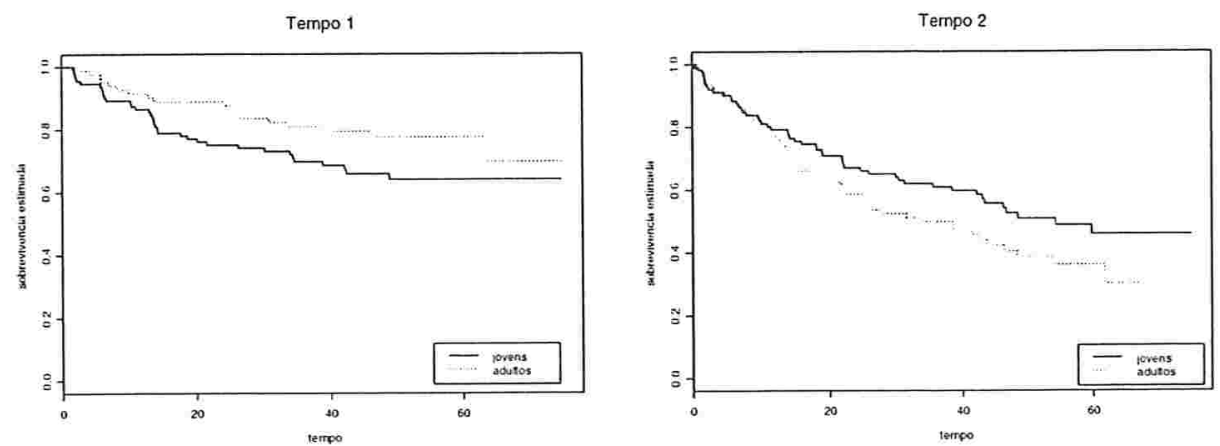

Para realizar a estimação paramétrica em duas etapas considere $T_{j} \sim$ Weibull $\left(r_{j}, \lambda_{j}\right)$ com função de densidade dada por

$$
f(t \mid r, \lambda)=r \lambda t^{r-1} e^{-\lambda t^{r}}, \quad r>0, \lambda>0
$$

A introdução de covariáveis se dá através de $\lambda=\exp \left(\beta_{0}+\beta_{1} X\right)$, isto é, supondo que o efeito das covariáveis atua proporcionalmente na função de taxa de falha.

Como distribuições a priori para os parâmetros da distribuição Weibull considere $r \sim \exp (0,001), \beta_{0} \sim \operatorname{Normal}\left(0,100^{2}\right)$ e $\beta_{1} \sim \operatorname{Normal}\left(0,100^{2}\right)$, isto é, distribuições a priori difusas ou vagas.

Seguindo Ibrahim et al. (2001), a distribuição a posteriori (2.3.2), com as distribuições a priori apontadas, é log-côncava e o algoritmo de rejeição adaptativo (Gilks e Wild, 1992) pode ser usado para amostrar da distribuição a posteriori. Este procedimento está implementado no programa WinBugs. versão 1.4.1 (Spiegelhalter, Thomas e Best, 2003). 
Depois de um burn-in de 1.000 valores, os resultados da estimação para cada tempo são obtidos e estão apresentados na Tabela 2.1, considerando $n=2.000$ valores amostrados. Na tabela, HDP corresponde ao intervalo de credibilidade com densidade (probabilidade) a posteriori máxima, o qual está implementado no programa BOA, versão 1.1.5 (Smith, 2005). Como critério de convergência, para todas as simulações apresentadas neste capítulo, usou-se o critério de Geweke (1992), também implementado no programa BOA.

Tabela 2.1: Média a posteriori, [desvio padrão] e (HDP 95\%), modelo Weibull.

\begin{tabular}{cccc}
\hline Tempo & $r$ & $\beta_{0}$ & $\beta_{1}$ \\
\hline 1 & $0,799[0,099]$ & $-4,003[0,406]$ & $-0,525[0,297]$ \\
& $(0,623 ; 1,005)$ & $(-4,801 ;-3,228)$ & $(-1,079 ; 0,090)$ \\
\hline 2 & $0,835[0,073]$ & $-3,701[0,306]$ & $0,353[0,201]$ \\
& $(0,692 ; 0,974)$ & $(-4,310 ;-3,096)$ & $(-0,027 ; 0,765)$ \\
\hline
\end{tabular}

Da Tabela 2.1 é possível dizer que o modelo Weibull é adequado para cada tempo, com os parâmetros estimados significativamente distintos de 1 e 0 , respectivamente. Note que o efeito da faixa etária no tempo de perda da acuidade visual é distinto conforme o tratamento. Se consideramos somente o olho tratado, o valor estimado para $\beta_{1}$ é negativo, sugerindo que os pacientes diabéticos com maior idade podem ser mais beneficiados com o tratamento. 
Seguindo com a etapa 2 da estimação considere a cópula Estável Positiva dada em (2.2.6) como estrutura de dependência para ser usada na pseudoverossimilhança em (2.3.4) e assim obter a "distribuição a posteriori" (2.3.3), considerando a priori $\alpha \sim \operatorname{Beta}(a, b)$. As funçōes de sobrevivência marginais a serem usadas em (2.3.4) são Weibull com estimativas $\widetilde{r}_{1}$ e $\widetilde{\lambda}_{1}=\exp \left(\widetilde{\beta}_{01}+\right.$ $\left.\widetilde{\beta}_{11} X\right)$ para $T_{1}$, e $\widetilde{r}_{2}$ e $\widetilde{\lambda}_{2}=\exp \left(\widetilde{\beta}_{02}+\widetilde{\beta}_{12} X\right)$ para $T_{2}$, em que $\left(\widetilde{\theta}_{1}, \widetilde{\theta}_{2}\right)=$ $\left(\widetilde{r}_{1}, \widetilde{\beta}_{01}, \widetilde{\beta}_{11} ; \widetilde{r}_{2}, \widetilde{\beta}_{02}, \widetilde{\beta}_{12}\right)$ são dadas pelas correspondentes médias a posteriori, isto é: $(0,799 ;-4,003 ;-0,525 ; 0,835 ;-3,701 ; 0,353)$.

Dadas as especificações anteriores a pseudo-verossimilhança em (2.3.4) pode ser escrita como

$$
\begin{aligned}
L\left(\alpha \mid z_{1}, z_{2}, \delta_{1}, \delta_{2}, \widetilde{\theta}_{1}, \widetilde{\theta}_{2}\right) \propto & \prod_{i=1}^{n} \alpha^{-\delta_{i 1} \delta_{i 2}} \widetilde{\lambda}_{1}^{\delta_{i 1} / \alpha} \widetilde{\lambda}_{2}^{\delta_{i 2} / \alpha}\left(z_{i 1}^{\widetilde{r}_{1} \delta_{i 1}} z_{i 2}^{\widetilde{2}_{2} \delta_{i 2}}\right)^{1 / \alpha-1} \\
& \cdot s_{\alpha i}^{\alpha\left(\delta_{i 1}+\delta_{i 2}-\delta_{i 1} \delta_{i 2}\right)-\delta_{i 1}-\delta_{i 2}} e^{-s_{\alpha i}^{\alpha}}\left(1-\alpha+\alpha s_{\alpha i}^{\alpha}\right)^{\delta_{i 1} \delta_{i 2}},
\end{aligned}
$$

$\operatorname{com} s_{\alpha i}=\left(\widetilde{\lambda}_{1} z_{i 1}^{\widetilde{r}_{1}}\right)^{1 / \alpha}+\left(\widetilde{\lambda}_{2} z_{i 2}^{\widetilde{r}_{2}}\right)^{1 / \alpha}$.

Logo, a "distribuição a posteriori" para o parâmetro de dependência é dada por

$$
\begin{aligned}
\pi\left(\alpha \mid z_{1}, z_{2}, \delta_{1}, \delta_{2}, \widetilde{\theta}_{1}, \widetilde{\theta}_{2}\right) \propto & \alpha^{a-\sum_{i} \delta_{i 1} \delta_{i 2}-1}(1-\alpha)^{b-1} \prod_{i=1}^{n} \widetilde{\lambda}_{1}^{\delta_{i 1} / \alpha} \widetilde{\lambda}_{2}^{\delta_{i 2} / \alpha} \\
& \cdot\left(z_{i 1}^{\tilde{r}_{1} \delta_{i 1}} z_{i 2}^{\tilde{r}_{2} \delta_{i 2}}\right)^{1 / \alpha-1} s_{\alpha i}^{\alpha\left(\delta_{i 1}+\delta_{i 2}-\delta_{i 1} \delta_{i 2}\right)-\delta_{i 1}-\delta_{i 2}} \\
& \cdot e^{-s_{\alpha i}^{\alpha}\left(1-\alpha+\alpha s_{\alpha i}^{\alpha}\right)^{\delta_{i 1} \delta_{i 2}}} .
\end{aligned}
$$

Esta expressão foi implementada no WinBugs 1.4.1, com $\alpha \sim \operatorname{Beta}(1.1)$ como distribuição a priori. usando-se o método de slice sampling para amostrar valores da distribuição a posteriori (reja Neal. 2003). Obtém-se. 
depois de um burn-in de 1.000 valores e uma amostra de 2.000 dados, que a média a posteriori do parâmetro de dependência é de 0,796 , com um desvio padrão de 0,051 e intervalo de credibilidade HDP $95 \%$ de $(0,704 ; 0,902)$. Assim, a estimativa para o tau de Kendall é 0,204.

Na Tabela 2.2 apresentam-se resultados da estimação em duas etapas para os modelos de cópula Estável Positiva, além dos resultados obtidos implementando as cópulas de Frank (2.2.7) e de Clayton (2.2.5). Também foram consideradas amostras de 2.000 valores e um burn-in de 1.000 dados. Para o modelo baseado na cópula de Frank considerou-se como distribuição a priori, $\alpha \sim \operatorname{Beta}(1,1)$ e para o modelo de Clayton considerou-se $\alpha \sim \operatorname{Gama}(1 ; 0,001)$.

Tabela 2.2: Média a posteriori, [desvio padrão] e (HDP 95\%), estimação em 2 etapas.

\begin{tabular}{ccc}
\hline cópula & $\alpha$ & $\tau_{\alpha}$ \\
\hline Estável & $0,796[0,051]$ & $0,204[0,051]$ \\
Positiva & $(0,704 ; 0,902)$ & $(0,098 ; 0,296)$ \\
\hline Frank & $0,153[0,101]$ & $0,218[0,060]$ \\
& $(0,027 ; 0,358)$ & $(0,106 ; 0,334)$ \\
\hline Clayton & $1,061[0,336]$ & $0,339[0,073]$ \\
& $(0,422 ; 1,718)$ & $(0,187 ; 0,469)$ \\
\hline
\end{tabular}

Para realizar o procedimento de estimação conjunta, consideramos $T_{j} \sim$ Weibull $\left(r_{j}, \lambda_{j}\right), \operatorname{com} \lambda_{j}=\exp \left(\beta_{0 j}+\beta_{1 j} X\right), j=1.2$. Para a cópula Estável Positiva, a função de verossimilhança em (2.3.7) pode ser escrita como 


$$
\begin{gathered}
L\left(\alpha, r_{1}, r_{2}, \boldsymbol{\beta}_{1}, \boldsymbol{\beta}_{2} \mid z_{1}, z_{2}, \delta_{1}, \delta_{2}, X\right) \propto \prod_{i=1}^{n} \alpha^{-\delta_{i 1} \delta_{i 2}} e^{\left(\delta_{i 1} / \alpha\right)\left(\beta_{01}+\beta_{11} X\right)} e^{\left(\delta_{i 2} / \alpha\right)\left(\beta_{02}+\beta_{12} X\right)} \\
\cdot z_{i 1}^{\delta_{i 1}\left(r_{1} / \alpha-1\right)} z_{i 2}^{\delta_{i 2}\left(r_{2} / \alpha-1\right)} r_{1}^{\delta_{i 1}} r_{2}^{\delta_{i 2}} s_{\alpha i}^{\alpha\left(\delta_{i 1}+\delta_{i 2}-\delta_{i 1} \delta_{i 2}\right)-\delta_{i 1}-\delta_{i 2}} e^{-s_{\alpha i}^{\alpha}}\left(1-\alpha+\alpha s_{\alpha i}^{\alpha}\right)^{\delta_{i 1} \delta_{i 2}}
\end{gathered}
$$

em que $s_{\alpha i}=\left(z_{i 1}^{r_{1}} e^{\left(\beta_{01}+\beta_{11} X\right)}\right)^{1 / \alpha}+\left(z_{i 2}^{r_{2}} e^{\left(\beta_{02}+\beta_{12} X\right)}\right)^{1 / \alpha}$, e $\beta_{j}=\left(\beta_{0 j}, \beta_{1 j}\right)$.

Considerando como prioris $\alpha \sim \operatorname{Beta}(a, b), r_{j} \sim \exp \left(r_{0 j}\right), \beta_{0 j} \sim$ $\operatorname{Normal}\left(c_{0 j}, d_{0 j}^{2}\right)$ e $\beta_{1 j} \sim \operatorname{Normal}\left(c_{1 j}, d_{1 j}^{2}\right)$, segue que a distribuição a posteriori conjunta para os parâmetros $\left(\alpha, r_{1}, r_{2}, \boldsymbol{\beta}_{1}, \boldsymbol{\beta}_{2}\right)$ é dada por

$$
\begin{gathered}
\pi\left(\alpha, r_{1}, r_{2}, \boldsymbol{\beta}_{1}, \boldsymbol{\beta}_{2} \mid \mathrm{z}_{1}, \mathbf{z}_{2}, \delta_{1}, \delta_{2}, X\right) \propto \alpha^{a-\sum_{i} \delta_{i 1} \delta_{i 2}-1}(1-\alpha)^{b-1} \\
\cdot e^{-r_{01} r_{1}-r_{02} r_{2}} e^{\sum_{i}\left(\beta_{01}+\beta_{11} X_{i}\right) \delta_{i 1} / \alpha-\left(\beta_{01}^{2}-2 \beta_{01} c_{01}\right) / 2 d_{01}^{2}-\left(\beta_{11}^{2}-2 \beta_{11} c_{01}\right) / 2 d_{11}^{2}} \\
\cdot e^{\sum_{i}\left(\beta_{02}+\beta_{12} X_{i}\right) \delta_{i 2} / \alpha-\left(\beta_{02}^{2}-2 \beta_{02} c_{02}\right) / 2 d_{02}^{2}-\left(\beta_{12}^{2}-2 \beta_{12} c_{02}\right) / 2 d_{12}^{2}} \prod_{i=1}^{n} z_{i 1}^{\delta_{i 1}\left(r_{1} / \alpha-1\right)} \\
\cdot z_{i 2}^{\delta_{i 2}\left(r_{2} / \alpha-1\right)} r_{1}^{\delta_{i 1}} r_{2}^{\delta_{i 2}} s_{\alpha i}^{\alpha\left(\delta_{i 1}+\delta_{i 2}-\delta_{i 1} \delta_{i 2}\right)-\delta_{i 1}-\delta_{i 2}} e^{-s_{\alpha i}^{\alpha}}\left(1-\alpha+\alpha s_{\alpha i}^{\alpha}\right)^{\delta_{i 1} \delta_{i 2}}
\end{gathered}
$$

A implementação da expressão (2.4.2) foi feita em WinBugs 1.4.1, com $\alpha \sim \operatorname{Beta}(1,1), r_{j} \sim \exp (0,001)$ e $\beta_{k j} \sim \operatorname{Normal}\left(0,100^{2}\right), k=0,1$, $j=1,2$ como prioris e usando o método de slice sampling e o algoritmo de Metropolis-Hastings (Metropolis et al., 1953; Hastings, 1970). Mostra-se nas Tabelas 2.3 e 2.4 as estimativas dos parâmetros, depois de um burn-in de 4.000 valores e uma amostra de 2.000 dados. Também são apresentadas as estimativas obtidas usando as cópulas de Frank e Clayton, considerando as mesmas especificações a priori da estimação em duas etapas.

Das Tabelas 2.3 e 2.4 notamos que há uma associação positiva significativa entre os tempos de perda da acuidade visual. Descle que a rariabilidade 
Tabela 2.3: Média a posteriori, [desvio padrão] e (HDP 95\%), estimação conjunta.

\begin{tabular}{ccccc}
\hline cópula & $3_{01}$ & $\beta_{11}$ & $\beta_{02}$ & $\beta_{12}$ \\
\hline Estável & $-3,957[0,400]$ & $-0,441[0,278]$ & $-3,641[0,296]$ & $0,355[0,197]$ \\
Positiva & $(-4,657 ;-3,103)$ & $(-0,987 ; 0,112)$ & $(-4,227 ;-3,101)$ & $(-0,043 ; 0,728)$ \\
\hline \multirow{2}{*}{ Frank } & $-4,075[0,415]$ & $-0,474[0,293]$ & $-3,709[0,303]$ & $0,372[0,197]$ \\
& $(-4,934 ;-3,320)$ & $(-1,060 ; 0,044)$ & $(-4,333 ;-3,143)$ & $(-0,015 ; 0,765)$ \\
\hline \multirow{2}{*}{ Clayton } & $-4,051[0,407]$ & $-0,477[0,290]$ & $-3,710[0,311]$ & $0,383[0,200]$ \\
& $(-4,942 ;-3,335)$ & $(-1,071 ; 0,075)$ & $(-4,352 ;-3,130)$ & $(-0,018 ; 0,765)$ \\
\hline
\end{tabular}

Tabela 2.4: Média a posteriori, [desvio padrão] e (HDP 95\%), estimação conjunta.

\begin{tabular}{ccccc}
\hline cópula & $r_{1}$ & $r_{2}$ & $\alpha$ & $\tau_{\alpha}$ \\
\hline Estável & $0,782[0,096]$ & $0,818[0,071]$ & $0,792[0,057]$ & $0,208[0,057]$ \\
Positiva & $(0,604 ; 0,968)$ & $(0,681 ; 0,957)$ & $(0,682 ; 0,902)$ & $(0,098 ; 0,319)$ \\
\hline Frank & $0,815[0,100]$ & $0,834[0,073]$ & $0,158[0,103]$ & $0,215[0,061]$ \\
& $(0,632 ; 1,012)$ & $(0,694 ; 0,985)$ & $(0,018 ; 0,350)$ & $(0,102 ; 0,333)$ \\
\hline Clayton & $0,810[0,098]$ & $0,833[0,073]$ & $1,063[0,352]$ & $0,339[0,075]$ \\
& $(0,630 ; 1,011)$ & $(0,695 ; 0,981)$ & $(0,352 ; 1,723)$ & $(0,188 ; 0,483)$ \\
\hline
\end{tabular}


do tau de Kendall obtida da estimação em duas etapas (veja Tabela 2.2) para as três cópulas consideradas é menor que a obtida no procedimento de estimação conjunta, podemos dizer que a estimação da associação é mais eficiente quando as marginais são extraídas e então uma cópula é assumida. A covariável tem efeito diferente dependendo do tratamento, como foi notado previamente estudando as estimativas de Kaplan-Meier. Em particular, se são comparadas ambas estimativas para os efeitos da covariável em relação ao procedimento de estimação, podemos ver que este efeito é subestimado e com uma maior variabilidade quando é assumida independência entre os tempos de falha.

Notamos também que as figuras apresentadas nas tabelas anteriores para a cópula de Clayton são semelhantes às obtidas por Huster et al. (1989) que assumiu que os parâmetros de forma das distribuições Weibull são iguais para ambos tempos, i.e., $r_{1}=r_{2}=r$, usando a cópula de Clayton com função de razões cruzadas $\theta(v)=\alpha, \alpha>1$, considerando a mesma covariável usada nesta seção.

\section{Comparação de Modelos}

Na seção anterior consideramos três modelos de cópula para modelar a dependência entre os tempos de falha. Para ajudar a decidir qual modelo é o mais apropriado para os dados consideramos os seguintes enfoques: um método descritivo baseado na função de razões cruzadas definida em (2.2.2) e estudada por Clayton (1978), um método de seleção de modelos preditivo e o critério BIC. considerados em Sahu e Dey (2000): e a estatística CPO. discutida em Ibrahim et al. (2001). 
Na Seção 2.2 apresentamos a função de razões cruzadas $\theta^{*}\left(t_{1}, t_{2}\right)$ como uma função da função de sobrevivência bivariada para os três modelos de cópula pesquisados. Para a cópula de Clayton esta função é constante e para os outros dois modelos (Estável Positiva e Frank), ela é decrescente em $t_{1}$ e em $t_{2}$. Com o propósito de obter uma idéia melhor da variação de associação ao longo do tempo, examinamos graficamente a função de razões cruzadas a posteriori para os três modelos de cópula e a comparamos com uma alternativa não paramétrica. A função de razões cruzadas a posteriori está baseada na dependência paramétrica obtida do procedimento em duas etapas e marginais dadas pelo estimador de Kaplan-Meier. A estimativa não paramétrica está baseada em uma versão discreta da função de razões cruzadas dada em Oakes (1989).

As Figuras 2.2 a 2.5 apresentam as estimativas da função de razões cruzadas a posteriori para cada cópula e a alternativa não paramétrica de Oakes (1989). As Figuras 2.2 e 2.3 correspondem aos pacientes adultos e as Figuras 2.4 e 2.5 a pacientes jovens. Em cada gráfico são mostradas as estimativas de $\theta^{*}\left(t_{1}, t_{2}\right)$ como uma função de $t_{j}$; para certos valores selecionados de $t_{k}, \operatorname{com} k, j=1,2, k \neq j$.

É possível observar dos gráficos que quando são considerados tempos de ocorrência menores, tanto o grupo de pacientes adultos como o de pacientes jovens, a curva mais próxima à não paramétrica é a do modelo baseado na cópula de Clayton. Se os tempos maiores são considerados (principalmente tempos censurados), observa-se que a cópula de Frank tem um desempenho melhor com relação ao estimador de Oakes. Finalmente, nos tempos restantes. o melhor desempenho é dado pelo estimador obtido com base na 

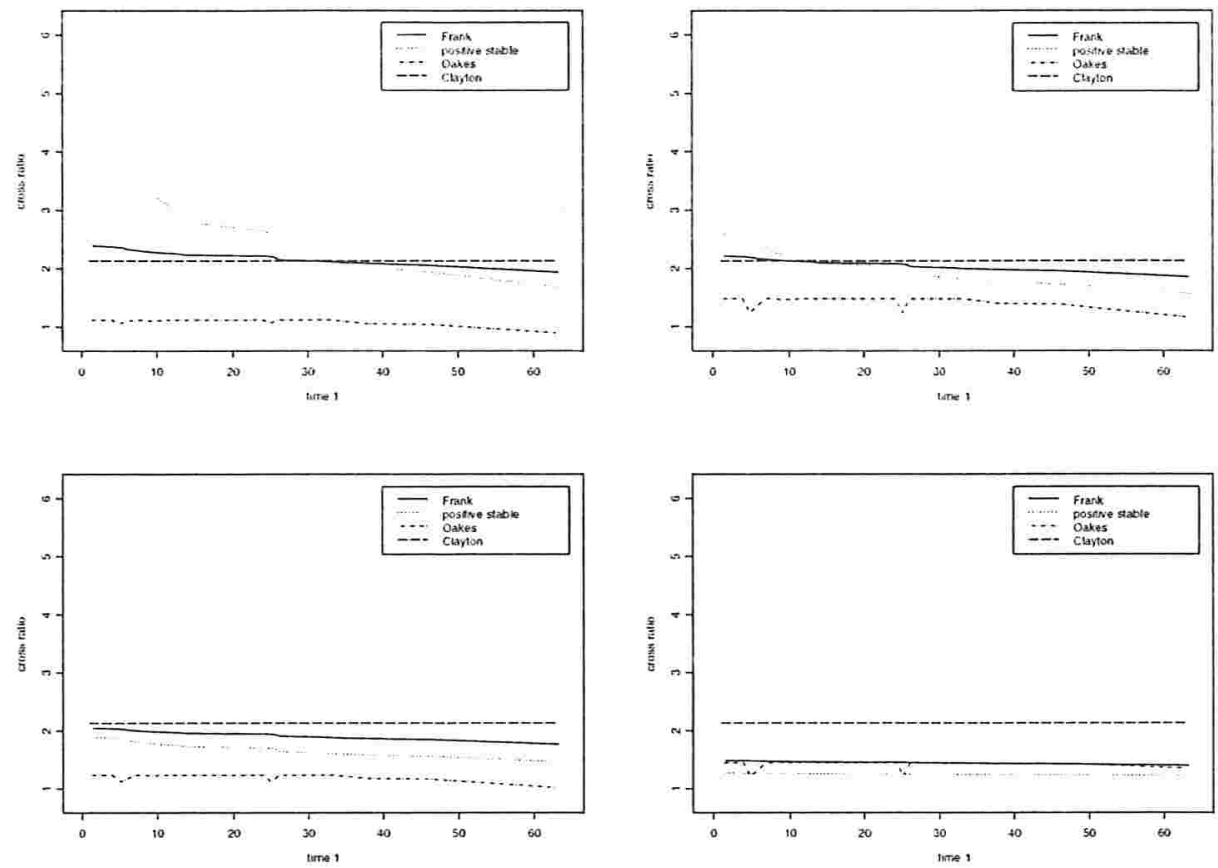

Figura 2.2: Função de razões cruzadas estimada para dados de retinopatia diabética, $t=\left(t_{1}, t_{2}=1\right)$ (esquerda acima), $t=\left(t_{1}, t_{2}=6\right)$ (direita acima), $t=\left(t_{1}, t_{2}=12\right)$ (esquerda embaixo) e $t=\left(t_{1}, t_{2}=48\right)$ (direita embaixo), pacientes adultos.

cópula Estável Positiva.

Note que estamos implicitamente assumindo que o estimador não paramétrico de Oakes representa melhor os dados (embora existam observações censuradas): no sentido que ele não é perturbado por formas paramétricas.

O enfoque preditivo de seleção de modelos é baseado em um critério proposto por Gelfand (1996). e generalizado por Gelfand e Ghosh (1998) para dados 

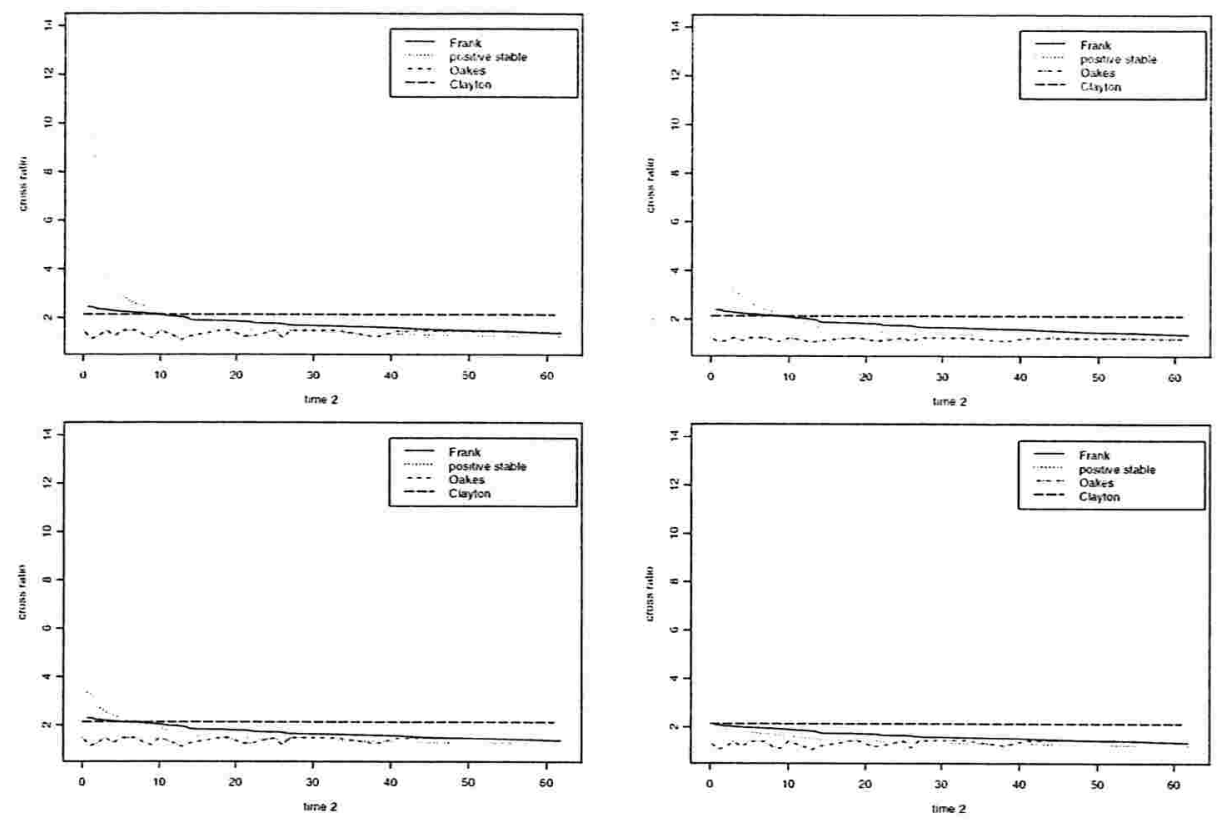

Figura 2.3: Função de razões cruzadas estimada para dados de retinopatia diabética, $t=\left(t_{1}=1, t_{2}\right)$ (esquerda acima), $t=\left(t_{1}=6, t_{2}\right)$ (direita acima), $t=\left(t_{1}=12, t_{2}\right)$ (esquerda embaixo) e $t=\left(t_{1}=48, t_{2}\right)$ (direita embaixo), pacientes adultos.

censurados. Este critério é determinado por

$$
D=\sum_{i=1}^{n} \operatorname{tr}\left(\boldsymbol{\Sigma}_{i}\right)+\sum_{i=1}^{n}\left(\boldsymbol{\mu}_{i}-\boldsymbol{v}_{i}\right)^{T}\left(\boldsymbol{\mu}_{i}-\boldsymbol{v}_{i}\right),
$$

em que $\mu_{i}$ e $\Sigma_{i}$ denotam a média e a covariância da distribuição preditiva a posteriori de observações futuras para o modelo considerado e $v_{i}$ corresponde ao tempo de falha se a i-ésima observação é uma falha, ou é o máximo entre o tempo de censura e $\mu_{i}$ : se o $i$-ésimo tempo corresponde a censura. Este critério é obtido com base no valor esperado da distribuição preditiva,

$$
\pi\left(t_{r e p} \mid t_{o b s}\right)=\int \pi\left(t_{r e p} \mid \theta\right) \pi\left(\theta \mid t_{o b s}\right) d \theta \text {. }
$$



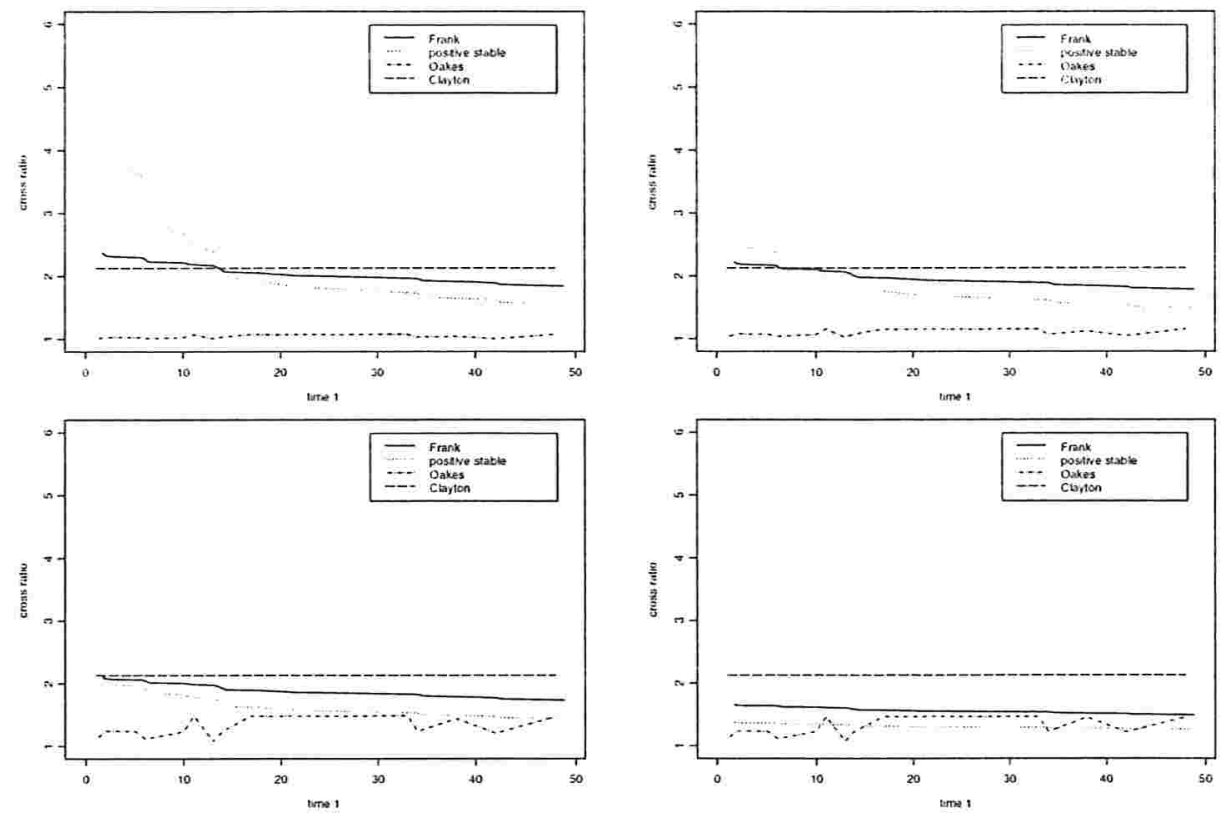

Figura 2.4: Função de razões cruzadas estimada para dados de retinopatia diabética, $t=\left(t_{1}, t_{2}=1\right)$ (esquerda acima), $t=\left(t_{1}, t_{2}=6\right)$ (direita acima), $t=\left(t_{1}, t_{2}=12\right)$ (esquerda embaixo) e $t=\left(t_{1}, t_{2}=48\right)$ (direita embaixo), pacientes jovens.

considerando função de perda quadrática para o erro,

$$
L=\left(t_{r e p}-t_{o b s}\right)^{T}\left(t_{r e p}-t_{o b s}\right),
$$

em que $t_{\text {rep }}$ denota um conjunto de observações futuras para o modelo considerado, e $t_{o b s}$ são os tempos de falha observados.

Conforme Gelfand (1996), para estimar $\mu_{i}$ e $\Sigma_{i}$, primeiro geramos $\theta^{(1)} \ldots, \theta^{(B)}$ da distribuição a posteriori $\pi\left(\theta \mid t_{\text {obs }}\right)$, e en seguida tomamos uma amostra aleatória $t_{i \text { rep }}^{(r)}$ que corresponde a uma amostra da clensidade preditiva. com $1 \leq r \leq B$. 

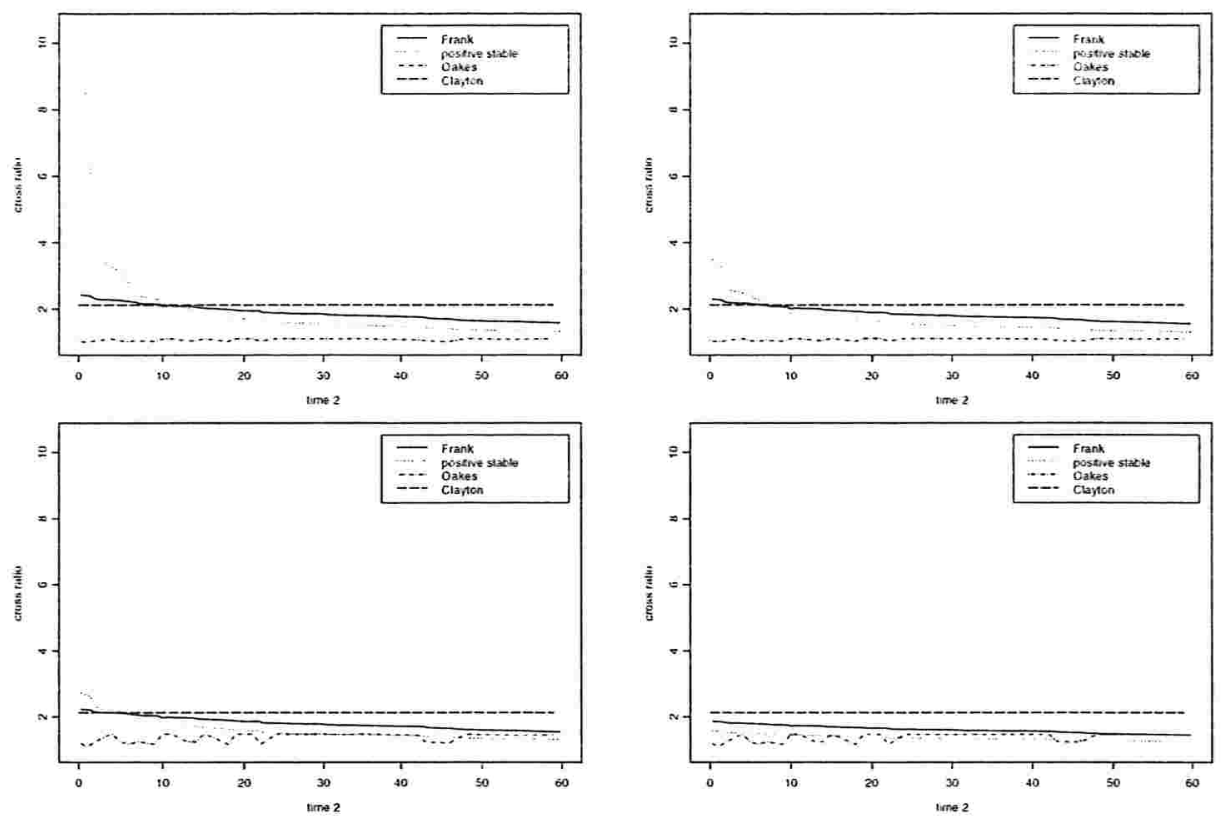

Figura 2.5: Função de razões cruzadas estimada para dados de retinopatia diabética, $t=\left(t_{1}=1, t_{2}\right)$ (esquerda acima), $t=\left(t_{1}=6, t_{2}\right)$ (direita acima), $t=\left(t_{1}=12, t_{2}\right)$ (esquerda embaixo) e $t=\left(t_{1}=48, t_{2}\right)$ (direita embaixo), pacientes jovens.

Para gerar pares aleatórios com estrutura de dependência dada pela cópula Estável Positiva, usamos o método da transformação dado em Lee (1979). Para gerar dados a partir da cópula de Clayton, seguimos o algoritmo dado em Marshall e Olkin (1988). Para a família de Frank, usamos o algoritmo de Genest (1987) para gerar pares aleatórios.

A partir das amostras a posteriori obtidas usando WinBugs 1.4.1, foram desenvolvidos programas no aplicativo SAS 9.0. usando o módulo IML para o cálculo do critério $D$ dado em (2.4.3). 
A estatística CPO (Conditional Predictive Ordinate) para a $i$-ésima observação é definida por

$$
C P O_{i}=f\left(t_{i} \mid D^{(-i)}\right)=\int f\left(t_{i} \mid \theta, x_{i}\right) \pi\left(\theta \mid D^{(-i)}\right) d \theta ;
$$

em que $t_{i}$ denota a variável resposta, $x_{i}$ o vetor de covariáveis para o caso $i$, $D^{(-i)}$ denota os dados sem a $i$-ésima observação e $\pi\left(\theta \mid D^{(-i)}\right)$ é a densidade a posteriori de $\theta$ dado os dados $D^{(-i)}$.

Uma estatística de resumo baseada no $C P O$ é o logaritmo da pseudo verossimilhança marginal LPML (logarithm pseudo marginal likelihood) definida como

$$
L P M L=\sum_{i=1}^{n} \ln \left(C P O_{i}\right) .
$$

A média de $L P M L$ é usada neste trabalho para comparar o desempenho dos modelos, i.e.,

$$
A L P M L=\frac{L P M L}{n},
$$

em que $n$ é o tamanho da amostra. Esta estatística pode ser interpretada como uma pseudo verossimilhança marginal relativa (Ibrahim et al., 2001).

O critério BIC usado em esta seção é o mesmo usado em Sahu e Dey (2000). Como eles comentam, não é possível maximizar a log verosimilhança requerida para calcular o BIC a partir de uma implementação via MCMC. Assim, o valor médio da função de log verosimilhança avaliada em cada iteração MCAIC pode ser usada como uma aproximação. Usamos esta aproximação pois é um método rápido e fácil para comparar diferentes modelos. Este critério pode ser definido como

$$
\widehat{B I C}=-\frac{2}{B} \sum_{k=1}^{B} \sum_{i=1}^{n} \ln L_{i}\left(\theta_{k} \mid z_{i}, \delta_{i}\right)+p \ln n
$$


em que $\theta_{k}$ corresponde a uma amostra da distribuição a posteriori obtida na $k$-ésima iteração em um algoritmo MCMC e $p$ é o número de parâmetros associado ao modelo.

As Tabelas 2.5 e 2.6, contém os valores de (2.4.3), (2.4.4) e (2.4.5) para as cópulas Estável Positiva, Clayton e Frank, considerando os procedimentos de estimação em duas etapas e de estimação conjunta.

A seguinte notação é considerada: EEP e WEP correspondem à cópula Estável Positiva como estrutura de dependência com marginais exponencial e Weibull, respectivamente; analogamente, ECL e WCL correspondem à cópula de Clayton e a cópula de Frank é denotada por EFR e WFR. Também na Tabela 2.5 incluem-se as cópulas Estável Positiva, Clayton e Frank com distribuições marginais estimadas pelo método de Kaplan-Meier, denotadas por KMEP, KMCL e KMFR, respectivamente.

A Tabela 2.5 mostra que os critérios $B I C$ e $A P L M L$ concordam na classificação dos modelos, com o modelo KMFR apresentando o melhor ajuste aos dados. Note que neste caso, o número de parâmetros não tem efeito no critério $B I C$. O critério preditivo $D$ sugiere que um melhor ajuste é dado pelos modelos com marginais exponencial que com marginais Weibull. Note também que os três critérios apresentam valores próximos para os modelos Frank e Clayton.

Na Figura 2.6 apresentamos o gráfico da diferença entre $\log (C P O$. ECL) e $\log (C P O . \mathrm{EFR})$ versus o número das observações. Observa-se que aproximadamente o $58 \%$ das observações estão melhor ajustadas pelo modelo CL. O pseudo fator de Bayes comparando os modelos EFR e ECL é igual a 1.604, indicando que não é possível discriminar entre os dois modelos. 
Tabela 2.5: Critérios de seleção para os dados de retinopatia, estimação em 2 etapas.

\begin{tabular}{cccc}
\hline Modelo & $D$ & $B I C$ & APLML \\
\hline WFR & 1521,20 & 212,24 & $-0,5280$ \\
WCL & 1522,48 & 212,77 & $-0,5296$ \\
EFR & 1157,21 & 219,00 & $-0,5452$ \\
ECL & 1155,66 & 219,92 & $-0,5476$ \\
EEP & 1161,77 & 374,90 & $-0,9411$ \\
WEP & 1524,60 & 379,72 & $-0,9532$ \\
KMCL & -- & 212,53 & $-0,5289$ \\
KMFR & -- & 211,94 & $-0,5271$ \\
KMEP & -- & 214,26 & $-0,5331$ \\
\hline
\end{tabular}

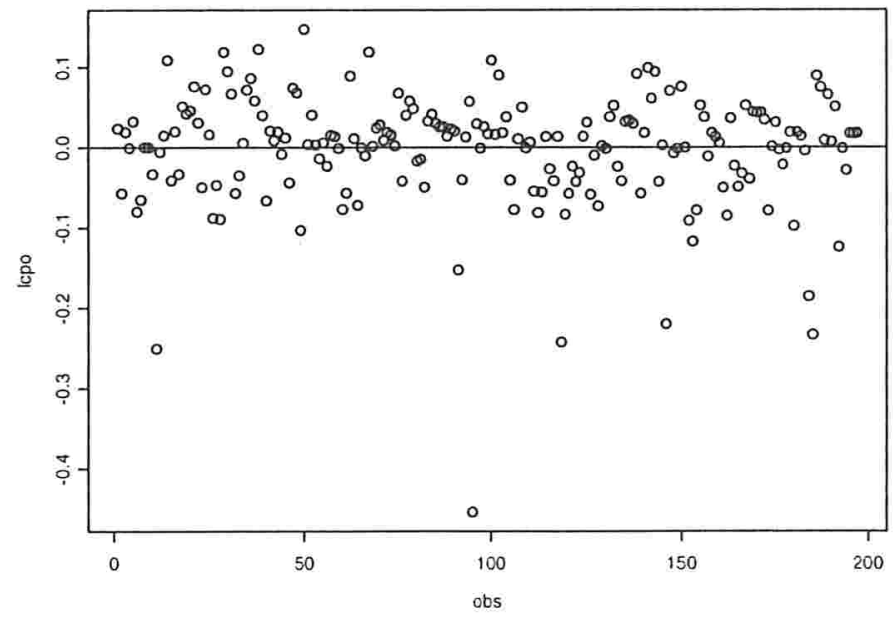

Figura 2.6: Logaritmo da razão do CPO: Modelos ECL versus EFR para os dados de retinopatia. estimação em 2 etapas. 
Tabela 2.6: Critérios de seleção para os dados de retinopatia, estimação conjunta.

\begin{tabular}{cccc}
\hline Modelo & $D$ & $B I C$ & $A P L M L$ \\
\hline ECL & 1171,02 & 1308,02 & $-3,2660$ \\
EFR & 1173,04 & 1689,36 & $-4,2348$ \\
EEP & 1181,32 & 1691,64 & $-4,2405$ \\
WFR & 1520,38 & 1694,03 & $-4,2223$ \\
WCL & 1559,80 & 1694,61 & $-4,2248$ \\
WEP & 1604,11 & 1695,03 & $-4,2256$ \\
\hline
\end{tabular}

Da Tabela 2.6 podemos notar que o modelo ECL tem o melhor ajuste segundo os três critérios considerados. Neste caso de estimação conjunta, os critérios preditivo $D$ e $B I C$ apresentam-se coincidentes com respeito à seleção de modelos. Notamos também que quando consideramos marginais exponencial, temos um melhor desempenho comparado aos modelos com marginais Weibull, tanto para o critério preditivo como para o $B I C$. Em particular, o critério $B I C$ penaliza pelo número de parâmetros neste caso.

Este conjunto de dados também foi estudado por Sahu e Dey (2000) considerando um modelo exponencial bivariado (EMO) e um modelo Weibull bivariado (WMO). Os correspondentes valores de $D$ e $B I C$ foram 1174,22 e 1692.96 para o modelo EMIO, e 1584.72 e 1703.05 para o modelo WMO. Com base em isto, podemos concluir que os modelos ECL e EFR apresentam uma melhor performance que os modelos consiclerados por Sahu e Dey (2000). 
Concluimos esta aplicação destacando que, em todos os casos, a cópula Estável Positiva teve o desempenho menos favorável. Embora os modelos baseados nas cópulas de Clayton e Frank tiveram valores próximos com respeito aos critérios de seleção usados, o modelo de Clayton é mais fácil de implementar computacionalmente e teve função de razões cruzadas mais perto da alternativa não paramétrica de Oakes, ou seja, entre os modelos considerados, o modelo de Clayton com marginais Kaplan-Meier é a melhor escolha para este conjunto de dados.

\subsubsection{Duração da Remissão de Pacientes com Leucemia}

Um experimento clínico envolvendo 62 crianças com leucemia aguda foi realizado em 11 hospitais norte-americanos. Os pacientes receberam um tratamento inicial com prednisona e foram classificados segundo a intensidade da remissão da doença (parcial ou completa). Em seguida, foram aleatorizados em um de dois grupos de tratamento a uma terapia de apoio à remissão do seguinte modo: Em cada hospital os pacientes foram pareados de acordo com o estado da remissão (parcial ou completa) e em cada par um paciente foi escolhido aleatoriamente e recebeu a droga 6-mercaptopurine (6-MIP), ao passo que o outro paciente recebeu placebo. O dados consistem na duração da remissão, i.e., o intervalo de tempo desde a aleatorização até o tempo de recaída, sendo medidos 21 pares completos de pacientes. $\mathrm{O}$ tempo (em semanas) de remissão da recidiva é a resposta de interesse. Os pacientes foram censurados ao final do estudo. 
Os dados foram apresentados primeiro por Freireich et al. (1963) e desde então têm sido frequentemente usados na literatura de dados de sobrevivência, sendo que a maioria das ilustrações negligenciam o emparelhamento. Veja em Andersen et al. (1993) uma lista de publicações com esta consideração.

A percentagem de censura para o tempo de remissão de placebo $\left(T_{1}\right)$ foi de $0 \%$, e para o tempo de remissão 6-MP $\left(T_{2}\right)$ foi de $57,1 \%$. Além do tratamento, a única variável conhecida é o estado de remissão (Status=0 : remissão parcial, Status=1 : remissão completa). Em 23,8\% dos pares ocorre remissão parcial e o complemento, $76,2 \%$, remissão completa.

Dentro de uma perspectiva clássica, estes dados foram analizados também através de um modelo de fragilidade gama por Andersen et al. (1993).

A Figura 2.7 apresenta as curvas de sobrevivência de Kaplan-Meier para cada tempo de remissão, separados por status. Observa-se que, aparentemente, o tratamento é mais efetivo para pacientes com remissão parcial.

Note também que para os pacientes que receberam a droga, as curvas de Kaplan-Meier sugerem a presença de uma proporção de individuos curados, desde que os tempos maiores são censurados e as funções de sobrevivência de Kaplan-.Meier são impropias.

Para realizar a estimação paramétrica em duas etapas considere $T_{1} \sim$ Teibull $\left(r, \lambda_{1}\right)$ e $T_{2} \sim \exp \left(\lambda_{2}\right)$. Ao explorar o efeito da covariável nos dois tempos, resultou que a covariável não é significativa. Assim. considere $\lambda_{j}=\exp \left(.3_{0 j}\right)$. 
Figura 2.7: Função de sobrevivência Kaplan-Meier dados de leucemia.
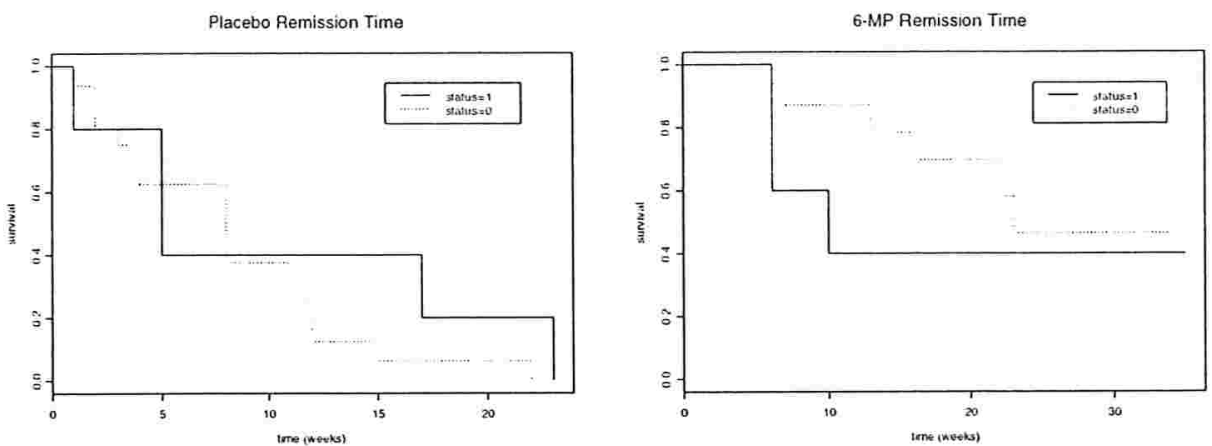

Como distribuições a priori para os parâmetros da distribuição Weibull consideramos $r \sim \exp (0,001), \beta_{01} \sim \operatorname{Normal}\left(0,100^{2}\right)$ e para os parâmetros da distribuição exponencial $\beta_{02} \sim \operatorname{Normal}\left(0,100^{2}\right)$; isto é, distribuições a priori difusas ou vagas. Conforme já mencionado, a distribuição a posteriori (2.3.2), é log-côncava para as prioris consideradas e o algoritmo de rejeição adaptativo é usado para amostrar da distribuição a posteriori no programa WinBugs 1.4.1. Depois de um burn-in de 1.000 valores, os resultados da estimação para cada tempo foram obtidos e são apresentados na Tabela 2.7, considerando $n=2.000$ valores amostrados.

Tabela 2.7: Média a posteriori, [desvio padrão] e (HDP 95\%).

\begin{tabular}{ccc}
\hline$r$ & $3_{01}$ & $3_{02}$ \\
\hline $1,400[0,244]$ & $-3,186[0,669]$ & $-3,752[0,350]$ \\
$(0,972 ; 1,900)$ & $(-4,544:-1.983)$ & $(-4,413:-3.062)$ \\
\hline
\end{tabular}


Da Tabela 2.7 é possível dizer que tanto o modelo Weibull como o modelo exponencial, se ajustam aos dados. Embora o intervalo de credibilidade HDP para o parâmetro de forma da Weibull contenha o valor 1, a Figura 2.8 apresentada adiante mostra que o valor 1 encontra-se bem na cauda da densidade a posteriori.

Seguindo com a etapa 2 da estimação, considere a cópula de Frank dada em (2.2.8) como estrutura de dependência para ser usada na pseudoverossimilhança em (2.3.4), fornecendo a "distribuição a posteriori" (2.3.3), com distribuição a priori $\gamma \sim \operatorname{Normal}\left(a, b^{2}\right)$. As funções de sobrevivência marginais usadas em (2.3.4) foram Weibull com estimativas $\widetilde{r}$ e $\widetilde{\lambda}_{1}=\exp \left(\widetilde{\beta}_{01}\right)$ para $T_{1}$, e exponencial com estimativa $\widetilde{\lambda}_{2}=\exp \left(\widetilde{\beta}_{02}\right)$ para $T_{2}$, em que $\left(\widetilde{\boldsymbol{\theta}}_{1}, \widetilde{\theta}_{2}\right)=\left(\widetilde{r}, \widetilde{\beta}_{01} ; \widetilde{\beta}_{02}\right)$ são dadas pelas correspondentes médias a posteriori, isto é, $(1,400 ;-3,186 ;-3,752)$.

Dadas as especificações anteriores a pseudo-verossimilhança em (2.3.4) pode ser escrita como

$$
\begin{aligned}
& L\left(\gamma \mid z_{1}, \boldsymbol{z}_{2}, \boldsymbol{\delta}_{1}, \boldsymbol{\delta}_{2}, \widetilde{\boldsymbol{\theta}}_{1}, \widetilde{\theta}_{2}\right)=\prod_{i=1}^{n}(-\gamma)^{\delta_{i 1} \delta_{i 2}} e^{-\gamma\left(\delta_{i 1} e^{-\widetilde{\lambda_{1}} z_{i 1} \tilde{\tau}}+\delta_{i 2} e^{-\widetilde{\lambda_{2}} z_{i 2}}\right)}\left(e^{-\gamma}-1\right)^{\delta_{i 1} \delta_{i 2}} \\
& \cdot\left(e^{-\gamma e^{-\widetilde{\lambda_{2}} z_{i 2}}}-1\right)^{\delta_{i 1}\left(1-\delta_{i 2}\right)}\left(e^{-\gamma e^{-\widetilde{\lambda_{1}} z_{i 1} \tilde{r}}}-1\right)^{\delta_{i 2}\left(1-\delta_{i 1}\right)} \\
& \cdot r_{\gamma i}^{-\left(\delta_{i 1}+\delta_{i 2}\right)}\left(-\gamma^{-1} \ln \frac{r_{\gamma i}}{e^{-\gamma}-1}\right)^{\left(1-\delta_{i 1}\right)\left(1-\delta_{i 2}\right)}
\end{aligned}
$$

em que $r_{\gamma i}=e^{-\gamma}-1+\left(e^{-\gamma e^{-\widetilde{\lambda_{1}} z_{i 1} \tilde{r}}}-1\right)\left(e^{-\gamma e^{-\widetilde{\lambda_{2}} z_{i 2}}}-1\right)$.

Logo: a "distribuição a posteriori" para o parâmetro de dependência é dada por 


$$
\begin{aligned}
& \pi\left(\gamma \mid z_{1}, z_{2}, \delta_{1}, \delta_{2}, \widetilde{\theta}_{1}, \widetilde{\theta}_{2}\right) \propto(-\gamma)^{\sum_{i} \delta_{i 1} \delta_{i 2}} e^{-\gamma\left(\sum_{i}\left(\delta_{i 1} e^{-\widetilde{\lambda_{1}} z_{i 1}{ }^{r}}+\tilde{\delta}_{i 2} e^{-\widetilde{\lambda_{2}} z_{i 2}}\right)-a / b^{2}\right)} \\
& \cdot e^{-\frac{\gamma^{2}}{2 b^{2}}}\left(e^{-\gamma}-1\right)^{\sum_{i} \delta_{i 1} \delta_{i 2}} \prod_{i=1}^{n} r_{\gamma i}^{-\left(\delta_{i 1}+\delta_{i 2}\right)} \\
& \cdot\left(e^{-\gamma e^{-\widetilde{\lambda_{2}} z_{i 2}}}-1\right)^{\delta_{i 1}\left(1-\delta_{i 2}\right)}\left(e^{-\gamma e^{-\widetilde{\lambda_{1}} z_{i 1} \tilde{r}}}-1\right)^{\delta_{i 2}\left(1-\delta_{i 1}\right)} \\
& \cdot\left(-\gamma^{-1} \ln \frac{r_{\gamma_{i}}}{e^{-\gamma}-1}\right)^{\left(1-\delta_{i 1}\right)\left(1-\dot{\delta}_{i 2}\right)}
\end{aligned}
$$

Implementando esta última expressão no WinBugs 1.4.1, com distribuição a priori $\gamma \sim \operatorname{Normal}\left(0,100^{2}\right)$, o algoritmo de Metropolis-Hastings foi usado para amostrar valores desta distribuição a posteriori. Depois de um burn-in de 4.000 valores e uma amostra de 2.000 dados, obteve-se os resultados na Tabela 2.8 .

Tabela 2.8: Média a posteriori, [desvio padrão] e (HDP 95\%).

\begin{tabular}{cc}
$\gamma$ & $\tau_{\gamma}$ \\
\hline$-0,929[1,450]$ & $-0,125[0,162]$ \\
$(-3,813 ; 1,869)$ & $(-0,407 ; 0,216)$ \\
\hline
\end{tabular}

Da tabela observa-se que existe uma associação negativa entre os tempos de remissão, mas ela não é significativa (veja a densidade a posteriori para o tau de Kendall na Figura 2.8).

Estes resultados concordam com Hougaard (2000): que através de um estimador não paramétrico para o tau de Kendall. obteve uma estimatira de -0.110 0.170], e de -0.090 [0.180] quando esse estimador é ajustado 
considerando censura.

Dentro de uma perspectiva clássica, Andersen et al. (1993) ajusta um modelo de fragilidade gama a esses dados. Eles concluem também que o parâmetro de fragilidade, a variância da distribuição gama, não é significativamente diferente de zero.

Figura 2.8: Função de densidade a posteriori, parâmetros $r$ e $\tau_{\gamma}$, dados de leucemia.
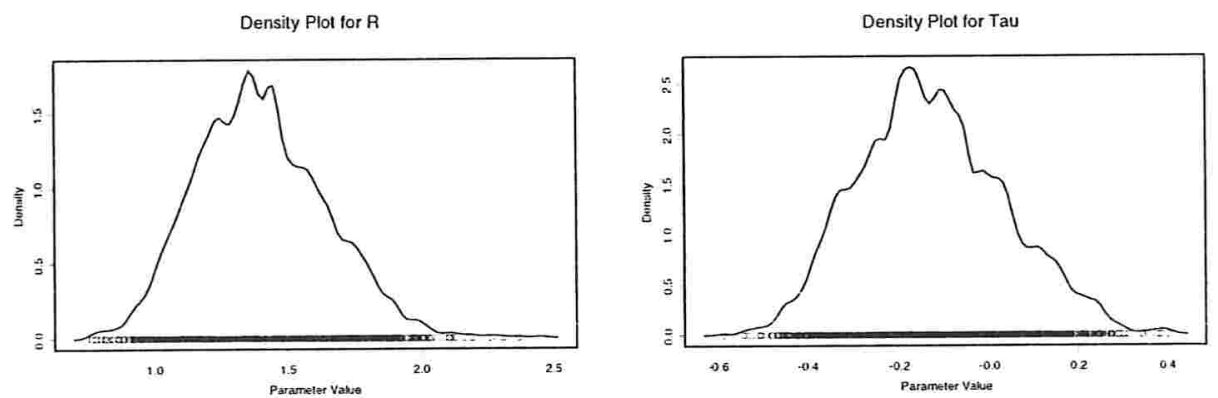


\section{Capítulo 3}

\section{Teorema Central do Limite Funcional para Cópulas em Análise de Sobrevivência}

\subsection{Introdução}

O objetivo deste capítulo é estudar as propriedades assintóticas do estimador da função de sobrevivência bivariada $C_{\alpha}\left(S_{1}, S_{2}\right)$, utilizando a relação funcional entre as funções de sobrevivência marginal $\left(S_{1}, S_{2}\right)$ e uma particular cópula $C_{\alpha}$.

Especificamente, estudamos a consistência e a convergência fraca do estimador paramétrico $C_{\widehat{\alpha}_{n}}\left(\widehat{S}_{1 n}, \widehat{S}_{2 n}\right)$, obtido com base em uma análise Bayesiana, sob função de perda quadrática e um procedimento de estimação em duas etapas.

As soluções obtidas nesta seção derivam-se de uma decomposição algébrica 
da função de sobrevivência bivariada $C_{\alpha}\left(S_{1}, S_{2}\right)$ em quantidades que podem ser estudadas separadamente. Em particular utilizam-se resultados referentes à convergência quase certa e em distribuição de estimadores obtidos considerando métodos de estimação via MCMC, convergência quase certa de funções eqüicontínuas e uniformemente contínuas, e o método delta para funcionais. Alguns conceitos e resultados utilizados aparecem descritos nos Apêndices A e B.

No que segue estaremos usando a seguinte notação.

Considere $\left(T_{1}, T_{2}\right)$ variáveis aleatórias contínuas não negativas com função de sobrevivência $\left(S_{1}, S_{2}\right)$, respectivamente, e com função de sobrevivência conjunta dada por

$$
S\left(t_{1}, t_{2}\right)=C_{\alpha}\left(S_{1}\left(t_{1}\right), S_{2}\left(t_{2}\right)\right),
$$

sujeita a $S\left(t_{1}, 0\right)=S_{1}\left(t_{1}\right), S\left(0, t_{2}\right)=S_{2}\left(t_{2}\right)$.

- $C_{\alpha}$, com $\alpha \in \mathcal{A}$, denota a cópula associada a $S$, para todo $t_{1} \in t_{S_{1}}$, e $t_{2} \in t_{S_{2}}$, em que $t_{S_{j}}=\left[0, \tau_{j}\right] \operatorname{com} \tau_{j}=\sup \left\{t_{j}: S_{j}\left(t_{j}\right)>0\right\}, j=1,2$.

- Para $i=1, \ldots, n$, sejam $\left(T_{i 1}, T_{i 2}\right)$ uma amostra aleatória de $\left(T_{1}, T_{2}\right)$ definidas no espaço de probabilidade $\left(\Omega, \mathcal{B}, \mathcal{P}_{T_{1}, T_{2}}\right)$, em que $\Omega=\mathbb{R}^{+} \times$ $\mathbb{R}^{+}: \mathcal{B}=\mathcal{B}_{n} \times \mathcal{B}_{n}$, e $\mathcal{P}_{T_{1}, T_{2}}=\left\{P_{\alpha,\left(T_{1}, T_{2}\right)} ; \alpha \in \mathcal{A}\right\}$. O estimador da função de sobrevivência bivariada dado em (3.1.1) pode ser escrito como

$$
\widehat{S}\left(t_{1}, t_{2}\right)=C_{\widehat{\alpha}_{n}}\left(\widehat{S}_{1 n}\left(t_{1}\right), \widehat{S}_{2 n}\left(t_{2}\right)\right)
$$

- $\widehat{\alpha}_{n}$ é o estimador Bayesiano do parâmetro de dependéncia, obtido sob função de perda quadrática e considerando um procedimento de estimação em duas etapas e distribuição a priori $\bar{\pi}(\alpha)$ contínua: e 
- $\widehat{S}_{j n}$ é o estimador Bayesiano paramétrico, sob função de perda quadrática da função de sobrevivência marginal $S_{j}$, usado na etapa 1 do procedimento de estimação em duas etapas, $j=1,2$.

Note que implicitamente estamos denotando $\widehat{S}_{j n}(\cdot, \widehat{\theta})$ e $S_{j}(\cdot, \theta)$ por $\widehat{S}_{j n}(\cdot)$ e $S_{j}(\cdot)$, respectivamente, com $\widehat{\theta}$ sendo um parâmetro ou vetor de parâmetros estimado na etapa 1 do procedimento de estimação.

\section{Observação 3.1 .1}

Estes estimadores são obtidos considerando métodos de estimação via MCMC.

Sem perda de generalidade, denotando a distribuição a posteriori por $\pi(\theta \mid X)$ e pelo vetor $\left(\theta_{1}^{(k)}, \ldots, \theta_{d}^{(k)}\right)$ as realizações de uma cadeia de Markov na k-ésima iteração em um algoritmo do amostrador de Gibbs, Tanner (1996), Capítulo 6, resume os seguintes resultados para qualquer função integrável $f:$

o a distribuição conjunta de $\left(\theta_{1}^{(k)}, \ldots, \theta_{d}^{(k)}\right)$ converge geometricamente a $\pi(\boldsymbol{\theta} \mid X)$ quando $k \rightarrow \infty$,

$\circ n^{-1} \sum_{k=1}^{n} f\left(\boldsymbol{\theta}^{(k)}\right) \stackrel{\text { q.c. }}{\longrightarrow} \int f(\boldsymbol{\theta}) \pi(\boldsymbol{\theta} \mid X) d \boldsymbol{\theta}$, quando $n \rightarrow \infty$,

$\circ n^{1 / 2}\left[n^{-1} \sum_{k=1}^{n} f\left(\boldsymbol{\theta}^{(k)}\right)-\int f(\boldsymbol{\theta}) \pi(\boldsymbol{\theta} \mid X) d \boldsymbol{\theta}\right] \stackrel{D}{\longrightarrow} N\left(0, \sigma_{f}^{2}\right)$

quando $n \rightarrow \infty$.

Chen e Schmeiser (1993) e Geyer (1992) discutem a estimação de $\sigma_{f}^{2}$. 


\subsection{Propriedades assintóticas do estimador $\widehat{S}$}

Nesta seção estudamos consistência e convergência fraca do estimador paramétrico da função de sobrevivência bivariada $S\left(t_{1}, t_{2}\right)=C_{\alpha}\left(S_{1}\left(t_{1}\right), S_{2}\left(t_{2}\right)\right)$. A seguir apresentamos uma decomposição algébrica adequada da função de sobrevivência bivariada.

Dado que desejamos estudar a convergência de $\widehat{S}\left(t_{1}, t_{2}\right)$ para $S\left(t_{1}, t_{2}\right)$, em termos de cópula e funções de sobrevivência marginal, precisamos estudar a convergência de $C_{\widehat{\alpha}_{n}}\left(\widehat{S}_{1 n}\left(t_{1}\right), \widehat{S}_{2 n}\left(t_{2}\right)\right)$ para $C_{\alpha}\left(S_{1}\left(t_{1}\right), S_{2}\left(t_{2}\right)\right)$, quando $n \rightarrow$ $\infty$, para todo $t_{1} \in t_{S_{1}}, t_{2} \in t_{S_{2}}$, e $\alpha \in \mathcal{A}$. Uma forma adequada de escrever esta última expressão é

$$
\begin{array}{r}
n^{1 / 2}\left[C_{\widehat{\alpha}_{n}}\left(\widehat{S}_{1 n}\left(t_{1}\right), \widehat{S}_{2 n}\left(t_{2}\right)\right)-C_{\alpha}\left(S_{1}\left(t_{1}\right), S_{2}\left(t_{2}\right)\right)\right]=n^{1 / 2}\left(\widehat{\alpha}_{n}-\alpha\right) \\
\times \frac{C_{\widehat{\alpha}_{n}}\left(\widehat{S}_{1 n}\left(t_{1}\right), \widehat{S}_{2 n}\left(t_{2}\right)\right)-C_{\alpha}\left(\widehat{S}_{1 n}\left(t_{1}\right), \widehat{S}_{2 n}\left(t_{2}\right)\right)}{\widehat{\alpha}_{n}-\alpha} \\
+n^{1 / 2}\left[C_{\alpha}\left(\widehat{S}_{1 n}\left(t_{1}\right), \widehat{S}_{2 n}\left(t_{2}\right)\right)-C_{\alpha}\left(S_{1}\left(t_{1}\right), S_{2}\left(t_{2}\right)\right)\right],
\end{array}
$$

para todo $t_{1} \in t_{S_{1}}, t_{2} \in t_{S_{2}}$, e $\alpha \in \mathcal{A}$.

Destacamos que a decomposição anterior sugere ver a cópula como um funcional de funções de sobrevivência marginal, e também como uma função do parâmetro de dependência $\alpha \in \mathcal{A}$, para escolhas particulares de uma cópula (e.g. Clayton, Estável Positiva e Frank).

Do primeiro termo à direita em (3.2.1), i.e., $n^{1 / 2}\left(\widehat{\alpha}_{n}-\alpha\right)$, podemos dizer a 
partir de (3.1.3) e (3.1.4) na Observação 3.1.1, que quando $n \rightarrow \infty$.

$$
\begin{gathered}
\widehat{\alpha}_{n}=n^{-1} \sum_{j=1}^{n} \alpha^{(j)} \stackrel{q . c .}{\longrightarrow} \alpha, \\
n^{1 / 2}\left(\widehat{\alpha}_{n}-\alpha\right) \stackrel{D}{\longrightarrow} N\left(0, \sigma_{\alpha}^{2}\right),
\end{gathered}
$$

em que $\sigma_{\alpha}^{2}=\lim _{n \rightarrow \infty} \operatorname{Var}\left[\widehat{\alpha}_{n}\right]$.

O segundo termo à direita em (3.2.1), i.e.,

$$
\frac{C_{\widehat{\alpha}_{n}}\left(\widehat{S}_{1 n}\left(t_{1}\right), \widehat{S}_{2 n}\left(t_{2}\right)\right)-C_{\alpha}\left(\widehat{S}_{1 n}\left(t_{1}\right), \widehat{S}_{2 n}\left(t_{2}\right)\right)}{\widehat{\alpha}_{n}-\alpha},
$$

pode ser visto como uma função $C_{(\cdot)}$ do parâmetro de dependência $\alpha \in \mathcal{A}$, em que as funções de sobrevivência marginal $\left(\widehat{S}_{1 n}\left(t_{1}\right), \widehat{S}_{2 n}\left(t_{2}\right)\right)$ estão fixadas para cada $t_{j} \in t_{S_{j}}, j=1,2$. Assim, o teorema do valor médio pode ser aplicado, conforme discutido a seguir.

Assuma $C_{(\cdot)}: \mathcal{A} \mapsto \mathbb{R}, \mathcal{A} \subset \mathbb{R}$ ser uma função contínua diferenciável, e $\alpha, \widehat{\alpha}_{n} \in \mathcal{A}$ tal que $\left[\alpha, \widehat{\alpha}_{n}\right] \subset \mathcal{A}$. Então, existe $\alpha_{n}^{*} \in \mathcal{A}$ entre $\alpha$ e $\widehat{\alpha}_{n}$ tal que

$$
\frac{C_{\widehat{\alpha}_{n}}\left(\widehat{S}_{1 n}\left(t_{1}\right), \widehat{S}_{2 n}\left(t_{2}\right)\right)-C_{\alpha}\left(\widehat{S}_{1 n}\left(t_{1}\right), \widehat{S}_{2 n}\left(t_{2}\right)\right)}{\widehat{\alpha}_{n}-\alpha}=\dot{C}_{\alpha_{n}^{*}}\left(\widehat{S}_{1 n}\left(t_{1}\right), \widehat{S}_{2 n}\left(t_{2}\right)\right),
$$

em que $\dot{C}_{(\cdot)}$ denota a derivada de $C_{(\cdot)}$ respeito a $\alpha \in \mathcal{A}$, com os argumentos $u$ e $v$ da cópula fixados.

Finalmente, note que o terceiro termo à direita em (3.2.1), i.e.,

$$
n^{1 / 2}\left[C_{\alpha}\left(\widehat{S}_{1 n}\left(t_{1}\right), \widehat{S}_{2 n}\left(t_{2}\right)\right)-C_{\alpha}\left(S_{1}\left(t_{1}\right) \cdot S_{2}\left(t_{2}\right)\right)\right] .
$$

com $a \in \mathcal{A}$ fixo pode ser visto como a diferença de um funcional. digamos $C_{o}^{\prime}$, araliado em $\left(\widehat{S}_{1 n}(\cdot) . \widehat{S}_{2 n}(\cdot)\right)$ e $\left(S_{1}(\cdot) . S_{2}(\cdot)\right)$, para todo $t_{j} \in t_{S_{j}} \cdot j=1.2$. 
Seja $D[0, \infty]$ o espaço das funções contínuas pela esquerda e tendo limites pela direita, caglad, sobre o intervalo $[0, \infty]$ munido com a norma do supremo $\|z\|=\sup _{t}|z(t)|$, com $t \in[0, \infty]$. Definindo $\mathcal{S}$ como o espaço de todas as funções de sobrevivência, em que $\mathcal{S} \subset D[0, \infty]$, o funcional $C_{o}: D[0, \infty] \times$ $D[0, \infty] \mapsto[0,1]$, pode ser definido por

$$
\begin{aligned}
C_{o}\left(H_{1}(\cdot), H_{2}(\cdot) ; t\right) & =C_{\alpha}\left(H_{1}\left(t_{1}\right), H_{2}\left(t_{2}\right)\right) \\
& =\int_{\left\{t_{1}: 0<u \leq H_{1}\left(t_{1}\right)\right\}} \int_{\left\{t_{2}: 0<v \leq H_{2}\left(t_{2}\right)\right\}} d C_{\alpha}(u, v),
\end{aligned}
$$

com $t=\left(t_{1}, t_{2}\right)$, para todo $t_{j} \in t_{S_{j}}, j=1,2$ e $H_{1}, H_{2} \in \mathcal{S}$.

$\mathrm{Na}$ próxima seção estabelecemos a consistência forte para $\dot{C}_{\alpha_{n}^{*}}$, obtido em (3.2.4), para os modelos de cópulas de Clayton, Estável Positiva e Frank. Posteriormente, na seção seguinte, propriedades assintóticas do funcional $C_{\boldsymbol{o}}$, definido em (3.2.5) são estabelecidas. Finalmente; a consistência forte e a convergência fraca para $\widehat{S}$ são demonstradas.

\section{Observação 3.2 .1}

(a) Para os três modelos de cópulas considerados, Clayton, Estável Positiva e Frank, temos de (3.2.2) que $\widehat{\alpha}_{n} \stackrel{\text { q.c. }}{\longrightarrow} \alpha$; quando $n \rightarrow \infty$. Sabemos de (3.2.4) que $\alpha_{n}^{*}$ é uma quantidade entre $\alpha$ e $\widehat{\alpha}_{n}$, e portanto $\left|\alpha_{n}^{*}-\alpha\right|<$ $\left|\widehat{\alpha}_{n}-\alpha\right|$ : implicando que $\alpha_{n}^{*} \stackrel{\text { q.e. }}{\longrightarrow} \alpha$, quando $n \rightarrow \infty$, i.e., o evento $B=\left\{\omega \in \Omega: \alpha_{n}^{*}(\omega) \rightarrow a\right\}$ é de probabilidade 1 .

(b) Para funções de sobrevivència $S_{j}(\cdot, \theta), j=1,2$, vindas de famílias paramétricas contínuas, de (3.1.3) e (3.1.4) na Observação 3.1.1 temos 
que, quando $n \rightarrow \infty$,

$$
\begin{array}{r}
\widehat{S}_{j n}\left(t_{j} ; \widehat{\theta}\right)=n^{-1} \sum_{k=1}^{n} S_{j}\left(t_{j} ; \theta^{(k)}\right) \stackrel{q . c .}{\longrightarrow} S_{j}\left(t_{j} ; \theta\right), \quad \mathrm{e} \\
n^{1 / 2}\left[\widehat{S}_{j n}\left(t_{j} ; \widehat{\theta}\right)-S_{j}\left(t_{j} ; \theta\right)\right] \stackrel{D}{\longrightarrow} N\left(0, \sigma_{S_{j}}^{2}\right),
\end{array}
$$

para todo $t_{j} \in t_{S_{j}}, j=1,2$.

Note também que, como feito no capítulo anterior, a estimação de $\theta$ e, consequentemente, de $S_{j}$ é realizada considerando a presença de tempos censurados.

(c) Notamos que para que a expressão em (3.2.3) esteja bem definida e $\left|\dot{C}_{(\cdot)}\right|$ em (3.2.4) seja limitado, trabalhamos considerando $\alpha$ em um subconjunto compacto de $\mathcal{A}$.

\subsubsection{Consistência forte de $\dot{C}_{\alpha_{n}^{*}}$}

Primeiramente consideramos a seguinte proposição, necessária para provar o Lema 3.2.1, que estabelece a eqüicontinuidade da família de funções $\dot{C}_{(\cdot)}(x, y)$ com $x=S_{1}\left(t_{1}\right)$ e $y=S_{2}\left(t_{2}\right)$, para todo $t_{j} \in t_{S_{j}}$, em que $t_{S_{j}}=\left[0, \tau_{j}\right]$ $\operatorname{com} \tau_{j}=\sup \left\{t_{j}: S_{j}\left(t_{j}\right)>0\right\}, j=1,2$, para as cópulas de Clayton, Estável Positiva e Frank. A prova é postergada para o final deste capítulo.

\section{Proposição 3.2 .1}

Defina $E$ como o conjunto de todas as funções $\dot{C}_{(\cdot)}(x, y): \mathcal{A} \mapsto \mathbb{R}, \mathcal{A} \subset \mathbb{R}$, tal que,

$$
E=\left\{\dot{C}_{(\cdot)}(x, y): x \in\left[S_{1}\left(\tau_{1}\right), 1\right] \wedge y \in\left[S_{2}\left(\tau_{2}\right), 1\right]\right\} .
$$

Ent ão, 
(a) para a cópula de Clayton, $C_{\alpha}(x, y)=w_{\alpha}^{-1 / \alpha}$, em que $w_{\alpha}=x^{-\alpha}+y^{-\alpha}-1$, com $\alpha \in \mathcal{A} \equiv \mathbb{R}^{+}$, temos que $\dot{C}_{\left(\alpha_{n}^{*}\right)}(x, y)$, dada por,

$$
\dot{C}_{\left(\alpha_{n}^{*}\right)}(x, y)=w_{\alpha_{n}^{*}}^{-1 / \alpha_{n}^{*}}\left[\alpha_{n}^{*-2} \ln w_{\alpha_{n}^{*}}+\frac{x^{-\alpha_{n}^{*}} \ln x+y^{-\alpha_{n}^{*}} \ln y}{\alpha_{n}^{*} w_{\alpha_{n}^{*}}}\right],
$$

é uma função eqüicontínua em $\alpha$;

(b) para a cópula Estável Positiva, $C_{\alpha}(x, y)=\exp \left\{-s_{\alpha}^{\alpha}\right\}$, em que $s_{\alpha}=$ $(-\ln x)^{1 / \alpha}+(-\ln y)^{1 / \alpha}, \operatorname{com} \alpha \in \mathcal{A} \equiv(0,1)$, temos que $\dot{C}_{\left(\alpha_{n}^{*}\right)}(x, y)$, dada por,

$$
\begin{aligned}
\dot{C}_{\left(\alpha_{n}^{*}\right)}(x, y)= & \exp \left\{-s_{\alpha_{n}^{*}}^{\alpha_{n}^{*}}\right\} \cdot-s_{\alpha_{n}^{*}}^{\alpha_{n}^{*}} \cdot\left\{\frac { \alpha _ { n } ^ { * } } { s _ { \alpha _ { n } ^ { * } } } \left[-\frac{1}{\alpha_{n}^{* 2}}(-\ln x)^{1 / \alpha_{n}^{*}} \ln (-\ln x)\right.\right. \\
& \left.\left.-\frac{1}{\alpha_{n}^{* 2}}(-\ln y)^{1 / \alpha_{n}^{*}} \ln (-\ln y)\right]+\ln s_{\alpha_{n}^{*}}\right\}
\end{aligned}
$$

é uma função eqüicontínua em $\alpha$;

(c) para a cópula de Frank, $C_{\alpha}(x, y)=\log _{\alpha}\left(\frac{r_{\alpha}}{\alpha-1}\right)$, em que $r_{\alpha}=\alpha-1+$ $\left(\alpha^{x}-1\right)\left(\alpha^{y}-1\right)$, com $\alpha \in \mathcal{A} \equiv(0,1)$, temos que $\dot{C}_{\left(\alpha_{n}^{*}\right)}(x, y)$, dada por,

$$
\begin{aligned}
\dot{C}_{\left(\alpha_{n}^{*}\right)}(x, y)= & \frac{\alpha_{n}^{*}-1}{r_{\alpha_{n}^{*}} \ln \alpha_{n}^{*}}\left[\frac{x \alpha_{n}^{* x-1}\left(\alpha_{n}^{* y}-1\right)+y \alpha_{n}^{* y-1}\left(\alpha_{n}^{* x}-1\right)}{\alpha_{n}^{*}-1}\right. \\
& \left.-\frac{\left(\alpha_{n}^{* x}-1\right)\left(\alpha_{n}^{* y}-1\right)}{\left(\alpha_{n}^{*}-1\right)^{2}}\right]-\frac{\ln \left(\frac{r_{\alpha_{n}^{*}}}{\alpha_{n}^{*}-1}\right)}{\alpha_{n}^{*}\left(\ln \alpha_{n}^{*}\right)^{2}}
\end{aligned}
$$

é uma função eqüicontínua em $\alpha$.

\section{Lema 3.2.1}

Seja $\widehat{S}_{1 n}\left(t_{1}\right) \in\left[S_{1}\left(\tau_{1}\right), 1\right]$ e $\widehat{S}_{2 n}\left(t_{2}\right) \in\left[S_{2}\left(\tau_{2}\right), 1\right]$, para todo $t_{j} \in t_{S_{j}} ; j=$ 1.2. Então. para os três modelos de cópulas considerados. Clayton. Estável 
Positiva e Frank,

$$
\dot{C}_{\alpha_{n}}\left(\widehat{S}_{1 n}, \widehat{S}_{2 n}\right)-\dot{C}_{\alpha}\left(\widehat{S}_{1 n}, \widehat{S}_{2 n}\right) \stackrel{q . c .}{\longrightarrow} 0 .
$$

\section{Prova.}

É uma consequência da proposição anterior. De fato, pela definição de eqüicontinuidade, para os três modelos de cópula considerados, para todo $x \in\left[S_{1}\left(\tau_{1}\right), 1\right], y \in\left[S_{2}\left(\tau_{2}\right), 1\right]$, para todo $\varepsilon>0$ e para $n \geq n_{0}(\varepsilon)$,

$$
\left|\dot{C}_{\alpha_{n}^{*}}(x, y)-\dot{C}_{\alpha}(x, y)\right|<\varepsilon \text {. (q.c.) }
$$

Para todo $w \in \Omega$, considerando $\widehat{S}_{1 n}(\cdot, \omega)=x^{\prime}, \widehat{S}_{2 n}(\cdot, \omega)=y^{\prime}$, para algum $x^{\prime} \in\left[S_{1}\left(\tau_{1}\right), 1\right]$ e algum $y^{\prime} \in\left[S_{2}\left(\tau_{2}\right), 1\right]$, segue a eqüicontinuidade de $\dot{C}_{\alpha_{n}^{*}(\omega)}\left(\widehat{S}_{1 n}(\cdot, \omega), \widehat{S}_{2 n}(\cdot, \omega)\right) \in E$ em $\alpha$ e assim, para todo $\omega \in(\Omega \cap B)=B$ com $\operatorname{Pr}\{B\}=1$, para todo $\varepsilon>0$ e $n \geq n_{0}(\varepsilon)$,

$$
\left|\dot{C}_{\alpha_{n}^{*}(\omega)}\left(\widehat{S}_{1 n}(\cdot, \omega), \widehat{S}_{2 n}(\cdot, \omega)\right)-\dot{C}_{\alpha}\left(\widehat{S}_{1 n}(\cdot, \omega), \widehat{S}_{2 n}(\cdot, \omega)\right)\right|<\varepsilon
$$

e portanto,

$$
\dot{C}_{\alpha_{n}^{*}}\left(\widehat{S}_{1 n}, \widehat{S}_{2 n}\right)-\dot{C}_{\alpha}\left(\widehat{S}_{1 n}, \widehat{S}_{2 n}\right) \stackrel{q . c .}{\longrightarrow} 0 .
$$

Na proposição seguinte estabelecemos a continuidade uniforme da função $\dot{C}_{\alpha}(\cdot, \cdot)$, para os três modelos de cópulas estudados. Essa proposição é usada para provar o Lema 3.2.2 que estabelece a convergência quase certa para $\dot{C}_{o}\left(\widehat{S}_{1 n}, \widehat{S}_{2 n}\right)$. A prova é adiada para o final deste capítulo. 


\section{Proposição 3.2 .2}

Para os modelos de cópula de Clayton, Estável Positiva e Frank, a função $\dot{C}_{\alpha}:\left[S_{1}\left(\tau_{1}\right), 1\right] \times\left[S_{2}\left(\tau_{2}\right), 1\right] \mapsto \mathbb{R}$ é uma função uniformemente contínua.

\section{Lema 3.2.2}

Seja $\widehat{S}_{1 n}\left(t_{1}\right) \in\left[S_{1}\left(\tau_{1}\right), 1\right]$ e $\widehat{S}_{2 n}\left(t_{2}\right) \in\left[S_{2}\left(\tau_{2}\right), 1\right]$, para todo $t_{j} \in t_{S_{j}}, j=1,2$, tal que, $\left(\widehat{S}_{1 n}\left(t_{1}\right), \widehat{S}_{2 n}\left(t_{2}\right)\right) \stackrel{\text { q.c. }}{\longrightarrow}\left(S_{1}\left(t_{1}\right), S_{2}\left(t_{2}\right)\right)$, quando $n \rightarrow \infty$. Então, para os três modelos de cópulas considerados, Clayton, Estável Positiva e Frank, quando $n \rightarrow \infty$,

$$
\dot{C}_{\alpha}\left(\widehat{S}_{1 n}, \widehat{S}_{2 n}\right) \stackrel{\text { q.c. }}{\longrightarrow} \dot{C}_{\alpha}\left(S_{1}, S_{2}\right) \text {. }
$$

\section{Prova.}

Dado que para todo $t_{j} \in t_{S_{j}}, j=1,2$, (cf. Observação 3.2.1(b))

$$
\left(\widehat{S}_{1 n}\left(t_{1}\right), \widehat{S}_{2 n}\left(t_{2}\right)\right) \stackrel{\text { q.c. }}{\longrightarrow}\left(S_{1}\left(t_{1}\right), S_{2}\left(t_{2}\right)\right), \quad \text { quando } n \rightarrow \infty \text {, }
$$

temos que para todo $\omega \in B$ com $\operatorname{Pr}\{B\}=1$,

$$
\left(\widehat{S}_{1 n}(\cdot ; \omega), \widehat{S}_{2 n}(\cdot, \omega)\right) \longrightarrow\left(S_{1}, S_{2}\right), \text { quando } n \rightarrow \infty .
$$

Pela Proposição 3.2.2, para os três modelos de cópula considerados, a função $\dot{C}_{\alpha}(\cdot, \cdot)$ é uniformemente contínua. Assim, para todo $\omega \in B$ com $\operatorname{Pr}\{B\}=1$,

$$
\dot{C}_{a}\left(\widehat{S}_{1 n}(\cdot, \omega), \widehat{S}_{2 n}(\cdot, \omega)\right) \longrightarrow \dot{C}_{\alpha}\left(S_{1}, S_{2}\right): \text { quando } n \rightarrow \infty .
$$

Portanto. quando $n \rightarrow \infty$.

$$
\dot{C}_{0}\left(\widehat{S}_{1 n}, \widehat{S}_{2 n}\right) \frac{q \cdot c \cdot}{C_{a}}\left(S_{1} \cdot S_{2}\right)
$$


No teorema seguinte estabelecemos a convergência quase certa de $\dot{C}_{\alpha_{n}^{*}}\left(\widehat{S}_{1 n}, \widehat{S}_{2 n}\right)$.

\section{Teorema 3.2.1}

Sejam $\alpha_{n}^{*}, \alpha \in \mathcal{A}$ tal que $\alpha_{n}^{*} \stackrel{\text { q.c. }}{\longrightarrow} \alpha$, quando $n \rightarrow \infty$, e sejam $\widehat{S}_{1 n}\left(t_{1}\right) \in$ $\left[S_{1}\left(\tau_{1}\right), 1\right]$ e $\widehat{S}_{2 n}\left(t_{2}\right) \in\left[S_{2}\left(\tau_{2}\right), 1\right]$, para todo $t_{j} \in t_{S_{j}}, j=1,2$, tal que $\left(\widehat{S}_{1 n}\left(t_{1}\right), \widehat{S}_{2 n}\left(t_{2}\right)\right) \stackrel{\text { q.c. }}{\longrightarrow}\left(S_{1}\left(t_{1}\right), S_{2}\left(t_{2}\right)\right)$, quando $n \rightarrow \infty$. Então, para os très modelos de cópulas considerados, Clayton, Estável Positiva e Frank, quando $n \rightarrow \infty$,

$$
\dot{C}_{\alpha_{n}^{*}}\left(\widehat{S}_{1 n}\left(t_{1}\right), \widehat{S}_{2 n}\left(t_{2}\right)\right) \stackrel{q . c .}{\longrightarrow} \dot{C}_{\alpha}\left(S_{1}\left(t_{1}\right), S_{2}\left(t_{2}\right)\right)
$$

\section{Prova.}

Usando a desigualdade triangular temos que

$$
\begin{aligned}
\left|\dot{C}_{\alpha_{n}^{*}}\left(\widehat{S}_{1 n}\left(t_{1}\right), \widehat{S}_{2 n}\left(t_{2}\right)\right)-\dot{C}_{\alpha}\left(S_{1}\left(t_{1}\right), S_{2}\left(t_{2}\right)\right)\right| \leq \\
\leq\left|\dot{C}_{\alpha_{n}^{*}}\left(\widehat{S}_{1 n}\left(t_{1}\right), \widehat{S}_{2 n}\left(t_{2}\right)\right)-\dot{C}_{\alpha}\left(\widehat{S}_{1 n}\left(t_{1}\right), \widehat{S}_{2 n}\left(t_{2}\right)\right)\right| \\
+\left|\dot{C}_{\alpha}\left(\widehat{S}_{1 n}\left(t_{1}\right), \widehat{S}_{2 n}\left(t_{2}\right)\right)-\dot{C}_{\alpha}\left(S_{1}\left(t_{1}\right), S_{2}\left(t_{2}\right)\right)\right|
\end{aligned}
$$

Para o primeiro termo à direita em (3.2.11), de (3.2.9) no Lema 3.2.1 temos que para todo $t_{j} \in t_{S_{j}}, j=1,2$, para todo $\varepsilon>0$ e para $n \geq n_{0}(\varepsilon)$,

$$
\left|\dot{C}_{\alpha_{n}^{*}}\left(\widehat{S}_{1 n}\left(t_{1}\right), \widehat{S}_{2 n}\left(t_{2}\right)\right)-\dot{C}_{\alpha}\left(\widehat{S}_{1 n}\left(t_{1}\right), \widehat{S}_{2 n}\left(t_{2}\right)\right)\right|<\varepsilon \text {. (q.c.) }
$$

No segundo termo à direita em (3.2.11), do Lema 3.2.2, temos que para todo $t_{j} \in t_{S_{j}}, j=1.2$. para todo $\Xi>0$ e para $n \geq n_{0}(\Xi)$.

$$
\left|\dot{C}_{n}\left(\widehat{S}_{1 n}\left(t_{1}\right) \cdot \widehat{S}_{2 n}\left(t_{2}\right)\right)-\dot{C}_{n}\left(S_{1}\left(t_{1}\right) \cdot S_{2}\left(t_{2}\right)\right)\right|<\equiv . \quad(q . c .)
$$


Logo, de (3.2.12) e (3.2.13) segue que para todo $t_{j} \in t_{S_{j}}, j=1,2$, para todo $\varepsilon>0$ e para $n \geq n_{0}(\varepsilon)$

$$
\left|\dot{C}_{\alpha_{n}^{*}}\left(\widehat{S}_{1 n}\left(t_{1}\right), \widehat{S}_{2 n}\left(t_{2}\right)\right)-\dot{C}_{\alpha}\left(S_{1}\left(t_{1}\right), S_{2}\left(t_{2}\right)\right)\right|<2 \varepsilon \text {. (q.c.) }
$$

Assim, obtemos (3.2.10), i.e., $\dot{C}_{\alpha_{n}^{*}}\left(\widehat{S}_{1 n}\left(t_{1}\right), \widehat{S}_{2 n}\left(t_{2}\right)\right)$ é um estimador fortemente consistente de $\dot{C}_{\alpha}\left(S_{1}\left(t_{1}\right), S_{2}\left(t_{2}\right)\right)$, para todo $t_{j} \in t_{S_{j}}, j=1,2$.

\section{Observação 3.2 .2}

Notamos que para os três modelos de cópulas considerados $\dot{C}_{\alpha}\left(S_{1}\left(t_{1}\right), S_{2}\left(t_{2}\right)\right)$ é limitada, pois a função $\dot{C}_{\alpha}(\cdot, \cdot)$ é contínua e é definida no compacto $\left[S_{1}\left(\tau_{1}\right), 1\right] \times\left[S_{2}\left(\tau_{2}\right), 1\right]$ (veja Corolário B.1). Além disso, $\alpha$ é assumido a estar restrito a un subconjunto compacto de $\mathcal{A}$.

\subsubsection{Consistência forte de $C_{o}\left(\widehat{S}_{1 n}, \widehat{S}_{2 n}\right)$}

Nesta seção estudamos a consistência forte do funcional $C_{o}\left(\widehat{S}_{1 n}, \widehat{S}_{2 n} ; t\right)$ definido em (3.2.5). Para estabelecer esta convergência mostramos inicialmente que o funcional $C_{o}$ é contínuo.

\section{Teorema 3.2 .2}

Seja $\mathcal{S} \subset D[0, \infty]$ o espaço de todas as funções de sobrevivência caglad sobre $[0, x]$, munido com a norma do supremo. Então o funcional $C_{o}: D[0, \infty] \times$ $D[0, x] \mapsto[0.1]$, definiclo por

$$
C_{0}\left(H_{1}(\cdot) \cdot H_{2}(\cdot): t\right)=C_{0}\left(H_{1}\left(t_{1}\right) \cdot H_{2}\left(t_{2}\right)\right)
$$


em que $H_{1}, H_{2} \in \mathcal{S}$, é um funcional contínuo.

\section{Prova.}

Considere uma sequência $\left(H_{1 n}, H_{2 n}\right)$ em $\mathcal{S} \times \mathcal{S} \subset D[0, \infty] \times D[0, \infty]$, tal que $\left\|H_{j n}-H_{j}\right\| \rightarrow 0$, quando $n \rightarrow \infty, j=1,2$, para algum $\left(H_{1}, H_{2}\right) \mathrm{em}$ $\mathcal{S} \times \mathcal{S} \subset D[0, \infty] \times D[0, \infty]$. Usando a desigualdade triangular, temos que

$$
\begin{aligned}
\left\|C_{o}\left(H_{1 n}, H_{2 n}\right)-C_{o}\left(H_{1}, H_{2}\right)\right\| & \leq \\
\leq & \left\|C_{o}\left(H_{1 n}, H_{2 n}\right)-C_{o}\left(H_{1}, H_{2 n}\right)\right\|+\left\|C_{o}\left(H_{1}, H_{2 n}\right)-C_{o}\left(H_{1}, H_{2}\right)\right\| .
\end{aligned}
$$

Suponha que $H_{1}\left(t_{1}\right) \leq H_{1 n}\left(t_{1}\right)$ para todo $t_{1} \in t_{S_{1}}$. Então, como $C_{\alpha}(\cdot, \cdot)$ é uma função não-crescente em seus argumentos (função de sobrevivência) temos que para qualquer $H_{2 n}\left(t_{2}\right) \in \mathcal{S}$,

$$
\begin{aligned}
0 \leq C_{\alpha}\left(H_{1 n}\left(t_{1}\right), H_{2 n}\left(t_{2}\right)\right) & -C_{\alpha}\left(H_{1}\left(t_{1}\right), H_{2 n}\left(t_{2}\right)\right) \\
\leq & C_{\alpha}\left(H_{1 n}\left(t_{1}\right), 1\right)-C_{\alpha}\left(H_{1}\left(t_{1}\right), 1\right)=H_{1 n}\left(t_{1}\right)-H_{1}\left(t_{1}\right) .
\end{aligned}
$$

No caso de $H_{1 n}\left(t_{1}\right) \leq H_{1}\left(t_{1}\right)$ para todo $t_{1} \in t_{S_{1}}$,

$$
\begin{aligned}
0 \leq C_{\alpha}\left(H_{1}\left(t_{1}\right), H_{2 n}\left(t_{2}\right)\right) & -C_{\alpha}\left(H_{1 n}\left(t_{1}\right), H_{2 n}\left(t_{2}\right)\right) \\
\leq & C_{\alpha}\left(H_{1}\left(t_{1}\right), 1\right)-C_{\alpha}\left(H_{1 n}\left(t_{1}\right), 1\right)=H_{1}\left(t_{1}\right)-H_{1 n}\left(t_{1}\right) .
\end{aligned}
$$

Logo,

$$
\left|C_{\alpha}\left(H_{1 n}\left(t_{1}\right), H_{2 n}\left(t_{2}\right)\right)-C_{\alpha}\left(H_{1}\left(t_{1}\right), H_{2 n}\left(t_{2}\right)\right)\right| \leq\left|H_{1 n}\left(t_{1}\right)-H_{1}\left(t_{1}\right)\right|:
$$

e.

$$
\begin{aligned}
\sup _{t_{1} \in t_{S_{1}}} \sup _{t_{2} \in t_{S_{2}}} \mid C_{\alpha}\left(H_{1 n}\left(t_{1}\right) \cdot H_{2 n}\left(t_{2}\right)\right) & -C_{\alpha}\left(H_{1}\left(t_{1}\right) \cdot H_{2 n}\left(t_{2}\right)\right) \mid \\
\leq & \sup _{n_{1} \in t_{s_{1}}}\left|H_{1 n}\left(t_{1}\right)-H_{1}\left(t_{1}\right)\right| .
\end{aligned}
$$


Analogamente, para qualquer $H_{1}\left(t_{1}\right) \in \mathcal{S}$;

$$
\begin{aligned}
\sup _{t_{2} \in t_{S_{2}}} \sup _{t_{1} \in t_{S_{1}}} \mid C_{\alpha}\left(H_{1}\left(t_{1}\right), H_{2 n}\left(t_{2}\right)\right) & -C_{\alpha}\left(H_{1}\left(t_{1}\right), H_{2}\left(t_{2}\right)\right) \mid \\
\leq & \sup _{t_{2} \in t_{S_{2}}}\left|H_{2 n}\left(t_{2}\right)-H_{2}\left(t_{2}\right)\right| .
\end{aligned}
$$

Assim,

$$
\begin{aligned}
\left\|C_{o}\left(H_{1 n}, H_{2 n}\right)-C_{o}\left(H_{1}, H_{2}\right)\right\| \\
\quad \leq \sup _{t_{1} \in t_{S_{1}}}\left|H_{1 n}\left(t_{1}\right)-H_{1}\left(t_{1}\right)\right|+\sup _{t_{2} \in t_{S_{2}}}\left|H_{2 n}\left(t_{2}\right)-H_{2}\left(t_{2}\right)\right| \\
\quad=\left\|H_{1 n}-H_{1}\right\|+\left\|H_{2 n}-H_{2}\right\| \rightarrow 0 \text {, quando } n \rightarrow \infty .
\end{aligned}
$$

Assim, o funcional $C_{o}$ é contínuo em $\mathcal{S} \times \mathcal{S}$.

\section{Corolário 3.2.1}

$C_{o}\left(\widehat{S}_{1 n}, \widehat{S}_{2 n}\right)$ é um estimador fortemente consistente de $C_{o}\left(S_{1}, S_{2}\right)$.

\section{Prova.}

É uma consequência direta do teorema anterior e da consistência forte do estimador Bayesiano do vetor de sobrevivências marginais $\left(\widehat{S}_{1 n}, \widehat{S}_{2 n}\right)$ (cf. Observação 3.2.1(b)).

\subsubsection{Convergência fraca de $C_{o}\left(\widehat{S}_{1 n}, \widehat{S}_{2 n}\right)$}

Considere a seguinte expansão de von Mises (von Mises, 1947) para o funcional $C_{o}$, avaliado em $\left(\widehat{S}_{1 n}, \widehat{S}_{2 n}\right)$. em torno de $\left(S_{1}, S_{2}\right)$. Denotando 
por $C_{o\left(S_{1}, S_{2}\right)}^{\prime}$ a derivada do funcional em $\left(S_{1}(\cdot), S_{2}(\cdot)\right)$ podemos escrever $C_{o}\left(\widehat{S}_{1 n}(\cdot), \widehat{S}_{2 n}(\cdot) ; t\right)$ por

$$
\begin{aligned}
C_{o}\left(\widehat{S}_{1 n}(\cdot), \widehat{S}_{2 n}(\cdot) ; t\right)= & C_{o}\left(S_{1}(\cdot), S_{2}(\cdot) ; t\right) \\
& +C_{o\left(S_{1}, S_{2}\right)}^{\prime}\left(\widehat{S}_{1 n}(\cdot)-S_{1}(\cdot), \widehat{S}_{2 n}(\cdot)-S_{2}(\cdot) ; t\right) \\
& +\operatorname{Res}\left(\widehat{S}_{1 n}(\cdot)-S_{1}(\cdot), \widehat{S}_{2 n}(\cdot)-S_{2}(\cdot): C_{o}: t\right),
\end{aligned}
$$

em que $\operatorname{Res}\left(\cdot, \cdot ; C_{o}, t\right)$ corresponde ao resto da aproximação da expansão em primeira ordem, $t=\left(t_{1}, t_{2}\right)$ e $t_{j} \in t_{S_{j}}$, para $t_{S_{j}}=\left[0, \tau_{j}\right]$ com $\tau_{j}=\sup \left\{t_{j}: S_{j}\left(t_{j}\right)>0\right\}, j=1,2$.

O teorema seguinte estabelece que o funcional $C_{o}$ é Hadamard diferenciável. Além disso, mostramos que o resto da expansão é um termo desprezível.

\section{Teorema 3.2.3}

Seja a função de sobrevivência bivariada $S\left(t_{1}, t_{2}\right)=C_{\alpha}\left(S_{1}\left(t_{1}\right): S_{2}\left(t_{2}\right)\right)$ com $S\left(t_{1}, 0\right)=S_{1}\left(t_{1}\right)$ e $S\left(0, t_{2}\right)=S_{2}\left(t_{2}\right)$, funções de sobrevivência marginais, para todo $t_{j} \in t_{S_{j}}, j=1,2$. Seja o funcional $C_{o}: D[0, \infty] \times D[0, \infty] \mapsto[0,1]$ dado em (3.2.5) com $H_{j} \in \mathcal{S} \subset D[0, \infty], j=1,2$. Então;

(a) o funcional $C_{o}$ é Hadamard diferenciável em $\left(S_{1}(\cdot), S_{2}(\cdot)\right)$. A derivada é dada por

$$
\begin{aligned}
& C_{o\left(S_{1}, S_{2}\right)}^{\prime}\left(H_{1}(\cdot) \cdot H_{2}(\cdot): t\right)=c_{\alpha}\left(S_{1}\left(t_{1}\right), S_{2}\left(t_{2}\right)\right) \cdot H_{1}\left(t_{1}\right) \cdot H_{2}\left(t_{2}\right) \\
& +\frac{\partial C_{\alpha}\left(S_{1}\left(t_{1}\right) \cdot S_{2}\left(t_{2}\right)\right)}{\partial x} \cdot H_{1}\left(t_{1}\right)+\frac{\partial C_{\alpha}\left(S_{1}\left(t_{1}\right) \cdot S_{2}\left(t_{2}\right)\right)}{\partial y} \cdot H_{2}\left(t_{2}\right)
\end{aligned}
$$

em que $c_{a}$ denota a correspondente função de densidade da cópula $C_{\alpha}$. 
(b) $n^{1 / 2} \operatorname{Res}\left(\widehat{S}_{1 n}(\cdot)-S_{1}(\cdot), \widehat{S}_{2 n}(\cdot)-S_{2}(\cdot) ; C_{o}, t\right) \stackrel{P}{\longrightarrow} 0$, quando $n \rightarrow \infty$ e portanto

$$
\begin{aligned}
n^{1 / 2}\left[C_{o}\left(\widehat{S}_{1 n}(\cdot), \widehat{S}_{2 n}(\cdot) ; t\right)-C_{o}\left(S_{1}(\cdot), S_{2}(\cdot): t\right)\right]= \\
=n^{1 / 2} C_{o\left(S_{1}, S_{2}\right)}^{\prime}\left(\widehat{S}_{1 n}(\cdot)-S_{1}(\cdot), \widehat{S}_{2 n}(\cdot)-S_{2}(\cdot) ; t\right)+o_{p}(1) .
\end{aligned}
$$

quando $n \rightarrow \infty$.

\section{Prova.}

(a) Seguindo a van der Vaart e Wellner (1996), página 389, podemos decompor $S\left(t_{1}, t_{2}\right)$ nas seguintes duas aplicações para todo $t_{j} \in t_{S_{j}}$, $j=1,2$,

(i) $C_{\alpha} \mapsto\left(C_{\alpha}, u, v\right)=\left(C_{\alpha}, S_{1}\left(t_{1}\right), S_{2}\left(t_{2}\right)\right)$

(ii) $\left(C_{\alpha}, S_{1}\left(t_{1}\right), S_{2}\left(t_{2}\right)\right) \mapsto C_{\alpha} \circ\left(S_{1}\left(t_{1}\right), S_{2}\left(t_{2}\right)\right)=C_{o}\left(S_{1}(\cdot), S_{2}(\cdot) ; \boldsymbol{t}\right)$

A primeira aplicação, $C_{\alpha} \mapsto\left(C_{\alpha}, u, v\right)=\left(C_{\alpha}, S_{1}\left(t_{1}\right), S_{2}\left(t_{2}\right)\right)$ é linear e contínua, e portanto Hadamard diferenciável (a prova é adiada para o final deste capítulo).

A segunda aplicação, $\left(C_{\alpha}, S_{1}\left(t_{1}\right), S_{2}\left(t_{2}\right)\right) \mapsto C_{\alpha} \circ\left(S_{1}\left(t_{1}\right), S_{2}\left(t_{2}\right)\right)=$ $C_{o}\left(S_{1}(\cdot), S_{2}(\cdot) ; t\right)$, é Hadamard diferenciável pelo Lema 3.9.27 em van der Vaart e Wellner (1996), considerando $\mathcal{X}=t_{S_{1}} \times t_{S_{2}}, \mathcal{Y}=\left[S_{1}\left(\tau_{1}\right), 1\right] \times$ $\left[S_{2}\left(\tau_{2}\right), 1\right] \subset \mathbb{R}^{2}$ e $\mathcal{Z}=\mathbb{R}$. Logo, aplicando a regra da cadeia, segue o resultado.

(b) Considere a partir de (3.2.14);

$$
\begin{aligned}
\operatorname{Res}\left(\widehat{S}_{1 n}(\cdot)-S_{1}(\cdot) \cdot \widehat{S}_{2 n}(\cdot)-S_{2}(\cdot): C_{0} \cdot t\right)= \\
=C_{0}\left(\widehat{S}_{1 n}(\cdot) \cdot \widehat{S}_{2 n}(\cdot): t\right)-C_{o}\left(S_{1}(\cdot), S_{2}(\cdot): t\right) \\
\quad-C_{0\left(S_{1} \ldots S_{2}\right)}^{\prime}\left(\widehat{S}_{1 n}(\cdot)-S_{1}(\cdot) \cdot \widehat{S}_{2 n}(\cdot)-S_{2}(\cdot): t\right)
\end{aligned}
$$




$$
\begin{aligned}
= & C_{\alpha}\left(\widehat{S}_{1 n}\left(t_{1}\right), \widehat{S}_{2 n}\left(t_{2}\right)\right)-C_{\alpha}\left(S_{1}\left(t_{1}\right), S_{2}\left(t_{2}\right)\right) \\
& -c_{\alpha}\left(S_{1}\left(t_{1}\right), S_{2}\left(t_{2}\right)\right) \cdot\left(\widehat{S}_{1 n}\left(t_{1}\right)-S_{1}\left(t_{1}\right)\right) \cdot\left(\widehat{S}_{2 n}\left(t_{2}\right)-S_{2}\left(t_{2}\right)\right) \\
& -\frac{\partial C_{\alpha}\left(S_{1}\left(t_{1}\right), S_{2}\left(t_{2}\right)\right)}{\partial x} \cdot\left(\widehat{S}_{1 n}\left(t_{1}\right)-S_{1}\left(t_{1}\right)\right) \\
& -\frac{\partial C_{\alpha}\left(S_{1}\left(t_{1}\right), S_{2}\left(t_{2}\right)\right)}{\partial y} \cdot\left(\widehat{S}_{2 n}\left(t_{2}\right)-S_{2}\left(t_{2}\right)\right) .
\end{aligned}
$$

Agora, decomponha $C_{\alpha}\left(\widehat{S}_{1 n}\left(t_{1}\right), \widehat{S}_{2 n}\left(t_{2}\right)\right)-C_{\alpha}\left(S_{1}\left(t_{1}\right), S_{2}\left(t_{2}\right)\right)$ em três termos, $R_{1 n}, R_{2 n}$ e $R_{3 n}$, isto é,

$$
\begin{aligned}
C_{\alpha}\left(\widehat{S}_{1 n}\left(t_{1}\right), \widehat{S}_{2 n}\left(t_{2}\right)\right)-C_{\alpha}\left(S_{1}\left(t_{1}\right), S_{2}\left(t_{2}\right)\right)= & +C_{\alpha}\left(S_{1}\left(t_{1}\right), S_{2}\left(t_{2}\right)\right) \\
= & C_{\alpha}\left(\widehat{S}_{1 n}\left(t_{1}\right), \widehat{S}_{2 n}\left(t_{2}\right)\right)-C_{\alpha}\left(S_{1}\left(t_{1}\right), \widehat{S}_{2 n}\left(t_{2}\right)\right)-C_{\alpha}\left(\widehat{S}_{1 n}\left(t_{1}\right), S_{2}\left(t_{2}\right)\right) \\
& +C_{\alpha}\left(\widehat{S}_{1 n}\left(t_{1}\right), S_{2}\left(t_{2}\right)\right)-C_{\alpha}\left(S_{1}\left(t_{1}\right), S_{2}\left(t_{2}\right)\right) \\
& +C_{\alpha}\left(S_{1}\left(t_{1}\right), \widehat{S}_{2 n}\left(t_{2}\right)\right)-C_{\alpha}\left(S_{1}\left(t_{1}\right), S_{2}\left(t_{2}\right)\right) \\
= & R_{1 n}\left(\widehat{S}_{1 n}\left(t_{1}\right), \widehat{S}_{2 n}\left(t_{2}\right), S_{1}\left(t_{1}\right), S_{2}\left(t_{2}\right)\right) \\
& +R_{2 n}\left(\widehat{S}_{1 n}\left(t_{1}\right), S_{1}\left(t_{1}\right), S_{2}\left(t_{2}\right)\right) \\
& +R_{3 n}\left(\widehat{S}_{2 n}\left(t_{2}\right), S_{1}\left(t_{1}\right), S_{2}\left(t_{2}\right)\right) .
\end{aligned}
$$

Substituindo (3.2.16) em (3.2.15),

$$
\begin{aligned}
& \operatorname{Res}\left(\widehat{S}_{1 n}(\cdot)-S_{1}(\cdot), \widehat{S}_{2 n}(\cdot)-S_{2}(\cdot) ; C_{o}: t\right)= \\
& =R_{1 n}\left(\widehat{S}_{1 n}\left(t_{1}\right), \widehat{S}_{2 n}\left(t_{2}\right), S_{1}\left(t_{1}\right), S_{2}\left(t_{2}\right)\right) \\
& \quad-c_{\alpha}\left(S_{1}\left(t_{1}\right), S_{2}\left(t_{2}\right)\right) \cdot\left(\widehat{S}_{1 n}\left(t_{1}\right)-S_{1}\left(t_{1}\right)\right) \cdot\left(\widehat{S}_{2 n}\left(t_{2}\right)-S_{2}\left(t_{2}\right)\right) \\
& \quad+R_{2 n}\left(\widehat{S}_{1 n}\left(t_{1}\right), S_{1}\left(t_{1}\right), S_{2}\left(t_{2}\right)\right) \\
& \quad-\frac{\partial C_{0}\left(S_{1}\left(t_{1}\right) \cdot S_{2}\left(t_{2}\right)\right)}{\partial x} \cdot\left(\widehat{S}_{1 n}\left(t_{1}\right)-S_{1}\left(t_{1}\right)\right) \\
& +R_{3 n}\left(\widehat{S}_{2 n}\left(t_{2}\right), S_{1}\left(t_{1}\right) \cdot S_{2}\left(t_{2}\right)\right) \\
& \quad-\frac{\partial C_{n}\left(S_{1}\left(t_{1}\right) \cdot S_{2}\left(t_{2}\right)\right)}{\partial y} \cdot\left(\widehat{S}_{2 n}\left(t_{2}\right)-S_{2}\left(t_{2}\right)\right) \cdot(3.2 .17)
\end{aligned}
$$


Como $\widehat{S}_{1 n}\left(t_{1}\right) \stackrel{q . c .}{\longrightarrow} S_{1}\left(t_{1}\right)$, e $\widehat{S}_{2 n}\left(t_{\cdot 2}\right) \stackrel{q . c .}{\longrightarrow} S_{2}\left(t_{2}\right)$ temos que

$$
\begin{gathered}
R_{1 n}\left(\widehat{S}_{1 n}\left(t_{1}\right), \widehat{S}_{2 n}\left(t_{2}\right), S_{1}\left(t_{1}\right), S_{2}\left(t_{2}\right)\right)=C_{\alpha}\left(\widehat{S}_{1 n}\left(t_{1}\right), \widehat{S}_{2 n}\left(t_{2}\right)\right) \\
\quad-C_{\alpha}\left(S_{1}\left(t_{1}\right), \widehat{S}_{2 n}\left(t_{2}\right)\right)-C_{\alpha}\left(\widehat{S}_{1 n}\left(t_{1}\right), S_{2}\left(t_{2}\right)\right)+C_{\alpha}\left(S_{1}\left(t_{1}\right), S_{2}\left(t_{2}\right)\right) \\
\approx c_{\alpha}\left(S_{1}\left(t_{1}\right), S_{2}\left(t_{2}\right)\right) \cdot\left(\widehat{S}_{1 n}\left(t_{1}\right)-S_{1}\left(t_{1}\right)\right) \cdot\left(\widehat{S}_{2 n}\left(t_{2}\right)-S_{2}\left(t_{2}\right)\right)
\end{gathered}
$$

assim, para todo $\varepsilon>0$ e para $n \geq n_{0}(\varepsilon)$ podemos escrever;

$$
\left|\frac{R_{1 n}\left(\widehat{S}_{1 n}\left(t_{1}\right), \widehat{S}_{2 n}\left(t_{2}\right), S_{1}\left(t_{1}\right), S_{2}\left(t_{2}\right)\right)}{\left(\widehat{S}_{1 n}\left(t_{1}\right)-S_{1}\left(t_{1}\right)\right) \cdot\left(\widehat{S}_{2 n}\left(t_{2}\right)-S_{2}\left(t_{2}\right)\right)}-c_{\alpha}\left(S_{1}\left(t_{1}\right), S_{2}\left(t_{2}\right)\right)\right| \leq \varepsilon .
$$

Logo, da expressão anterior, considerando $n^{1 / 2}\left(\widehat{S}_{1 n}\left(t_{1}\right)-S_{1}\left(t_{1}\right)\right)=$ $O_{p}(1)$ e $\left(\widehat{S}_{2 n}\left(t_{2}\right)-S_{2}\left(t_{2}\right)\right)=o_{p}(1)$ (cf. Observação 3.2.1(b)), temos que

$$
\begin{aligned}
n^{1 / 2} \mid R_{1 n}\left(\widehat{S}_{1 n}\left(t_{1}\right), \widehat{S}_{2 n}\left(t_{2}\right)\right. & \left., S_{1}\left(t_{1}\right), S_{2}\left(t_{2}\right)\right)- \\
c_{\alpha}\left(S_{1}\left(t_{1}\right)\right. & \left., S_{2}\left(t_{2}\right)\right) \cdot\left(\widehat{S}_{1 n}\left(t_{1}\right)-S_{1}\left(t_{1}\right)\right) \cdot\left(\widehat{S}_{2 n}\left(t_{2}\right)-S_{2}\left(t_{2}\right)\right) \mid \\
& \leq n^{1 / 2}\left|\widehat{S}_{1 n}\left(t_{1}\right)-S_{1}\left(t_{1}\right)\right| \cdot\left|\widehat{S}_{2 n}\left(t_{2}\right)-S_{2}\left(t_{2}\right)\right| \cdot \varepsilon \\
& =o_{p}(1)
\end{aligned}
$$

Do segundo termo em (3.2.17) temos que para $\widehat{S}_{1 n}\left(t_{1}\right) \stackrel{\text { q.c. }}{\longrightarrow} S_{1}\left(t_{1}\right)$,

$$
\begin{aligned}
R_{2 n}\left(\widehat{S}_{1 n}\left(t_{1}\right), S_{1}\left(t_{1}\right), S_{2}\left(t_{2}\right)\right) & =C_{\alpha}\left(\widehat{S}_{1 n}\left(t_{1}\right), S_{2}\left(t_{2}\right)\right)-C_{\alpha}\left(S_{1}\left(t_{1}\right), S_{2}\left(t_{2}\right)\right) \\
& \approx \frac{\partial C_{\alpha}\left(S_{1}\left(t_{1}\right), S_{2}\left(t_{2}\right)\right)}{\partial x} \cdot\left(\widehat{S}_{1 n}\left(t_{1}\right)-S_{1}\left(t_{1}\right)\right) .
\end{aligned}
$$

assim, para todo $\varepsilon>0$ e para $n \geq n_{0}(\Xi)$ podemos escrever;

$$
\left|\frac{R_{2 n}\left(\widehat{S}_{1 n}\left(t_{1}\right) \cdot S_{1}\left(t_{1}\right) \cdot S_{2}\left(t_{2}\right)\right)}{\widehat{S}_{1 n}\left(t_{1}\right)-S_{1}\left(t_{1}\right)}-\frac{\partial C_{n}\left(S_{1}\left(t_{1}\right) \cdot S_{2}\left(t_{2}\right)\right)}{\partial x}\right| \leq \xi .
$$


Logo, da expressão anterior, considerando $n^{1 / 2}\left(\widehat{S}_{1 n}\left(t_{1}\right)-S_{1}\left(t_{1}\right)\right)=$ $O_{p}(1)$ (cf. Observaşão 3.2.1(b)), temos que

$$
\begin{aligned}
n^{1 / 2} \mid R_{2 n}\left(\widehat{S}_{1 n}\left(t_{1}\right), S_{1}\left(t_{1}\right), S_{2}\left(t_{2}\right)\right) & -\frac{\partial C_{\alpha}\left(S_{1}\left(t_{1}\right), S_{2}\left(t_{2}\right)\right)}{\partial x} \cdot\left(\widehat{S}_{1 n}\left(t_{1}\right)-S_{1}\left(t_{1}\right)\right) \mid \\
& \leq n^{1 / 2}\left|\widehat{S}_{1 n}\left(t_{1}\right)-S_{1}\left(t_{1}\right)\right| \cdot \varepsilon \\
& =o_{p}(1)
\end{aligned}
$$

Analogamente, para o terceiro termo em (3.2.17) temos que para $\widehat{S}_{2 n}\left(t_{2}\right) \stackrel{q . c .}{\longrightarrow} S_{2}\left(t_{2}\right)$,

$$
\begin{aligned}
R_{3 n}\left(\widehat{S}_{2 n}\left(t_{2}\right), S_{1}\left(t_{1}\right), S_{2}\left(t_{2}\right)\right) & =C_{\alpha}\left(S_{1}\left(t_{1}\right), \widehat{S}_{2 n}\left(t_{2}\right)\right)-C_{\alpha}\left(S_{1}\left(t_{1}\right), S_{2}\left(t_{2}\right)\right) \\
& \approx \frac{\partial C_{\alpha}\left(S_{1}\left(t_{1}\right), S_{2}\left(t_{2}\right)\right)}{\partial y} \cdot\left(\widehat{S}_{2 n}\left(t_{2}\right)-S_{2}\left(t_{2}\right)\right),
\end{aligned}
$$

assim, para todo $\varepsilon>0$ e para $n \geq n_{0}(\varepsilon)$ podemos escrever,

$$
\left|\frac{R_{3 n}\left(\widehat{S}_{2 n}\left(t_{2}\right), S_{1}\left(t_{1}\right), S_{2}\left(t_{2}\right)\right)}{\widehat{S}_{2 n}\left(t_{2}\right)-S_{2}\left(t_{2}\right)}-\frac{\partial C_{\alpha}\left(S_{1}\left(t_{1}\right), S_{2}\left(t_{2}\right)\right)}{\partial y}\right| \leq \varepsilon .
$$

Logo, da expressão anterior, considerando $n^{1 / 2}\left(\widehat{S}_{2 n}\left(t_{2}\right)-S_{2}\left(t_{2}\right)\right)=$ $O_{p}(1)$ (cf. Observação 3.2.1(b)), temos que

$$
\begin{aligned}
n^{1 / 2} \mid R_{3 n}\left(\widehat{S}_{2 n}\left(t_{2}\right), S_{1}\left(t_{1}\right), S_{2}\left(t_{2}\right)\right) & -\frac{\partial C_{\alpha}\left(S_{1}\left(t_{1}\right), S_{2}\left(t_{2}\right)\right)}{\partial y} \cdot\left(\widehat{S}_{2 n}\left(t_{2}\right)-S_{2}\left(t_{2}\right)\right) \mid \\
& \leq n^{1 / 2}\left|\widehat{S}_{2 n}\left(t_{2}\right)-S_{2}\left(t_{2}\right)\right| \cdot \Xi \\
& =o_{p}(1)
\end{aligned}
$$

Portanto: substituindo (3.2.18). (3.2.19) e (3.2.20) em (3.2.17) obtemos $n^{1 / 2} \operatorname{Res}\left(\widehat{S}_{1 n}(\cdot)-S_{1}(\cdot) . \widehat{S}_{2 n}(\cdot)-S_{2}(\cdot): C_{o}, t\right) \stackrel{P}{\longrightarrow} 0$. quando $n \rightarrow \infty$. 


\section{Observação 3.2 .3}

(a) Notamos que a derivada de Hadamard obtida acima difere da derivada de Hadamard dada em van der Vaart e Wellner (1996), Lema 3.9.28. Embora a decomposição da cópula, em aplicações Hadamard diferenciáveis, seja similar, os enfoques são distintos. Van der Vaart e Wellner consideram a cópula como uma função de uma função de distribuição bivariada $H$, enquanto que nesta seção a cópula é analisada como um funcional de $\left(S_{1}, S_{2}\right)$. Além disso, em van der Vaart e Wellner a derivada é em relação a $\left(t_{1}, t_{2}\right)$ enquanto que acima a derivada é em relação a $\left(S_{1}, S_{2}\right)$.

(b) Destacamos que no funcional $C_{o}$ estamos considerando o parâmetro de dependência $\alpha$ fixado e definido em um subconjunto compacto de $\mathcal{A}$. No caso geral de $\alpha \in \mathcal{A}$, os resultados desta seção podem ser obtidos considerando o funcional como um funcional extendido, veja Ren e Sen (1991).

Baseado no Teorema 3.2.3 mostraremos a seguir que o estimador $C_{\alpha}\left(\widehat{S}_{1 n}\left(t_{1}\right), \widehat{S}_{2 n}\left(t_{2}\right)\right)$ de $C_{\alpha}\left(S_{1}\left(t_{1}\right), S_{2}\left(t_{2}\right)\right)$ é assintoticamente normal.

\section{Teorema 3.2 .4}

Seja $C_{\alpha}\left(\widehat{S}_{1 n}\left(t_{1}\right), \widehat{S}_{2 n}\left(t_{2}\right)\right)=C_{o}\left(\widehat{S}_{1 n}(\cdot), \widehat{S}_{2 n}(\cdot) ; t\right)$ um estimador de $C_{\alpha}\left(S_{1}\left(t_{1}\right) \cdot S_{2}\left(t_{2}\right)\right)=C_{o}\left(S_{1}(\cdot) \cdot S_{2}(\cdot): t\right)$. Se o funcional $C_{o}: D[0, x] \times$ $D[0 . x] \mapsto[0.1]$ : é Hadamard diferenciável em $\left(S_{1} . S_{2}\right)$, então

$$
\left.n^{12} C_{a}\left(\widehat{S}_{1 n}\left(t_{1}\right) . \widehat{S}_{2 n}\left(t_{2}\right)\right)-C_{n}\left(S_{1}\left(t_{1}\right), S_{2}\left(t_{2}\right)\right)\right] \stackrel{D}{-} N\left(0 . \sigma_{S}^{2}\right)
$$


quando $n \rightarrow \infty$, com,

$$
\begin{aligned}
\sigma_{S}^{2}= & \left(\frac{\partial C_{\alpha}\left(S_{1}\left(t_{1}\right), S_{2}\left(t_{2}\right)\right)}{\partial x}\right)^{2} \cdot \sigma_{S_{1}}^{2}+\left(\frac{\partial C_{\alpha}\left(S_{1}\left(t_{1}\right), S_{2}\left(t_{2}\right)\right)}{\partial y}\right)^{2} \cdot \sigma_{S_{2}}^{2} \\
& +2\left(\frac{\partial^{2} C_{\alpha}\left(S_{1}\left(t_{1}\right), S_{2}\left(t_{2}\right)\right)}{\partial x \partial y}\right) \cdot \operatorname{Cov}\left[\widehat{S}_{1 n}\left(t_{1}\right), \widehat{S}_{2 n}\left(t_{2}\right)\right]
\end{aligned}
$$

\section{Prova.}

Pelo Teorema 3.2.3, temos para $n \rightarrow \infty$,

$$
\begin{aligned}
& n^{1 / 2}\left[C_{\alpha}\left(\widehat{S}_{1 n}\left(t_{1}\right), \widehat{S}_{2 n}\left(t_{2}\right)\right)-C_{\alpha}\left(S_{1}\left(t_{1}\right), S_{2}\left(t_{2}\right)\right)\right]= \\
& \quad=n^{1 / 2} C_{\alpha\left(S_{1}, S_{2}\right)}^{\prime}\left(\widehat{S}_{1 n}\left(t_{1}\right)-S_{1}\left(t_{1}\right), \widehat{S}_{2 n}\left(t_{2}\right)-S_{2}\left(t_{2}\right)\right)+o_{p}(1) .
\end{aligned}
$$

Como

$$
n^{1 / 2} c_{\alpha}\left(S_{1}\left(t_{1}\right), S_{2}\left(t_{2}\right)\right) \cdot\left(\widehat{S}_{1 n}\left(t_{1}\right)-S_{1}\left(t_{1}\right)\right) \cdot\left(\widehat{S}_{2 n}\left(t_{2}\right)-S_{2}\left(t_{2}\right)\right)=o_{p}(1),
$$

temos que,

$$
\begin{aligned}
n^{1 / 2}\left[C _ { \alpha } \left(\widehat{S}_{1 n}\left(t_{1}\right),\right.\right. & \left.\left.\widehat{S}_{2 n}\left(t_{2}\right)\right)-C_{\alpha}\left(S_{1}\left(t_{1}\right), S_{2}\left(t_{2}\right)\right)\right]= \\
= & n^{1 / 2}\left(\frac{\partial C_{\alpha}\left(S_{1}\left(t_{1}\right), S_{2}\left(t_{2}\right)\right)}{\partial x}\right) \cdot\left[\widehat{S}_{1 n}\left(t_{1}\right)-S_{1}\left(t_{1}\right)\right] \\
& \quad+n^{1 / 2}\left(\frac{\partial C_{\alpha}\left(S_{1}\left(t_{1}\right), S_{2}\left(t_{2}\right)\right)}{\partial y}\right) \cdot\left[\widehat{S}_{2 n}\left(t_{2}\right)-S_{2}\left(t_{2}\right)\right]+o_{p}(1) .
\end{aligned}
$$

Considerando que quando $n \rightarrow \infty$, (cf. Observação 3.2.1(b)), para todo $t_{j} \in t_{S_{j}}, \operatorname{com} j=1,2$,

$$
n^{1 / 2}\left[\widehat{S}_{j n}\left(t_{j}\right)-S_{j}\left(t_{j}\right)\right] \stackrel{D}{\longrightarrow} N\left(0, \sigma_{S_{j}}^{2}\right) .
$$


a prova segue pelo lema de Slutsky e o fato de que

$$
\begin{aligned}
& n^{1 / 2}\left(\frac{\partial C_{\alpha}\left(S_{1}\left(t_{1}\right), S_{2}\left(t_{2}\right)\right)}{\partial x}\right) \cdot\left[\widehat{S}_{1 n}\left(t_{1}\right)-S_{1}\left(t_{1}\right)\right] \\
& +n^{1 / 2}\left(\frac{\partial C_{\alpha}\left(S_{1}\left(t_{1}\right), S_{2}\left(t_{2}\right)\right)}{\partial y}\right) \cdot\left[\widehat{S}_{2 n}\left(t_{2}\right)-S_{2}\left(t_{2}\right)\right] \stackrel{D}{\longrightarrow} N\left(0, \sigma_{S}^{2}\right) .
\end{aligned}
$$

\subsubsection{Consistência forte de $\widehat{S}$}

A partir da decomposição dada em (3.2.1) e as convergências quase certas de cada componente, a consistência forte de $\widehat{S}$ é estabelecida no seguinte teorema.

\section{Teorema 3.2.5}

Para os modelos de cópula de Clayton, Estável Positiva e Frank, $\widehat{S}\left(t_{1}, t_{2}\right)=C_{\widehat{\alpha}_{n}}\left(\widehat{S}_{1 n}\left(t_{1}\right), \widehat{S}_{2 n}\left(t_{2}\right)\right)$ é um estimador fortemente consistente de $S\left(t_{1}, t_{2}\right)=C_{\alpha}\left(S_{1}\left(t_{1}\right), S_{2}\left(t_{2}\right)\right)$, para todo $t_{j} \in t_{S_{j}}$, com $j=1,2$.

\section{Prova.}

Com base na decomposição (3.2.1), quando $n \rightarrow \infty$, temos o seguinte para cada componente:

$\circ$ de (3.2.2), $\widehat{\alpha}_{n} \stackrel{\text { q.c. }}{\longrightarrow} \alpha$,

○ do Teorema 3.2.1, $\dot{C}_{a_{n}^{*}}\left(\widehat{S}_{1 n}\left(t_{1}\right), \widehat{S}_{2 n}\left(t_{2}\right)\right) \stackrel{\text { q.c. }}{\longrightarrow} \dot{C}_{\alpha}\left(S_{1}\left(t_{1}\right), S_{2}\left(t_{2}\right)\right)$,

○ e do Corolário 3.2.1, $C_{\alpha}\left(\widehat{S}_{1 n}\left(t_{1}\right), \widehat{S}_{2 n}\left(t_{2}\right)\right) \stackrel{\text { q.c. }}{\longrightarrow} C_{a}\left(S_{1}\left(t_{1}\right), S_{2}\left(t_{2}\right)\right)$.

Assim, considerando que $\dot{C}_{\mathrm{a}}\left(S_{1}\left(t_{1}\right) . S_{2}\left(t_{2}\right)\right.$ ) é limitado (cf. Observação 3.2.2) e que $\left(\widehat{a}_{n}-a\right) \cdot \dot{C}_{n_{n}}\left(\widehat{S}_{1 n}\left(t_{1}\right) . \widehat{S}_{2 n}\left(t_{2}\right)\right) \stackrel{q . c .}{\longrightarrow} 0$. o resultado segue. 


\subsubsection{Convergência fraca de $\widehat{S}$}

O resultado mais importante desta parte, a convergência fraca para o estimador da função de sobrevivência bivariada. $\widehat{S}\left(t_{1}, t_{2}\right)=C_{\widehat{\alpha}_{n}}\left(\widehat{S}_{1 n}\left(t_{1}\right) . \widehat{S}_{2 n}\left(t_{2}\right)\right)$, é estabelecida no seguinte teorema.

\section{Teorema 3.2.6}

Se $0<\sigma_{C}^{2}<\infty$, então para os modelos de cópula de Clayton, Estável Positiva e Frank,

$$
n^{1 / 2}\left[C_{\widehat{\alpha}_{n}}\left(\widehat{S}_{1 n}\left(t_{1}\right), \widehat{S}_{2 n}\left(t_{2}\right)\right)-C_{\alpha}\left(S_{1}\left(t_{1}\right), S_{2}\left(t_{2}\right)\right)\right] \stackrel{D}{\longrightarrow} N\left(0, \sigma_{C}^{2}\right):
$$

quando $n \rightarrow \infty$, para todo $t_{j} \in t_{S_{j}}$, $\operatorname{com} j=1,2$, em que $\sigma_{C}^{2}=\sigma_{a C}^{2}+\sigma_{S}^{2}+2 A$. com $\sigma_{\alpha C}^{2}=\left[\dot{C}_{\alpha}\left(S_{1}\left(t_{1}\right), S_{2}\left(t_{2}\right)\right)\right]^{2} \sigma_{\alpha}^{2}, \sigma_{S}^{2}$ é dado em (3.2.21) e

$$
\begin{aligned}
A=\lim _{n \rightarrow \infty} \operatorname{Cov}\left[\widehat{\alpha}_{n} \cdot \dot{C}_{\alpha_{n}^{*}}\left(\widehat{S}_{1 n}\left(t_{1}\right), \widehat{S}_{2 n}\left(t_{2}\right)\right), C_{\alpha}\left(\widehat{S}_{1 n}\left(t_{1}\right), \widehat{S}_{2 n}\left(t_{2}\right)\right)\right] \\
-\alpha \operatorname{Cov}\left[\dot{C}_{\alpha_{n}^{*}}\left(\widehat{S}_{1 n}\left(t_{1}\right), \widehat{S}_{2 n}\left(t_{2}\right)\right), C_{\alpha}\left(\widehat{S}_{1 n}\left(t_{1}\right), \widehat{S}_{2 n}\left(t_{2}\right)\right)\right]
\end{aligned}
$$

\section{Prova.}

Da decomposição (3.2.1), temos o seguinte, quando $n \rightarrow \infty$ :

○ de (3.2.3), $n^{1 / 2}\left(\widehat{\alpha}_{n}-\alpha\right) \stackrel{D}{\longrightarrow} N\left(0 . \sigma_{\alpha}^{2}\right)$,

o do Teorema (3.2.1), $\dot{C}_{\alpha_{n}^{*}}\left(\widehat{S}_{1 n}\left(t_{1}\right), \widehat{S}_{2 n}\left(t_{2}\right)\right) \stackrel{q . c .}{\longrightarrow} \dot{C}_{\alpha}\left(S_{1}\left(t_{1}\right) . S_{2}\left(t_{2}\right)\right)$,

o e do Teorema (3.2.4),

$$
n^{1 / 2}\left\{C_{a}\left(\widehat{S}_{1 n}\left(t_{1}\right) . \widehat{S}_{2 n}\left(t_{2}\right)\right)-C_{a}\left(S_{1}\left(t_{1}\right) \cdot S_{2}\left(t_{2}\right)\right)\right] \stackrel{D}{\longrightarrow} N\left(0 . \sigma_{s}^{2}\right) .
$$


Como $\dot{C}_{a}\left(S_{1}\left(t_{1}\right), S_{2}\left(t_{2}\right)\right)$ é limitada (cf. Observação 3.2.2), pelo lema de Slutsky,

$$
n^{1 / 2}\left(\widehat{\alpha}_{n}-\alpha\right) \cdot \dot{C}_{\alpha_{n}^{*}}\left(\widehat{S}_{1 n}\left(t_{1}\right), \widehat{S}_{2 n}\left(t_{2}\right)\right) \stackrel{D}{\longrightarrow} N\left(0, \sigma_{\alpha C}^{2}\right),
$$

$\operatorname{com} \sigma_{\alpha C}^{2}=\left[\dot{C}_{\alpha}\left(S_{1}\left(t_{1}\right), S_{2}\left(t_{2}\right)\right)\right]^{2} \sigma_{\alpha}^{2}$.

Assim,

$$
n^{1 / 2}\left[C_{\widehat{\alpha}_{n}}\left(\widehat{S}_{1 n}\left(t_{1}\right), \widehat{S}_{2 n}\left(t_{2}\right)\right)-C_{\alpha}\left(S_{1}\left(t_{1}\right), S_{2}\left(t_{2}\right)\right)\right] \stackrel{D}{\longrightarrow} N\left(0, \sigma_{C}^{2}\right) .
$$

Concluímos esta seção destacando que, como mencionado em Wang e Wells (2000), a variância assintótica de $C_{\widehat{\alpha}_{n}}\left(\widehat{S}_{1 n}, \widehat{S}_{2 n}\right)$ é uma expressão complicada de tratar analiticamente.

Dentro de uma perspectiva Bayesiana, considerando a função de sobrevivência bivariada como uma função da distribuição a posteriori, estimativas de Monte Carlo podem ser calculadas para essa variância. Uma outra alternativa é considerar métodos baseados em re-amostragem, como por exemplo usar o método Bootstrap (veja Efron, 1982), para obter uma estimativa da variância assintótica.

Avaliar o desempenho destes dois métodos de estimação da variância assintótica é um tópico de pesquisa futura. 


\subsubsection{Prova Proposições 3.2.1, 3.2.2 e parte (i) Teore- ma 3.2.3(a)}

\section{Prova Proposição 3.2.1}

Pelo Teorema B.1, para mostrar eqüicontinuidade de $\dot{C}_{\left(\alpha_{n}^{*}\right)}(x, y) \in E$ em $\alpha$ para os três modelos considerados basta mostrar que o $\lim \dot{C}_{\left(\alpha_{n}^{*}\right)}(x, y)=$ $\dot{C}_{(\alpha)}(x, y)$, para toda seqüência $\alpha_{n}^{*} \in \mathcal{A} \operatorname{com} \lim \alpha_{n}^{*}=\alpha$, quando $n \rightarrow \infty$.

(a) Para a cópula de Clayton, (3.2.6) pode ser escrita em termos de $C_{\alpha}(x, y)$ por,

$$
\begin{aligned}
\dot{C}_{\left(\alpha_{n}^{*}\right)}(x, y)= & \alpha_{n}^{*-1}\left[\left(x^{-\alpha_{n}^{*}} \ln x+y^{-\alpha_{n}^{*}} \ln y\right) C_{\alpha_{n}^{*}}(x, y)^{\alpha_{n}^{*}+1}\right. \\
& \left.-C_{\alpha_{n}^{*}}(x, y) \ln C_{\alpha_{n}^{*}}(x, y)\right] .
\end{aligned}
$$

No que segue, considere cada $\omega \in B$, com $\operatorname{Pr}\{B\}=1$. Defina $f_{1}$ : $\mathbb{R}^{+} \mapsto \mathbb{R}^{+}$; por $f_{1}\left(\alpha_{n}^{*}(\omega)\right)=\alpha_{n}^{*}(\omega)^{-1}, f_{2}: \mathbb{R}^{+} \mapsto \mathbb{R}^{-}$, por $f_{2}\left(\alpha_{n}^{*}(\omega)\right)=$ $x^{-\alpha_{n}^{*}(\omega)} \ln x+y^{-\alpha_{n}^{*}(\omega)} \ln y$, e $f_{3}: \mathbb{R}^{+} \mapsto \mathbb{R}^{+}$, por $f_{3}\left(\alpha_{n}^{*}(\omega)\right)=C_{\alpha_{n}^{*}(\omega)}(x, y)$. Logo, $\lim _{n \rightarrow \infty} f_{1}\left(\alpha_{n}^{*}(\omega)\right)=\alpha^{-1}, \operatorname{com} \alpha \in \mathbb{R}^{+}$, para todo $\omega \in B$.

Também, para todo $\omega \in B, \lim _{n \rightarrow \infty} f_{2}\left(\alpha_{n}^{*}(\omega)\right)=x^{-\alpha} \ln x+y^{-\alpha} \ln y$, para todo $x \in\left[S_{1}\left(\tau_{1}\right), 1\right]$ e $y \in\left[S_{2}\left(\tau_{2}\right), 1\right]$, pois,

$$
\begin{aligned}
\lim _{n \rightarrow \infty}\left[f_{2}\left(\alpha_{n}^{*}(\omega)\right)-f_{2}(\alpha)\right]= & \lim _{n \rightarrow \infty}\left(x^{-\alpha_{n}^{*}(\omega)} \ln x+y^{-\alpha_{n}^{*}(\omega)} \ln y\right. \\
& \left.-x^{-\alpha} \ln x-y^{-\alpha} \ln y\right) \\
= & \ln x \lim _{n \rightarrow \infty}\left[x^{-\alpha_{n}^{*}(\omega)+\alpha} x^{\alpha_{n}^{*}(\omega)}\left(x^{a-\alpha_{n}^{*}(\omega)}-1\right)\right] \\
& +\ln y \lim _{n \rightarrow \infty}\left[y^{-\alpha_{n}^{*}(\omega)+\alpha} y^{\alpha_{n}^{*}(\omega)}\left(y^{\alpha-\alpha_{n}^{*}(\omega)}-1\right)\right] \\
= & 0 .
\end{aligned}
$$

Do mesmo modo. para todo $\omega^{\prime} \in B . \lim _{n-x} f_{3}\left(\alpha_{n}^{*}\left(\omega^{\prime}\right)\right)=C_{a}(x, y)$. para 
todo $x \in\left[S_{1}\left(\tau_{1}\right), 1\right]$ e $y \in\left[S_{2}\left(\tau_{2}\right), 1\right]$, pois,

$$
\begin{aligned}
\lim _{n \rightarrow \infty}\left[f_{3}\left(\alpha_{n}^{*}(\omega)\right)-f_{3}(\alpha)\right]= & \lim _{n \rightarrow \infty}\left(w_{\alpha_{n}^{*}(\omega)}^{-1 / \alpha_{n}^{*}(\omega)}-w_{\alpha}^{-1 / a}\right) \\
= & \lim _{n \rightarrow \infty}\left\{w_{\alpha_{n}^{*}(\omega)}^{-1 / \alpha}\left[w_{\alpha_{n}^{*}(\omega)}^{\frac{\alpha_{n}^{*}(\omega)-\alpha}{\alpha+(\omega)}}-\left(\frac{w_{\alpha}}{w_{\alpha_{n}^{*}(\omega)}}\right)^{-1 / \alpha}\right]\right\} \\
= & \lim _{n \rightarrow \infty}\left\{w _ { \alpha _ { n } ^ { * } ( \omega ) } ^ { - 1 / \alpha } \left[w_{\alpha_{n}^{*}(\omega)}^{\frac{\alpha_{n}^{*}(\omega)-\alpha}{\alpha \alpha_{n}^{*}(\omega)}}-\left((x y)^{\alpha_{n}^{*}(\omega)-\alpha}\right.\right.\right. \\
& \left.\left.\left.\cdot \frac{x^{\alpha}+y^{\alpha}-(x y)^{\alpha}}{x^{\alpha_{n}^{*}(\omega)}+y^{\alpha_{n}^{*}(\omega)}-(x y)^{\alpha_{n}^{*}(\omega)}}\right)^{-1 / \alpha}\right]\right\} \\
= & 0 .
\end{aligned}
$$

Assim, (3.2.22) pode ser escrita como um produto, diferença e composição de funções contínuas em $\alpha$, e portanto, pelos Teoremas B.2 e B.3, corresponde a uma função contínua em $\alpha$, para todo $x \in\left[S_{1}\left(\tau_{1}\right), 1\right]$ e $y \in\left[S_{2}\left(\tau_{2}\right), 1\right]$, e assim, para todo $\omega \in B$ com $\operatorname{Pr}\{B\}=1$,

$$
\begin{aligned}
\lim _{n \rightarrow \infty} \dot{C}_{\left(\alpha_{n}^{*}(\omega)\right)}(x, y) & =f_{1}(\alpha)\left[f_{2}(\alpha) f_{3}(\alpha)^{\alpha+1}-f_{3}(\alpha) \ln f_{3}(\alpha)\right] \\
& =\dot{C}_{(\alpha)}(x, y) .
\end{aligned}
$$

Logo, para a cópula de Clayton a função $\dot{C}_{\left(\alpha_{n}^{*}(\omega)\right)}(x, y) \in E$ é eqüicontínua em $\alpha$, para todo $\omega \in B$ com $\operatorname{Pr}\{B\}=1$.

(b) Para a cópula Estável Positiva, (3.2.7) pode ser escrita em termos de $C_{\alpha}(x, y)$ por;

$$
\begin{aligned}
\dot{C}_{\left(\alpha_{n}^{*}\right)}(x, y)= & \alpha_{n}^{*-2} C_{\alpha_{n}^{*}}(x, y)\left[-\ln C_{\alpha_{n}^{*}}(x, y)\right]^{1-1 / \alpha_{n}^{*}} \ln \left[-\ln C_{\alpha_{n}^{*}}(x, y)\right] \\
& \cdot\left[(-\ln x)^{1 / \alpha_{n}^{*}} \ln (-\ln x)+(-\ln y)^{1 / \alpha_{n}^{*}} \ln (-\ln y)\right] .
\end{aligned}
$$

Considere para cada $\omega^{\prime} \in B \operatorname{com} \operatorname{Pr}\{B\}=1$ o seguinte. Defina $f_{1}$ : $\mathbb{R}^{+} \mapsto \mathbb{R}^{+} \cdot$ por $f_{1}\left(a_{n}^{*}(\omega)\right)=a_{n}^{*}(\omega)^{-2} \cdot f_{2}: \mathbb{R}^{+} \mapsto \mathbb{R}^{+}$. por $f_{2}\left(a_{n}^{*}(\omega)\right)=$ 
$\left[(-\ln x)^{1 / \alpha_{n}^{*}(\omega)}+(-\ln y)^{1 / \alpha_{n}^{*}(\omega)}\right]^{\alpha_{n}^{*}(\omega)}$, e $f_{3}: \mathbb{R}^{+} \mapsto \mathbb{R}$; por $f_{3}\left(\alpha_{n}^{*}(\omega)\right)=$ $(-\ln x)^{1 / a_{n}^{*}(\omega)} \ln (-\ln x)+(-\ln y)^{1 / a_{n}^{*}(\omega)} \ln (-\ln y)$.

Então, $\lim _{n \rightarrow \infty} f_{1}\left(\alpha_{n}^{*}(\omega)\right)=\alpha^{-2}$, com $\alpha \in(0,1)$, para todo $\omega \in B$.

Também; para todo $\omega \in B, \lim _{n \rightarrow \infty} f_{2}\left(\alpha_{n}^{*}(\omega)\right)=\left[(-\ln x)^{1 / \alpha}+\right.$ $\left.(-\ln y)^{1 / \alpha}\right]^{\alpha}$, para todo $x \in\left[S_{1}\left(\tau_{1}\right), 1\right]$ e $y \in\left[S_{2}\left(\tau_{2}\right), 1\right]$, pois,

$$
\begin{aligned}
\lim _{n \rightarrow \infty}\left[f_{2}\left(\alpha_{n}^{*}(\omega)\right)-f_{2}(\alpha)\right]= & \lim _{n \rightarrow \infty}\left\{\left[(-\ln x)^{1 / \alpha_{n}^{*}(\omega)}+(-\ln y)^{1 / \alpha_{n}^{*}(\omega)}\right]_{n}^{*}(\omega)\right. \\
& \left.-\left[(-\ln x)^{1 / \alpha}+(-\ln y)^{1 / \alpha}\right]^{\alpha}\right\} \\
= & 0 .
\end{aligned}
$$

Do mesmo modo, para todo $\omega \in B$, $\lim _{n \rightarrow \infty} f_{3}\left(\alpha_{n}^{*}(\omega)\right)=$ $(-\ln x)^{1 / \alpha} \ln (-\ln x)+(-\ln y)^{1 / \alpha} \ln (-\ln y)$, para todo $x \in\left[S_{1}\left(\tau_{1}\right), 1\right]$ e $y \in\left[S_{2}\left(\tau_{2}\right), 1\right]$, pois,

$$
\begin{aligned}
\lim _{n \rightarrow \infty}\left[f_{3}\left(\alpha_{n}^{*}(\omega)\right)-f_{3}(\alpha)\right]= & \lim _{n \rightarrow \infty}\left[(-\ln x)^{1 / \alpha_{n}^{*}(\omega)} \ln (-\ln x)\right. \\
& +(-\ln y)^{1 / \alpha_{n}^{*}(\omega)} \ln (-\ln y) \\
& \left.-(-\ln x)^{1 / \alpha} \ln (-\ln x)-(-\ln y)^{1 / \alpha} \ln (-\ln y)\right] \\
= & 0 .
\end{aligned}
$$

Assim, (3.2.23) pode ser escrita como produto e composição de funções contínuas em $\alpha$, que corresponde também a uma função contínua em $\alpha$ para todo $x \in\left[S_{1}\left(\tau_{1}\right), 1\right]$ e $y \in\left[S_{2}\left(\tau_{2}\right), 1\right]$, i.e., para todo $\omega \in B$ com $\operatorname{Pr}\{B\}=1$,

$$
\begin{aligned}
\lim _{n \rightarrow x} \dot{C}_{\left(a_{n}^{*}(w)\right)}(x, y) & =f_{1}(\alpha) \exp \left\{-f_{2}(\alpha)\right\} f_{2}(a)^{1-1 / \alpha} \ln \left(f_{2}(\alpha)\right) f_{3}(a) \\
& =\dot{C}_{(a)}(x, y)
\end{aligned}
$$

Logo. para a cópula Estável Positiva. a função $\dot{C}_{\left(\alpha_{n}^{*}\left(\omega^{*}\right)\right)}(x, y) \in E$ é equïicontínua em $a$. para torlo $\omega \in B \operatorname{com} \operatorname{Pr}\{B\}=1$. 
(c) Para a cópula de Frank, (3.2.8) pode ser escrita em termos de $C_{\alpha}(x, y)$ por,

$$
\begin{aligned}
& \dot{C}_{\left(\alpha_{n}^{*}\right)}(x, y)=\frac{\alpha_{n}^{*-1}}{\ln \alpha_{n}^{*}} {\left[x+y-\frac{\alpha_{n}^{*}}{\alpha_{n}^{*}-1}+\frac{x \alpha_{n}^{*}+y}{y \alpha_{n}^{* x}-\alpha_{n}^{*}(x+y-1)} r_{\alpha_{n}^{*}}\right.} \\
&\left.-C_{\alpha_{n}^{*}}(x, y)\right] .
\end{aligned}
$$

Considere para cada $\omega \in B$ com $\operatorname{Pr}\{B\}=1$ o seguinte. Defina $f_{1}$ : $\mathbb{R}^{+} \mapsto \mathbb{R}^{+}$, por $f_{1}\left(\alpha_{n}^{*}(\omega)\right)=\alpha_{n}^{*}(\omega)^{-1}, f_{2}: \mathbb{R}^{+} \mapsto \mathbb{R}$, por $f_{2}\left(\alpha_{n}^{*}(\omega)\right)=$ $x \alpha_{n}^{*}(\omega)^{y}+y \alpha_{n}^{*}(\omega)^{x}-\alpha_{n}^{*}(\omega)(x+y-1)$, e $f_{3}: \mathbb{R}^{+} \mapsto \mathbb{R}^{+}$; por $f_{3}\left(\alpha_{n}^{*}(\omega)\right)=$ $\alpha_{n}^{*}(\omega)-1+\left(\alpha_{n}^{*}(\omega)^{y}-1\right)\left(\alpha_{n}^{*}(\omega)^{x}-1\right)$.

Então, $\lim _{n \rightarrow \infty} f_{1}\left(\alpha_{n}^{*}(\omega)\right)=\alpha^{-1}$; com $\alpha \in(0,1)$, para todo $\omega \in B$.

Também, para todo $\omega \in B, \lim _{n \rightarrow \infty} f_{2}\left(\alpha_{n}^{*}(\omega)\right)=x \alpha^{y}+y \alpha^{x}-\alpha(x+y-1)$, para todo $x \in\left[S_{1}\left(\tau_{1}\right), 1\right]$ e $y \in\left[S_{2}\left(\tau_{2}\right), 1\right]$, pois;

$$
\begin{aligned}
\lim _{n \rightarrow \infty}\left[f_{2}\left(\alpha_{n}^{*}(\omega)\right)-f_{2}(\alpha)\right]= & \lim _{n \rightarrow \infty}\left\{x \alpha_{n}^{*}(\omega)^{y}+y \alpha_{n}^{*}(\omega)^{x}-\alpha_{n}^{*}(\omega)(x+y-1)\right. \\
& \left.-x \alpha^{y}-y \alpha^{x}+\alpha(x+y-1)\right\} \\
= & \lim _{n \rightarrow \infty}\left\{x \alpha^{y}\left[\left(\alpha_{n}^{*}(\omega) \alpha\right)^{y}-1\right]+y \alpha^{x}\left[\left(\alpha_{n}^{*}(\omega) / \alpha\right)^{x}-1\right]\right. \\
& \left.-(x+y-1)\left(\alpha_{n}^{*}(\omega)-\alpha\right)\right\} \\
= & 0 .
\end{aligned}
$$

Agora, para todo $\omega \in B, \lim _{n \rightarrow \infty} f_{3}\left(\alpha_{n}^{*}(\omega)\right)=\alpha-1+\left(\alpha^{y}-1\right)\left(\alpha^{x}-1\right)$, para todo $x \in\left[S_{1}\left(\tau_{1}\right), 1\right]$ e $y \in\left[S_{2}\left(\tau_{2}\right), 1\right]$, pois;

$$
\begin{aligned}
\lim _{n \rightarrow \infty}\left[f_{3}\left(\alpha_{n}^{*}(\omega)\right)-f_{3}(\alpha)\right]= & \lim _{n \rightarrow \infty}\left[\alpha_{n}^{*}(\omega)+\left(\alpha_{n}^{*}(\omega)^{y}-1\right)\left(\alpha_{n}^{*}(\omega)^{x}-1\right)\right. \\
& \left.-\alpha-\left(\alpha^{y}-1\right)\left(\alpha^{x}-1\right)\right] \\
= & \lim _{n \rightarrow \infty}\left\{\alpha_{n}^{*}(\omega)-\alpha+\alpha^{x+y}\left[\left(\alpha_{n}^{*}(\omega) / \alpha\right)^{x+y}-1\right]\right. \\
& \left.-\alpha^{x}\left[\left(\alpha_{n}^{*}(\omega) / \alpha\right)^{x}-1\right]-\alpha^{y}\left[\left(\alpha_{n}^{*}(\omega) / \alpha\right)^{y}-1\right]\right\} \\
= & 0 .
\end{aligned}
$$


Assim: (3.2.24) pode ser escrita como produto, soma, diferença e composição de funções contínuas em $\alpha$, o que corresponde a uma função contínua em $a$ para todo $x \in\left[S_{1}\left(\tau_{1}\right), 1\right]$ e $y \in\left[S_{2}\left(\tau_{2}\right)\right.$, 1], i.e., para todo $\omega \in B$ com $\operatorname{Pr}\{B\}=1$,

$$
\begin{aligned}
\lim _{n \rightarrow \infty} \dot{C}_{\left(\alpha_{n}^{*}(w)\right)}(x, y)= & \frac{f_{1}(\alpha)}{-\ln f_{1}(\alpha)}\left[x+y-\frac{1}{1-f_{1}(\alpha)}+\frac{f_{2}(\alpha)}{f_{3}(\alpha)}\right. \\
& \left.+\frac{1}{\ln f_{1}(\alpha)} \ln \left(\frac{f_{3}(\alpha)}{f_{1}^{-1}(\alpha)-1}\right)\right] \\
= & \dot{C}_{(\alpha)}(x, y) .
\end{aligned}
$$

Logo, para a cópula de Frank, a função $\dot{C}_{\left(\alpha_{n}^{*}\right)}(x, y) \in E$ é eqüicontínua em $\alpha$, para todo $\omega \in B$ com $\operatorname{Pr}\{B\}=1$.

\section{Prova Proposição 3.2.2}

Primeiro note que para as três cópulas consideradas, nas expressões de $\dot{C}_{\alpha}(\cdot, \cdot)$, dadas em (3.2.22), (3.2.23) e (3.2.24), o termo $C_{\alpha}(x, y)$ é uma função uniformemente contínua em $[0,1]^{2}$ (veja Nelsen, 1999).

Para a cópula de Clayton, note que $f(x, y)=x^{-\alpha} \ln x+y^{-\alpha} \ln y$, é contínua para todo $(x, y) \in\left[S_{1}\left(\tau_{1}\right), 1\right] \times\left[S_{2}\left(\tau_{2}\right), 1\right]$.

Assim, (3.2.22) é escrita como produto, diferença e composição de funções contínuas e corresponde a uma função contínua para todo $x \in\left[S_{1}\left(\tau_{1}\right), 1\right]$ e $y \in\left[S_{2}\left(\tau_{2}\right), 1\right]$.

No caso da cópula Estável Positiva note que $f(x \cdot y)=(-\ln x)^{1 / a} \ln (-\ln x)+$ $(-\ln y)^{1 / \alpha} \ln (-\ln y)$. é contínua para todo $(x \cdot y) \in\left[S_{1}\left(\tau_{1}\right) \cdot 1\right] \times\left[S_{2}\left(\tau_{2}\right) .1\right]$. Assim. (3.2.23) pode ser escrita como produto e composição de funções contínuas. que corresponde a uma função contínua para todo $x \in\left[S_{1}\left(\tau_{1}\right) .1\right]$ $e y \in\left[S_{2}\left(\tau_{2}\right) \cdot 1\right]$. 
Finalmente, para a cópula de Frank note que $f(x, y)=(x+y)+$ $r_{\alpha}^{-1}\left[x \alpha^{y}+y \alpha^{x}-\alpha(x+y-1)\right]$, que também é contínua para todo $(x, y) \in\left[S_{1}\left(\tau_{1}\right), 1\right] \times\left[S_{2}\left(\tau_{2}\right), 1\right]$.

Logo, (3.2.24) também pode ser expressa como soma e diferença de funções contínuas, e assim, é uma função contínua para todo $x \in\left[S_{1}\left(\tau_{1}\right), 1\right]$ e $y \in\left[S_{2}\left(\tau_{2}\right), 1\right]$.

Portanto, para os três modelos de cópula considerados, $\dot{C}_{\alpha}(\cdot, \cdot)$ sendo definida sobre o compacto $\left[S_{1}\left(\tau_{1}\right), 1\right] \times\left[S_{2}\left(\tau_{2}\right), 1\right]$, segue que pelo Teorema B.j é uniformemente contínua.

\section{Prova parte (i) Teorema 3.2.3(a)}

Seja $C_{\alpha}(u, v)=\operatorname{Pr}\{U \leq u, V \leq v\}$, para $0 \leq u, v \leq 1$, com $U, V \sim U(0,1)$, e $\alpha \in \mathcal{A}$. Considere para $c_{\alpha}(u, v)=\frac{\partial^{2} C_{\alpha}(u, v)}{\partial u \partial v}$,

$$
\begin{aligned}
& C_{\alpha}(u, v)=\int_{0}^{u} \int_{0}^{v} c_{\alpha}(s, t) d s d t, \\
& C_{\alpha}(u, 1)=\int_{0}^{u} \int_{0}^{1} c_{\alpha}(s, t) d s d t=u=S_{1}\left(t_{1}\right), \text { para } t_{1} \in t_{S_{1}} \mathrm{e} \\
& C_{\alpha}(1, v)=\int_{0}^{v} \int_{0}^{1} c_{\alpha}(s, t) d s d t=v=S_{2}\left(t_{2}\right), \text { para } t_{2} \in t_{S_{2}} .
\end{aligned}
$$

Defina os seguintes conjuntos de funções,

$$
\begin{aligned}
& \Lambda=\left\{c_{\alpha}:[0,1]^{2} \mapsto[0,1] ; 0 \leq c_{\alpha} \leq 1: \iint c_{\alpha}<\infty ; c_{\alpha} \in C\left([0,1]^{2}\right), \alpha \in \mathcal{A}\right\}, \\
& \Gamma_{1}=\left\{C_{\alpha}:[0,1]^{2} \mapsto[0.1] ; 0 \leq C_{\alpha} \leq 1, C_{\alpha}(u, v)=\int_{0}^{u} \int_{0}^{v} c_{\alpha}(s, t) d l \cdot s d t, c_{\alpha} \in A\right\} \text {, } \\
& \Gamma_{2}=\left\{u=S_{1}\left(t_{1}\right): 0<u<1 ; u=\int_{0}^{u} \int_{0}^{1} c_{0}(s . t) d . s d t . c_{\alpha} \in A . t_{1} \in t_{S_{1}}\right\} . \\
& \Gamma_{3}=\left\{v=S_{2}\left(t_{2}\right): 0<v^{\prime}<1: v=\int_{0}^{v} \int_{0}^{1} c_{\alpha}(s, t) d s d t . c_{\alpha} \in 1 . t_{2} \in t_{S_{2}}\right\} .
\end{aligned}
$$


Note que $\Gamma_{1} \subset C\left([0,1]^{2}\right)$ e $\Gamma_{i} \subset C([0,1]), j=2.3$. Definamos também a norma uniforme, i.e., para $C_{\alpha} \in \Gamma_{1},\left\|C_{\alpha}\right\|_{\Gamma 1}=\sup _{(u, v) \in[0,1]^{2}}\left|C_{\alpha}(u . v)\right|$ : para $u \in \Gamma_{2}, \quad\|u\|_{\Gamma_{2}}=\sup _{u \in[0,1]}\left|C_{\alpha}(u, 1)\right|$, e para $v \in \Gamma_{3}$, $\|v\|_{\Gamma_{3}}=\sup _{v \in[0,1]}\left|C_{\alpha}(1, v)\right|$.

Então, defina a aplicação $\xi: C\left([0,1]^{2}\right) \mapsto C\left([0,1]^{2}\right) \times C([0,1]) \times C([0,1])$, tal que

$$
\xi\left(C_{\alpha}\right)=\left(\xi_{1}\left(C_{\alpha}\right), \xi_{2}\left(C_{\alpha}\right), \xi_{3}\left(C_{\alpha}\right)\right)
$$

com

$$
\begin{aligned}
& \xi_{1}\left(C_{\alpha}\right)(u, v)=\left(\pi_{1} \circ \xi\right)\left(C_{\alpha}\right)=C_{\alpha}(u, v), \\
& \xi_{2}\left(C_{\alpha}\right)(u, v)=\left(\pi_{2} \circ \xi\right)\left(C_{\alpha}\right)=u, \\
& \xi_{3}\left(C_{\alpha}\right)(u, v)=\left(\pi_{3} \circ \xi\right)\left(C_{\alpha}\right)=v .
\end{aligned}
$$

Note que $\xi_{1}: \Gamma_{1} \rightarrow \Gamma_{1}$ é a função identidade (função linear e contínua), $\xi_{2}: \Gamma_{1} \rightarrow \Gamma_{2}$ e $\xi_{3}: \Gamma_{1} \rightarrow \Gamma_{3}$.

Para provar linearidade de $\xi$ provaremos que para $a, b \in \mathbb{R}^{+}$,

$$
\xi\left(a C_{\alpha}+b C_{1 \alpha}\right)=a \xi\left(C_{\alpha}\right)+b \xi\left(C_{1 \alpha}\right),
$$

$\operatorname{com} C_{\alpha}, C_{1 \alpha} \in \Gamma_{1}$. De fato,

$$
\begin{aligned}
\xi\left(a C_{\alpha}+b C_{1 \alpha}\right) & =\left(\xi_{1}\left(a C_{\alpha}+b C_{1 \alpha}\right), \xi_{2}\left(a C_{\alpha}+b C_{1 \alpha}\right): \xi_{3}\left(a C_{\alpha}+b C_{1 \alpha}\right)\right) \\
& =\left(a C_{\alpha}+b C_{1 \alpha}, \xi_{2}\left(a C_{\alpha}+b C_{1 \alpha}\right): \xi_{3}\left(a C_{\alpha}+b C_{1 \alpha}\right)\right) .
\end{aligned}
$$

Seja $Z=\xi_{2}\left(a C_{0}+b C_{1 \alpha}\right)$. Note que 


$$
\begin{aligned}
\left(a C_{\alpha}+b C_{1 \alpha}\right)(u, v) & =a C_{\alpha}(u, v)+b C_{1 \alpha}(u, v) \\
& =a \int_{0}^{u} \int_{0}^{v} c_{\alpha}(s, t) d s d t+b \int_{0}^{u} \int_{0}^{v} c_{1 \alpha}(s, t) d s d t, \quad c_{\alpha}, c_{1 \alpha} \in \Lambda, \\
& =\int_{0}^{u} \int_{0}^{v}\left[a c_{\alpha}(s, t)+b c_{1 \alpha}(s, t)\right] d s d t, \text { com } a c_{\alpha}+b c_{1 \alpha} \in \Lambda .
\end{aligned}
$$

Logo, $a C_{\alpha}+b C_{1 \alpha} \in \Gamma_{1}$.

Agora, considere

$$
\begin{aligned}
Z(u) & =\left(a C_{\alpha}+b C_{1 \alpha}\right)(u, 1) \\
& =\int_{0}^{u} \int_{0}^{1}\left[a c_{\alpha}(s, t)+b c_{1 \alpha}(s, t)\right] d t d s \\
& =a \int_{0}^{u} \int_{0}^{1} c_{\alpha}(s, t) d t d s+b \int_{0}^{u} \int_{0}^{1} c_{1 \alpha}(s, t) d t d s \\
& =a C_{\alpha}(u, 1)+b C_{1 \alpha}(u, 1) \\
& =a u+b u \\
& =a \xi_{2}\left(C_{\alpha}\right)(u, v)+b \xi_{2}\left(C_{1 \alpha}\right)(u, v)
\end{aligned}
$$

Então, $Z=a \xi_{2}\left(C_{\alpha}\right)+b \xi_{2}\left(C_{1 \alpha}\right)$. Mas $Z=\xi_{2}\left(a C_{\alpha}+b C_{1 \alpha}\right)$, e assim $\xi_{2}\left(a C_{\alpha}+\right.$ $\left.b C_{1 \alpha}\right)=a \xi_{2}\left(C_{\alpha}\right)+b \xi_{2}\left(C_{1 \alpha}\right)$, o que mostra que $\xi_{2}$ é linear.

Analogamente, $\xi_{3}$ também é linear. Logo, substituindo em (3.2.25),

$$
\begin{aligned}
\xi\left(a C_{a}+b C_{1 \alpha}\right) & =\left(a C_{\alpha}+b C_{1 \alpha}: \xi_{2}\left(a C_{\alpha}+b C_{1 \alpha}\right), \xi_{3}\left(a C_{\alpha}+b C_{1 \alpha}\right)\right) \\
& =\left(a C_{\alpha}+b C_{1 \alpha}: a \xi_{2}\left(C_{\alpha}\right)+b \xi_{2}\left(C_{1 \alpha}\right), a \xi_{3}\left(C_{\alpha}\right)+b \xi_{3}\left(C_{1 \alpha}\right)\right) \\
& =a\left(C_{\alpha}, \xi_{2}\left(C_{\alpha}\right), \xi_{3}\left(C_{\alpha}\right)\right)+b\left(C_{1 \alpha}, \xi_{2}\left(C_{1 \alpha}\right), \xi_{3}\left(C_{1 \alpha}\right)\right) \\
& =a \xi\left(C_{\alpha}\right)+b \xi\left(C_{1 \alpha}\right) .
\end{aligned}
$$


Para provar continuidade de $\xi$, dado que $\xi$ é linear, basta mostrar contimuidade no zero. Provaremos para $\xi_{2}$ que,

$$
\forall \varepsilon>0, \exists \delta>0, \text { tal que }\left\|C_{\alpha}\right\|_{\Gamma_{1}}<\delta \Rightarrow\left\|\xi_{2}\left(C_{\alpha}\right)\right\|_{\Gamma_{2}}=\|u\|_{\Gamma_{2}}<\Xi .
$$

De fato,

$$
\left\|C_{\alpha}\right\|_{\Gamma_{1}}=\sup _{(u, v) \in[0,1]^{2}}\left|\int_{0}^{u} \int_{0}^{v} c_{\alpha}(s, t) d s d t\right|=\iint_{[0,1]^{2}} c_{\alpha}(s, t) d s d t,
$$

e

$$
\|u\|_{\Gamma_{2}}=\sup _{u \in[0,1]}\left|\int_{0}^{u} \int_{0}^{1} c_{\alpha}(s, t) d s d t\right|=\iint_{[0,1]^{2}} c_{\alpha}(s, t) d s d t .
$$

Assim, $\left\|C_{\alpha}\right\|_{\Gamma_{1}}=\|u\|_{\Gamma_{2}}$, e dado $\varepsilon>0$ considerando $\delta=\varepsilon$, temos que $\left\|C_{\alpha}\right\|_{\Gamma_{1}}<\delta \Rightarrow\|u\|_{\Gamma_{2}}<\varepsilon$. Logo, $\xi_{2}$ é contínua no zero e dado que também é linear, $\xi_{2}$ é contínua em todo ponto de $\Gamma_{2}$.

Analogamente, $\xi_{3}$ é contínua em todo ponto de $\Gamma_{3}$.

Dado que $\xi_{1}, \xi_{2}$ e $\xi_{3}$ são aplicaçōes contínuas, i.e., $\left(\pi_{1} \circ \xi\right),\left(\pi_{2} \circ \xi\right)$ e $\left(\pi_{3} \circ \xi\right)$ são contínuas, temos que $\xi$ é uma aplicação contínua.

Portanto, a aplicação $C_{\alpha} \mapsto\left(C_{\alpha}, u, v\right)$ é linear e contínua. 


\section{Capítulo 4}

\section{Considerações finais e perspectivas de trabalhos \\ futuros}

Neste trabalho consideramos uma abordagem Bayesiana paramétrica para o estudo de dados de sobrevivência bivariados. A estrutura de dependência entre os tempos de falha foi modelada através de cópulas Arquimedianas e procedimentos Bayesianos de estimação (em duas etapas e conjunta) foram propostos. No Capítulo 3, considerando a cópula como um funcional das funções de sobrevivência marginais, foram estudadas a convergência forte e a convergência fraca do estimador proposto para a função de sobrevivência bivariada. Foram utilizados argumentos de convergência de estimadores obtidos por métodos de estimação via MC\IC, de continuidade e o método delta para funcionais.

Destacamos também que o enfoque baseado em cópulas, além de ser flexível 
com respeito à escolha das distribuições marginais, nos permitiu obter o Teorema Central do Limite para funcionais no estudo das propriedades assintóticas do estimador da função de sobrevivência bivariada.

Notamos que, a relação entre os modelos de sobrevivência com fragilidade multiplicativa e a família de cópulas Arquimedianas, conduz a uma interpretação da função de razões cruzadas como uma medida de dependência local. Além disso, a ligação entre o coeficiente tau de Kendall e cópulas, nos permite quantificar a dependência global entre os tempos de falha.

Como já foi dito no Capítulo anterior, e como também é mencionado em Dabrowska (1989), mas em um contexto clássico completamente não paramétrico, a variância assintótica do estimador da função de sobrevivência bivariada $C_{\widehat{\alpha}_{n}}\left(\widehat{S}_{1 n}, \widehat{S}_{2 n}\right)$, é uma expressão complicada de ser tratada analiticamente. Estimativas de Monte Carlo podem ser calculadas para essa variância considerando a função de sobrevivência bivariada como uma função da distribuição a posteriori. Pode ser de interesse avaliar o desempenho deste método de estimação da variância assintótica com outros métodos baseados em re-amostragem (Bootstrap ou Jackknife), como sugerido em Dabrowska (1989).

Neste trabalho analisamos em detalhes três modelos de cópulas Arquimedianas: Clayton. Estável Positiva e Frank. Um modelo a ser pesquisado é o modelo de cópula induzido pela distribuição de fragilidade Poisson. Como foi mencionado nas aplicações, ao observar as curvas de Kaplan- Keier, alguns indivíduos que receberam tratamento poderiam constituir uma proporção de sujeitos curados. Segundo Oakes (1989). o modelo de fragilidade Poisson 
está relacionado com modelos de sobrevivência com taxa de cura (veja por exemplo Maller e Zhou, 1996).

Um outro modelo a ser pesquisado é o modelo de cópula obtido a partir da distribuição de fragilidade PVF (power variance function) que inclui como casos particulares as distribuições Gama, Estável Positiva e Gaussiana Inversa (veja Hougaard, 2000, Seção 7.5).

Finalmente, planejamos desenvolver uma biblioteca no pacote $\mathrm{R}$ para modelar dados bivariados em Análise de Sobrevivência com a metodologia descrita neste trabalho. Além da modelagem dos dados pretendemos disponibilizar critérios de comparação de modelos como aqueles apresentados neste trabalho. Com base nesta biblioteca avaliaremos o desempenho de diferentes critérios de comparação de modelos, aplicando-os tanto a dados da literatura como a dados simulados. Dentro dos critérios a serem pesquisados estudaremos a estatística $D$, utilizada na Seção 2.4.2, a estatística $A P L M L$ calculada com base no critério CPO (veja Ibrahim et al., 2001, Seção 6.3), e o Critério de Informação Bayesiano BIC. 


\section{Apêndice A}

\section{Método Delta para Funcionais}

Uma importante ferramenta em Teoria Assintótica é o Método Delta: Suponha $X_{n}$ uma sequência de variáveis aleatórias com $n^{1 / 2}\left(X_{n}-\theta\right) \stackrel{D}{\longrightarrow} Z$ para alguma constante $\theta$; quando $n \rightarrow \infty$. Se $\Psi: \mathbb{R} \mapsto \mathbb{R}$ é diferenciável em $\theta$, então pelo lema de Slutsky,

$$
n^{1 / 2}\left[\Psi\left(X_{n}\right)-\Psi(\theta)\right] \stackrel{D}{\longrightarrow} \Psi^{\prime}(\theta) \cdot Z,
$$

e de fato, $n^{1 / 2}\left[\Psi\left(X_{n}\right)-\Psi(\theta)\right]$ é assintoticamente equivalente a $\Psi^{\prime}(\theta) \cdot n^{1 / 2}\left(X_{n}-\right.$ $\theta)$. Se $X_{n}$ é uma estatística infinito dimensional, como por exemplo uma função de distribuição empírica, é necessário formular o método delta para funcionais, o que requer uma definição mais cuidadosa da derivada.

Sejam $\mathbb{V}, \mathbb{W}$ espaços de funções (em particular, espaços normados equipados com a norma uniforme). Considere $t \in \mathbb{R}$ e $\mathcal{L}$ uma coleção de subconjuntos de $\mathbb{V}$. Suponha um funcional $\Psi: \mathbb{V} \rightarrow \mathbb{W}$. Então, para $F \in \mathcal{S} \subset \mathcal{L}$ : $\Psi$ é $\mathcal{L}$-diferenciável em $F$ com derirada $\Psi_{F}^{\prime}$ se

$$
\lim _{t \rightarrow 0} \frac{\Psi(F+t H)-\Psi(F)}{t}-\Psi_{F}^{\prime}(H)=0 .
$$


uniformemente para qualquer $H \in \mathcal{S}$.

Existem três tipos particulares de diferenciação:

- Se $\mathcal{L}=\{$ todos os singletons de $\mathbb{V}\}, \Psi$ é dita direcionalmente diferenciável ou Gateaux diferenciável,

- se $\mathcal{L}=\{$ todos os subconjuntos compactos de $\mathbb{V}\}$, $\Psi$ é dita compactamente diferenciável ou Hadamard diferenciável,

- se $\mathcal{L}=\{$ todos os subconjuntos limitados de $\mathbb{V}\}$, $\Psi$ é dita limitadamente diferenciável ou Fréchet diferenciável.

Note que diferenciabilidade limitada implica diferenciabilidade compacta e esta implica diferenciabilidade direcional.

A diferenciabilidade de Gateaux é chamada diferenciabilidade direcional pois, para cada possível direção $H$ no dominio, $\Psi_{F}^{\prime}(H)$ mede a direção de uma mudança infinitesimal no valor da função $\Psi$. Em aplicações estatísticas, diferenciabilidade de Gateaux pode ser um conceito fraco.

Uma aplicação $\Psi: \mathbb{V}_{\Psi} \rightarrow \mathbb{W}$, definida em um subconjunto $\mathbb{V}_{\Psi}$ de um espaço normado $\mathbb{V}$ que contém $F$ é chamada Hadamard diferenciável em $F$ se existe uma aplicação linear e contínua $\Psi_{F}^{\prime}: \mathbb{V} \rightarrow \mathbb{W}$ tal que

$$
\left\|\frac{\Psi\left(F+t H_{t}\right)-\Psi(F)}{t}-\Psi_{F}^{\prime}(H)\right\| \rightarrow 0 .
$$

quando $t \rightarrow 0$ e $H_{t} \rightarrow H$ (mais precisamente. para todo $H_{t} \rightarrow H$ tal que $F+t H_{t} \in \mathbb{V}_{\Psi}$. para todo $\left.t>0\right)$.

Note que para a diferenciabilidade de Harlamard as direçōes $H_{t}$ são possíveis 
de mudar com $t$ enquanto que para a diferenciabilidade de Gateaux elas são fixas.

No caso de $\Psi_{F}^{\prime}$ existir em um subconjunto $\mathbb{V}_{0}$ e a sequência $H_{t} \rightarrow H$ ser restrita a convergir a limite $H \in \mathbb{V}_{0}, \Psi$ é chamada de Hadamard diferenciável tangencialmente a este subconjunto.

Diferenciabilidade de Hadamard é chamada também diferenciabilidade compacta descle que se tenha (A.1) uniformemente para $H \subset \mathbb{V}$.

Uma forma mais forte de diferenciabilidade é a de Fréchet. Uma aplicação $\Psi: \mathbb{V}_{\Psi} \rightarrow \mathbb{W}$ é chamada Fréchet diferenciável em $F$ se existir uma aplicação linear e contínua $\Psi_{F}^{\prime}: \mathbb{V} \rightarrow \mathbb{W}$ tal que

$$
\left\|\Psi(F+H)-\Psi(F)-\Psi_{F}^{\prime}(H)\right\|=o(\|H\|)
$$

quando $\|H\| \rightarrow 0$.

Dado que sequências do tipo $t H_{t}$, como as usadas na definição de diferenciabilidade de Hadamard, tem normas que satisfazem $\left\|t H_{t}\right\|=O(t)$, diferenciabilidade de Fréchet é o mais restritiva dos très conceitos. Em aplicações estatísticas, diferenciabilidade de Fréchet pode não ocorrer mas diferenciabilidade de Hadamard sim.

No seguinte teorema estabelecemos o Método Delta para funcionais.

\section{Teorema A.1}

Sejam $\mathbb{V}$ e $\mathbb{W}$ espaços lineares normados. Seja $\Psi: \nabla_{\Psi} \subset \mathbb{V} \rightarrow \mathbb{W}$ Hadamard diferenciável em $F$ tangencialmente a $\mathbb{V}_{0}$. Sejam $Y_{n}: \Omega_{n} \rightarrow \mathbb{V}_{\Psi}$ aplicações tal que $r_{n}\left(X_{n}-F\right) \stackrel{D}{\longrightarrow} Z$ para alguma sequência de números 
$r_{n} \rightarrow \infty$ e um elemento aleatório $Z$ que toma valores em $\mathbb{V}_{0}$. Então $r_{n}\left[\Psi\left(X_{n}\right)-\Psi(F)\right] \stackrel{D}{\longrightarrow} \Psi_{F}^{\prime}(Z)$. Se $\Psi_{F}^{\prime}(Z)$ é definida e contínua no espaço $\mathbb{V}$; então temos também $r_{n}\left[\Psi\left(X_{n}\right)-\Psi(F)\right]=\Psi_{F}^{\prime}\left[r_{n}\left(X_{n}-F\right)\right]+o_{p}(1)$.

Uma propriedade importante da diferenciabilidade de Hadamard é que ela satisfaz a regra da cadeia. Considere as aplicaçōes $\Psi: \mathbb{V} \rightarrow \mathbb{W}$ e $\Phi: \mathbb{W} \rightarrow \mathbb{X}$ sendo Hadamard diferenciaveis em $F$ e $\Phi(F)$, respectivamente. Então a aplicação composição $\Phi \circ \Psi: \mathbb{V} \rightarrow \mathbb{X}$ é Hadamard diferenciável em $F$; e a derivada é a aplicação obtida pela composição das derivadas das duas aplicações. A vantagem da regra da cadeia é que ela permite o cálculo de aplicações Hadamard diferenciáveis, em que a diferenciabilidade de uma aplicação complexa pode ser estabelecida decompondo esta em uma sequência de aplicações mais simples, em que diferenciabilidade de Hadamard seja conhecida ou possa ser provada de um jeito menos complicado.

O seguinte teorema estabelece a regra da cadeia.

\section{Teorema A.2}

Sejam $\Psi: \mathbb{V}_{\Psi} \rightarrow \mathbb{W}_{\Phi}$ e $\Phi: \mathbb{W}_{\Phi} \rightarrow \mathbb{X}$ aplicações definidas em subconjuntos $\mathbb{V}_{\Psi}$ e $\mathbb{W}_{\Phi}$ de espaços normados $\mathbb{V}$ e $\mathbb{W}$, respectivamente. Seja $\Psi$ Hadamard diferenciável em $F$ tangencialmente a $\mathbb{V}_{0}$ e seja $\Phi$ Hadamard diferenciável em $\Psi(F)$ tangencialmente a $\Psi_{F}^{\prime}\left(\mathbb{V}_{0}\right)$. Então $\Phi \circ \Psi: \mathbb{V}_{\Psi} \rightarrow \mathbb{X}$ é Hadamard diferenciável em $F$ tangencialmente a $\mathbb{V}_{0}$ com derivada $\Phi_{\Psi(F)}^{\prime} \circ \Psi_{F}^{\prime}$.

Considere agora $F_{n}$ sendo a função cle distribuição empírica associada a una amostra $x_{1} \ldots \ldots x_{n}$ de uma certa função de distribuição $F$. Dado 
que $F_{n}$ é um estimador fortemente consistente de $F$, i.e., $\left\|F_{n}-F\right\| \stackrel{\text { q.c. }}{\longrightarrow} 0$ quando $n \rightarrow \infty$, desejamos estudar o comportamento de $\Psi\left(F_{n}\right)-\Psi(F)$, com $\Psi: \mathbb{V} \mapsto \mathbb{R}$.

Então, considerando uma expansão de von Mises para o funcional $\Psi$ avaliado em $F_{n}$, em torno de $F$ : podemos escrever;

$$
\Psi\left(F_{n}\right)-\Psi(F)=\Psi_{F}^{\prime}\left(F_{n}-F\right)+\operatorname{Res}\left(F_{n}-F ; \Psi\right)
$$

em que $\operatorname{Res}(\cdot, \Psi)$ corresponde ao resto da aproximação da expansão de primeiro ordem. Quando $\Psi_{F}^{\prime}$ é a derivada de Hadamard é possível mostrar que

$$
n^{1 / 2} \operatorname{Res}\left(F_{n}-F ; \Psi\right) \stackrel{P}{\longrightarrow} 0 ; \quad n \rightarrow \infty
$$

Em particular, o termo $\Psi_{F}^{\prime}\left(F_{n}-F\right)=n^{-1} \sum_{i=1}^{n} \mathrm{IC}\left(X_{i} ; F, \Psi\right)$ é linear e portanto é uma soma de variáveis aleatórias independentes e identicamente distribuídas, com $\mathrm{IC}\left(x_{i} ; F, \Psi\right)$, chamada curva de influência de $\Psi$ em $F$, definida por

$$
\operatorname{IC}\left(X_{i} ; F, \Psi\right)=\frac{d}{d t} \Psi\left(F+t\left(F_{n}-F\right)\right)_{\mid t=0} .
$$

O seguinte teorema estabelece o Método Delta funcional quando o interesse é estudar algum funcional da função de distribuição empírica e faz a ligação com estatística robusta (veja por exemplo, Hampel et al. (1986) e Jurečková e Sen (1996)), através da curva de influência. 


\section{Teorema A.3}

Suponha $\Psi: D[-\infty, \infty] \mapsto \mathbb{R}$ Hadamard diferenciável em $F$ e suponha $\Psi\left(F_{n}\right)$ uma variável aleatória em que $F_{n}$ é a função de distribuição empírica baseada em $n$ observações $X_{1}, \ldots, X_{n}$ independentes e identicamente distribuídas, de uma distribuição $F$ em $\mathbb{R}$. Então, assumindo $0<\sigma^{2}=\operatorname{Var}\left[\operatorname{IC}\left(X_{i} ; F, \Psi\right)\right]<$ $\infty$,

$$
n^{1 / 2}\left[\Psi\left(F_{n}\right)-\Psi(F)\right]=n^{1 / 2} \Psi_{F}^{\prime}\left(F_{n}-F\right) \stackrel{D}{\longrightarrow} N\left(0, \sigma^{2}\right) .
$$

Para maiores detalhes dos tópicos tratados neste apêndice algumas referências são Fernholz (1983), Gill (1989), van der Vaart (1998) e van der Vaart e Wellner (1996). Um enfoque heurístico é dado em Lehmann (1999), Capítulo 6. Extensões para funcionais definidos em $D[0,1]^{p}$ são estudados em Ren e Sen (1995). Diferenciabilidade de Hadamard de segunda ordem, i.e., quando a derivada de Hadamard de primeira ordem é zero, é pesquisada em Ren e Sen (2001). 


\section{Apêndice B}

\section{Alguns conceitos e definições}

A seguir, repassamos alguns conceitos, definições e teoremas utilizados neste trabalho. Referências a estes tópicos podem ser encontradas, por exemplo, em Kolmogorov e Fomin (1972) e Lima (2003) e (2004).

Teorema B.1

Para que $f: X \rightarrow \mathbb{R}$ seja contínua no ponto $a \in X$ é necessário e suficiente que se tenha $\lim f\left(x_{n}\right)=f(a)$ para toda seqüência de pontos $x_{n} \in X$ com $\lim x_{n}=a$.

\section{Teorema B.2}

Se $f: g: X \rightarrow \mathbb{R}$ são contínuas no ponto $a \in X$ então $f+g: f-g$ e $f \cdot g$ são contínuas nesse mesmo ponto. Se $g(a) \neq 0$. então $f / g$ também é contínua no ponto $a$. 


\section{Teorema B.3}

A composta de duas funções contínuas é contínua. Ou seja, se $f: X \rightarrow \mathbb{R}$ e $g: Y \rightarrow \mathbb{R}$ são contínuas nos pontos $a \in X, b=f(a) \in Y$, respectivamente, e, além disso, $f(X) \subset Y$, então $g \circ f: X \rightarrow \mathbb{R}$ é contínua no ponto $a$.

\section{Teorema B.4}

Seja $f: X \rightarrow \mathbb{R}$ contínua. Se $X$ é compacto então $f(X)$ é compacto.

\section{Corolário B.1}

Toda função contínua $f: X \rightarrow \mathbb{R}$ definida em um compacto $X$ é limitada e atinge seus extremos.

\section{Eqüicontinuidade}

\section{Definição B.1}

Seja $E$ um conjunto de funções $f: X \rightarrow \mathbb{R}$; todas com o mesmo domínio $X \subset \mathbb{R}$. Dado $x_{0} \in \mathbb{R}$, dizemos que o conjunto $E$ é eqüicontínuo no ponto $x_{0}$ quando, dado arbitrariamente $\varepsilon>0$, existir $\delta>0$ tal que

$$
x \in X .\left|x-x_{0}\right|<\delta \Rightarrow\left|f(x)-f\left(x_{0}\right)\right|<\varepsilon \text { qualquer que seja } f \in E .
$$




\section{Continuidade uniforme}

\section{Definição B.2}

Uma função $f: X \rightarrow \mathbb{R}$ diz-se uniformemente contínua quando, para cada $\varepsilon>0$, existe $\delta>0$ tal que $x, y \in X, d(x, y)<\delta \Rightarrow d(f(x), f(y))<\varepsilon$.

\section{Teorema B.5}

Seja $X$ compacto. Toda função contínua $f: X \rightarrow \mathbb{R}$ é uniformemente contínua.

\section{Definição B.3}

Sejam $M, N$ espaços métricos. Uma aplicação $f: M \rightarrow N$ diz-se uniformemente contínua quando, para todo $\varepsilon>0$ dado, existir $\delta>0$ tal que sejam quais forem $x, y \in M, d(x, y)<\delta \Rightarrow d(f(x), f(y))<\varepsilon$.

\section{Teorema B.6}

Se o espaço métrico $M$ é compacto, então toda aplicação contínua $f: M \rightarrow N$ é uniformemente contínua. 


\section{Referências Bibliográficas}

Andersen, P.K., Borgan, Ø., Gill, R.D. e Keiding, N. (1993). Statistical Models Based on Counting Processes, Springer-Verlag, New York, Inc.

Anderson, J.E., Louis, T.A., Holm, N.V. e Harvald, B. (1992). Timedependent association measures for bivariate survival distributions. Journal of the American Statistical Association, 87, 641-650.

Chen, M.H. e Schmeiser, B. (1993). Performance of the Gibbs, hit-and-run and Metropolis samplers. Journal of the Computational and Graphical Statistics, 2, 251-272.

Clayton, D.G. (1978). A model for association in bivariate life-tables and its application in epidemiological studies of familial tendency in chronic disease incidence. Biometrika, 65, 141-151.

Cox, D.R. (1972). Regression models and life-tables (with discussion). Journal of the Royal Statistical Society B, 34, 187-220.

Dabrowska, D.M. (1989). Kaplan-Meier estimate on the plane: Weak convergence, LIL, and the Bootstrap. Journal of Multivariate Analysis. 29, 308-325. 
Efron, B.F. (1982). The Jackknife: the Bootstrap, and Other Resampling Plans, Philadelphia: Society for Industrial and Applied Mathematics.

Embrechts, P., Linskog, F. e McNiel, A. (2003). Modelling dependence with copulas and applications to risks management. http://www.math.ethz.ch/ baltes/ftp/papers.html.

Embrechts, P., McNiel, A. e Straumann, D. (2002). Correlation and dependence in risk management: properties and pitfalls. http://www.math.ethz.ch/ baltes/ftp/papers.html.

Fan, J., Prentice, R.L. e Hsu, L. (2000). A class of weighted dependence measures for bivariate failure time data. Journal of the Royal Statistical Society $B, 62,181-190$.

Fang, K.-T., Kotz, S. e Ng, K.W. (1987). Symmetric Multivariate and Related Distributions, Chapman \& Hall, London.

Feller, W. (1971). An Introduction to Probability Theory and its Applications, Volume 2, 2nd Edition, New York: Wiley.

Fernholz, L.T. (1983). Von Mises Calculus for Statistical Functional. Lecture Notes in Statistics, Vol. 19, Springer-Verlag; New York.

Frank: M.J. (1979). On the simultaneous associativity of $F(x, y)$ and $x+$ $y-F(x, y)$. Aequations Mathematicae, 19, 194-226.

Freireich, E.J., Gehan, E.A., Frei, E., Schroeder, L.R., Wolman, I.J., Anbari, R.. Burgert, E.O., Mills, S.D., Pinkel, D., Selawry: O.S., Moon. J.H.. Gendel. B.R.. Spurr, C.L., Storrs, R., Haurani. F.. Hoogstraten. B. e Lee S. (196:3). The effect of 6-Mercaptopurine on the duration of 
steroid-induced remissions in acute leukemia: a model for evaluation of other potentially useful therapy. Blood, 21, 699-716.

Gelfand, A.E. (1996). Model determination using sampling based methods. In Markov Chain Monte Carlo in Practice (Eds. W.R. Gilks, S. Richardson and D.J. Spiegelhalter), Chapman and Hall: London, $145-161$.

Gelfand, A.E. e Ghosh, S.K. (1998). Model choice: A minimum posterior predictive loss approach. Biometrika, 85, 1, 1-11.

Genest, C. (1987). Frank's family of bivariate distributions. Biometrika, $74,3,549-555$.

Genest, C., Ghoudi, K. e Rivest, L.P. (1995). A semiparametric estimation procedure of dependence parameters in multivariate families of distributions. Biometrika, 82, 3, 543-552.

Genest, C. e MacKay, J. (1986). The joy of copulas: bivariate distribution with uniform marginals. The American Statistician, 40, 280-283.

Geweke, J. (1992). Evaluating the accuracy of sampling-based approaches to the calculation of posteriori moments. In Bayesian Statistics 4 (Eds. J.M. Bernardo, J.O. Berger, A.P. Dawid and A.F.M. Smith); Oxford: Oxford University Press, 169-193.

Geyer, C. (1992). Practical Markov Chain Monte Carlo. Statistical Science, 7. $473-482$.

Gilks, W.R. e Wild, P. (1992). Adaptive rejection sampling for Gibbs sampling. Applied Statistics, 41, 2, 337-348. 
Gill, R.D. (1989). Non- and semi-parametric maximum likelihood estimators and the von Mises method (Part 1). Scandinavian Journal of Statistics, 16, 97-128.

Gustafson, P., Aeschliman, D. e Levy, A.R. (2003). A simple approach to fitting Bayesian survival models. Lifetime Data Analysis, 9, 5-19.

Hampel, F.R., Ronchetti, E.M., Rousseuw, P.J. e Stahel, W.A. (1986). Robust Statistics: The Approach based on Influence Functions, Wiley, New York.

Hastings; W.K. (1970). Monte Carlo sampling methods using Markov chains and their applications. Biometrika, 57, 97-109.

Hjört, N.L. (1990). Non-parametric Bayes estimators based on Beta processes in models for life history data. The Annals of Statistics, 18, $1259-1294$.

Hougaard, P. (1986a). Survival models for heterogeneous populations derived from stable distributions. Biometrika, 73, 387-396.

Hougaard, P. (1986b). A class of multivariate failure time distributions. Biometrika, 73, 671-678.

Hougaard, P. (1989). Fitting a multivariate failure time distribution. IEEE Transactions on Reliability, 38, 444-448.

Hougaard. P. (2000). Analysis of Multivariate Survival Data, Springer, New York.

Huster. IV.J.. Brookmeyer, R. e Self. S.G. (1989). Modeling paired survival data with covariates. Biometrics. 45. 145-156. 
Ibrahim, J.G., Chen, M-H., e Sinha, D. (2001). Bayesian Survival Analysis, Springer Verlag, New York.

James, B.R. (2004). Probabilidade: Um Curso em Nível Intermediário, Terceira edição, Coleção Projeto Euclides, CNPq. Rio de Janeiro: IMPA.

Joe, H. (1997). Multivariate Models and Dependence Concepts, Chapman \& Hall, London.

Jurečková, J. e Sen, P.K. (1996). Robust Statistical Procedures, Wiley, New York.

Kolmogorov, A.N. e Fomin, S.V. (1972). Elementos de la Teoría de Funciones y del Análisis Funcional, Editorial Mir, Moscú.

Lee, L. (1979). Multivariate distributions having Weibull properties. Journal of Multivariate Analysis, 9, 267-277.

Lehmann, E.L. (1999). Elements of Large-Sample Theory, Springer-Verlag, New York, Inc.

Lima, E.L. (2003). Espaços Métricos, Terceira edição, Coleção Projeto Euclides, CNPq. Rio de Janeiro: IMPA.

Lima, E.L. (2004). Curso de Análise, Volume 1, Décima primeira edição, Coleção Projeto Euclides, CNPq. Rio de Janeiro: IMPA.

Maller, R.A. e Zhou, X. (1996). Survival Analysis with Long-Term Survivors, John Wiley and Sons Ltd, Chichester.

Marshall. A.W. e Olkin, I. (1988). Families of multivariate distributions. Journal of the American Statistical Association. 83. 834-841. 
McLeish, D.L. e Small, C.G. (1988). The Theory and Applications of Statistical Inference Functions. Lecture Notes in Statistics, 44. SpringerVerlag, New York.

Metropolis, N., Rosenbluth, A.W., Rosenbluth, M.N., Teller, A.H. e Teller, E. (1953). Equations of state calculations by fast computing machines. Journal of Chemical Physics, 21, 1087-1092.

Neal, R. (2003). Slice sampling. The Annals of Statistics, 31, 3, 705-767.

Nelsen, R. (1986). Properties of a one-parametric family of bivariate distributions with specified marginals. Communications in Statistics Theory and Methods, 15, 3277-3285.

Nelsen, R. (1999). An Introduction to Copulas, Springer, New York.

Oakes, D. (1989). Bivariate survival models induced by frailties. Journal of the American Statistical Association, 84, 487-493.

Owzar, K. e Sen, P.K. (2003). Copulas: concepts and novel applications. METRON, LXI, n. 3, 323-353.

Ren, J.J. e Sen, P.K. (1991). On Hadamard differentiability of extended statistical functional. Journal of Multivariate Analysis, 39, 30-43.

Ren, J.J. e Sen, P.K. (1995). Hadamard differentiability on $D[0,1]^{P}$. Journal of Multivariate Analysis, 55, 14-28.

Ren, J.J. e Sen, P.K. (2001). Second order Hadamard differentiability in statistical applications. Journal of Multivariate Analysis, 77. 187-228.

Sahu. S.K. e Dey. D.K. (2000). A comparison of frailty and other models for bivariate survival data. Lifetime Data Analysis: 6. 207-228. 
Salinas-Torres, V.H. (1992). Análise Bayesiana não-paramétrica na teoria de riscos competitivos. Tese de Doutorado, IME/USP.

Salinas-Torres, V.H., Pereira, C.A.B. e Tiwari, R.C. (2002). Bayesian nonparametric estimation in a competing risks model or a series system. Journal of Nonparametric Statistics, 14, 449-458.

Schweizer, B. e Sklar, A. (1983). Probabilistic Metric Spaces, NorthHolland/ Elsevier, New York.

Shih, J.H. e Louis, T.A. (1995). Inferences on the association parameter in copula models for bivariate survival data. Biometrics, 51, 1384-1399.

Sklar, A. (1959). Fonctions de répartition à $n$ dimensions et leurs marges. Publ. Inst. Statist. Univ. Paris, 8, 229-231.

Sklar, A. (1996). Random variables, distribution functions, and copulas - a personal look backward and forward. In Distribution with Fixed Marginals and Related Topics, (Eds. L. Rüschendorff, B. Schweizer and M. Taylor), Institute of Mathematical Statistics, Hayward, CA, $1-14$.

Smith, B.J. (2005) Bayesian Output Analysis Program, BOA 1.1.5 for R. Disponível em http://www.public-health.uiowa.edu/boa.

Spiegelhalter, D.J., Thomas, A. e Best, N.G. (2003). WinBugs 1.4.1 "Computer Program". Imperial College \& MRC Biostatistics Unit, IPH. Cambridge, UK. Disponível em http://www.mrc-bsu.cam.ac.uk/bugs.

Susarla, V. e Van Ryzin, J. (1976). Non-parametric Bayesian estimation of survival curves from incomplete observations. Journal of the American Statistical Association, 71, 897-902. 
Tanner, M.A. (1996). Tools for Statistical Inference: Methods for the Exploration of Posterior Distributions and Likelihood Functions, Third edition, Springer-Verlag: New York.

The Diabetic Retinopathy Study Research Group (1976). Preliminary report on the effect of photocoagulation therapy. American Journal of Ophthalmology, 81, 383-396.

Therneau, T.M. e Grambsch, P. (2000). Modeling Survival Data: Extending the Cox Model, Springer, New York.

van der Vaart, A.W. (1998). Asymptotic Statistics, Cambridge University Press.

van der Vaart, A.W. e Wellner, J.A. (1996). Weak Convergence and Empirical Processes, Springer, New York.

Vaupel, J.W., Manton, K.G. e Stallard, E. (1979). The impact of heterogeneity in individual frailty on the dynamics of mortality. Demography, $16,439-454$.

von Mises, R. (1947). On the asymptotic distribution of differentiable statistical functions. Annals of Mathematical Statistics, 18, 309-348.

Wang, W. e Wells, M.T. (2000). Model selection and semiparametric inference for bivariate failure-time data. Journal of the American Statistical Association, 95, 62-72. 